\title{
The Index of Dirac Operators on Incomplete Edge Spaces
}

\author{
Pierre $A L B I N^{\dagger}$ and Jesse GELL-REDMAN $\ddagger$ \\ $\dagger$ University of Illinois, Urbana-Champaign, USA \\ E-mail: palbin@illinois.edu \\ URL: http://www.math.uiuc.edu/ palbin/ \\ $\ddagger$ Department of Mathematics, University of Melbourne, Melbourne, Australia \\ E-mail: j.gell@unimelb.edu.au \\ URL: http://www.math.jhu.edu/ jgell/
}

Received November 02, 2015, in final form August 30, 2016; Published online September 08, 2016

http://dx.doi.org/10.3842/SIGMA.2016.089

\begin{abstract}
We derive a formula for the index of a Dirac operator on a compact, evendimensional incomplete edge space satisfying a "geometric Witt condition". We accomplish this by cutting off to a smooth manifold with boundary, applying the Atiyah-Patodi-Singer index theorem, and taking a limit. We deduce corollaries related to the existence of positive scalar curvature metrics on incomplete edge spaces.
\end{abstract}

Key words: Atiyah-Singer index theorem; Dirac operators; singular spaces; positive scalar curvature

2010 Mathematics Subject Classification: 58G10; 58A35; 58G05

\section{Contents}

1 Introduction $\quad 2$

2 Connection and Dirac operator $\quad \mathbf{7}$

2.1 Incomplete edge metrics and their connections . . . . . . . . . . . . . . . . . 7

2.2 Clifford bundles and Clifford actions . . . . . . . . . . . . . . . . . . . 12

2.3 The APS boundary projection . . . . . . . . . . . . . . . . . . . . . . . . . . . . . . . .

3 Mapping properties of $\partial \quad 14$

3.1 The "geometric Witt condition" . . . . . . . . . . . . . . . . . . . . . . . 14

3.2 Review of edge and incomplete edge operators . . . . . . . . . . . . . . . . . . . 15

3.3 Parametrix of $x \partial$ on weighted edge spaces . . . . . . . . . . . . . . . . . . . . 15

3.4 Proof of Theorem 1.1 and the generalized inverse of $\delta$. . . . . . . . . . . . . . 23

4 Boundary values and boundary value projectors 24

4.1 Boundary value projector for $\mathcal{D}_{\varepsilon} \ldots \ldots \ldots$. . . . . . . . . . . . . . . . . . . . 25

5 Equivalence of indices 29

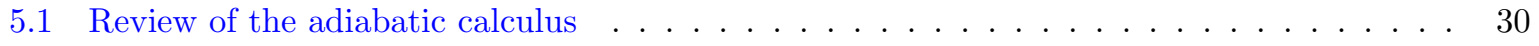

5.2 APS projections as an adiabatic family . . . . . . . . . . . . . . . . . . 32

6 Proof of Main Theorem: limit of the index formula 34

6.1 Four-dimensions with circle fibers . . . . . . . . . . . . . . . . . 36

7 Positive scalar curvature metrics 40

$\begin{array}{ll}\text { A Appendix } & 41\end{array}$

$\begin{array}{ll}\text { References } & 43\end{array}$ 


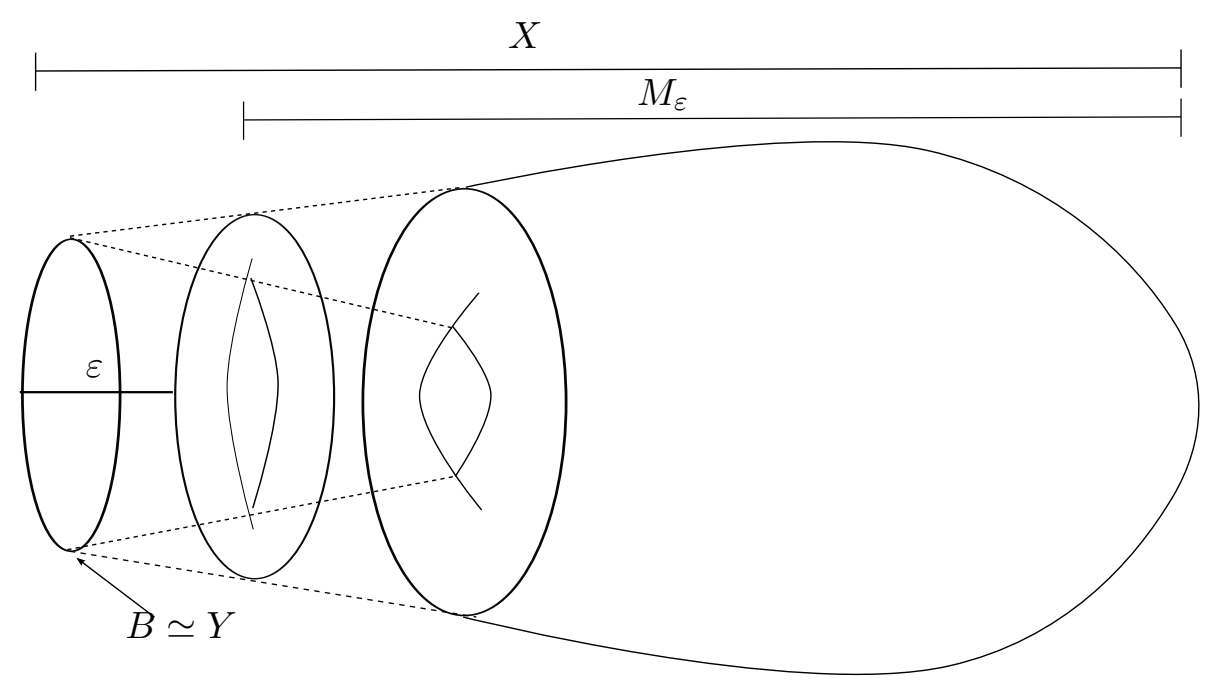

Figure 1. The singular space $X$ obtained by collapsing the fibers of the boundary fibration of $M$. The spaces $M_{\varepsilon}$ play a central role in our proofs.

\section{Introduction}

Ever since Cheeger's celebrated study of the spectral invariants of singular spaces [22, 23, 24] there has been a great deal of research to extend our understanding of geometric analysis from smooth spaces. Index theory in particular has been extended to spaces with isolated conic singularities quite successfully (beyond the papers of Cheeger see, e.g., [20, 31, 32, 43]) and was used by Bismut and Cheeger to establish their families index theorem on manifolds with boundary $[13,14,15]$.

The fact that Bismut and Cheeger used, [13, Theorem 1.5], is that for a Dirac operator on a spin space with a conic singularity, the null space of $L^{2}$ sections naturally corresponds to the null space of the Dirac operator on the manifold with boundary obtained by excising the singularity and imposing the 'Atiyah-Patodi-Singer boundary condition' [8], provided an induced Dirac operator on the link has no kernel. (Here 'Dirac operator' refers to the 'classical' Dirac operator $\operatorname{Tr} c \circ \nabla$ where $\nabla$ is the Levi-Cevita connection on the spin bundle and $c$ is Clifford multiplication.) This is not true for more general first order operators; indeed, even for twisted Dirac operators there is no such equivalence. On the other hand, Cheeger points out in [22] that one can leverage the related APS boundary value problems to prove the Gauss-Bonnet and Hirzebruch signature theorems for spaces with conic singularities.

In this paper we consider the Dirac operator on a spin space with non-isolated conic singularities, also known as an 'incomplete edge space', and the Dirac operator on the manifold with boundary obtained by excising a tubular neighborhood of the singularity and imposing the Atiyah-Patodi-Singer boundary condition. Although the relation between the domains of these two Dirac operators is much more complicated than in the case of isolated conic singularities, we show that under a "geometric Witt assumption" analogous to that used by Bismut-Cheeger, the indices of these operators coincide. Thus we obtain a formula for the index of the Dirac operator on the singular space as the 'adiabatic limit' of the index of the Dirac operator with Atiyah-Patodi-Singer boundary conditions.

An incomplete edge (ie) space is a stratified space $X$ with a single singular stratum $Y$. In keeping with Melrose's paradigm for analysis on singular spaces (see, e.g., [50, 52]) we resolve $X$ by 'blowing-up' $Y$ and obtain a smooth manifold with boundary $M$, whose boundary is the total space of a fibration of smooth manifolds with typical fiber $Z$,

$$
Z \hookrightarrow \partial M \stackrel{\phi}{\longrightarrow} Y .
$$


A (product-type) incomplete edge metric is a metric that, in a collar neighborhood of the boundary, takes the form

$$
g=d x^{2}+x^{2} g_{Z}+\phi^{*} g_{Y}
$$

with $x$ a defining function for $\partial M, g_{Y}$ a metric on $Y$ and $g_{Z}$ a family of two-tensors that restrict to a metric on each fiber of $\phi$. Thus we see that metrically the fibers of the boundary fibration are collapsed, as they are in $X$.

We also replace the cotangent bundle of $M$ by a bundle adapted to the geometry, the 'incomplete edge cotangent bundle' $T_{\mathrm{ie}}^{*} M$, see (2.2) below. This bundle is locally spanned by forms like $d x, x d z$, and $d y$, and the main difference with the usual cotangent bundle is that the form $x d z$ is a non-vanishing section of $T_{\mathrm{ie}}^{*} M$ all the way to $\partial M$.

We assume that $M^{\circ}$ is spin and denote a spin bundle on $M^{\circ}$ by $\mathcal{S} \longrightarrow M$ and the associated Dirac operator by $\precsim$. The operator $\precsim$ does not induce an operator on the boundary in the usual sense, due to the degeneracy of the metric there, but we do have

$$
\left.x ð\right|_{\partial M}=c(d x)\left(\frac{1}{2} \operatorname{dim} Z+\partial_{Z}\right),
$$

where $\partial_{Z}$ is a family of operators on the fibers of $\partial M$. It turns out, as in the conic case mentioned above, and the analogous study of the signature operator in $[2,3]$, that much of the functional analytic behavior of $\precsim$ is tied to that of $\partial_{Z}$. Indeed, in Section 3.4 below we prove the following theorem.

Theorem 1.1. Assume that $\partial$ is a Dirac operator on a compact, spin incomplete edge space $(M, g)$, satisfying the "geometric Witt-assumption"

$$
\operatorname{Spec}\left(\check{\partial}_{Z}\right) \cap(-1 / 2,1 / 2)=\varnothing .
$$

Then the unbounded operator $\precsim$ on $L^{2}(M ; \mathcal{S})$ with core domain $C_{c}^{\infty}(M ; \mathcal{S})$ (sections supported in $M \backslash \partial M)$ is essentially self-adjoint. Moreover, letting $\mathcal{D}$ denote the domain of this self-adjoint extension, the map

$$
\text { ə: } \mathcal{D} \longrightarrow L^{2}(M ; \mathcal{S})
$$

is Fredholm.

When $M$ is even-dimensional, the spin bundle admits the standard $\mathbb{Z} / 2 \mathbb{Z}$ grading into even and odd spinors

$$
\mathcal{S}=\mathcal{S}^{+} \oplus \mathcal{S}^{-}
$$

and thus we have the chirality spaces $\mathcal{D}^{ \pm}=\mathcal{D} \cap L^{2}\left(M ; \mathcal{S}^{ \pm}\right)$and the restriction of the Dirac operator satisfies

$$
\text { ə: } \mathcal{D}^{+} \longrightarrow L^{2}\left(M ; \mathcal{S}^{-}\right) .
$$

This map is Fredholm and our main result is an explicit formula for its index.

The metric (1.1) naturally defines a bundle metric on $T_{\mathrm{ie}}^{*} M$, non-degenerate at $\partial M$, and the Levi-Civita connection of $g$ naturally defines a connection $\nabla$ on $T_{\mathrm{ie}}^{*} M$. Our index formula involves the transgression of a characteristic class between two related connections. The restriction of $T_{\mathrm{ie}} M$ to $\partial M$ can be identified with $N_{M} \partial M \oplus T \partial M / Y \oplus \phi^{*} T Y$. Let

$$
\mathbf{n}:\left.T_{\mathrm{ie}} M\right|_{\partial M} \longrightarrow N_{M} \partial M, \quad \mathbf{v}:\left.T_{\mathrm{ie}} M\right|_{\partial M} \longrightarrow T \partial M / Y
$$

be the orthogonal projections onto the normal bundle of $\partial M$ in $M, N_{M} \partial M=\left\langle\partial_{x}\right\rangle$, and the vertical bundle of $\phi$, respectively, and let $\mathbf{v}_{+}=\mathbf{n} \oplus \mathbf{v}$. Both

$$
\nabla^{v_{+}}=\left.\mathbf{v}_{+} \circ \nabla\right|_{\partial M} \circ \mathbf{v}_{+}, \quad \text { and } \quad \nabla^{\mathrm{pt}}=\left.\mathbf{n} \circ \nabla\right|_{\partial M} \circ \mathbf{n} \oplus \mathbf{v} \circ j_{0}^{*} \nabla \circ \mathbf{v}
$$

where pt stands for 'product', are connections on $N_{M} \partial M \oplus T \partial M / Y \longrightarrow \partial M$. 
Main Theorem. Let $X$ be a compact, even-dimensional stratified space with a single singular stratum endowed with an incomplete edge metric $g$ and let $M$ be its resolution. If $\precsim$ is a Dirac operator associated to a spin bundle $\mathcal{S} \longrightarrow M$ and ð satisfies the geometric Witt condition (1.2), then

$$
\operatorname{Ind}\left(\text { ə: } \mathcal{D}^{+} \longrightarrow L^{2}\left(M ; \mathcal{S}^{-}\right)\right)=\int_{M} \widehat{A}(M)+\int_{Y} \widehat{A}(Y)\left(-\frac{1}{2} \widehat{\eta}\left(\mathrm{\partial}_{Z}\right)+\int_{Z} T \widehat{A}\left(\nabla^{v_{+}}, \nabla^{\mathrm{pt}}\right)\right),
$$

where $\widehat{A}$ denotes the $\widehat{A}$-genus, $T \widehat{A}\left(\nabla^{v_{+}}, \nabla^{\mathrm{pt}}\right)$ denotes the transgression form of the $\widehat{A}$ genus associated to the connections (1.3), and $\widehat{\eta}$ the $\eta$-form of Bismut-Cheeger [12].

The simplest setting of incomplete edge spaces occurs when $Z$ is a sphere, as then $X$ can be given a smooth structure and the singularity at $Y$ is entirely in the metric. Atiyah and LeBrun have recently studied the case where $Z=\mathbb{S}^{1}$ and $X$ is four-dimensional, so that $Y$ is an embedded surface, and the metric $g$ asymptotically has the form

$$
d x^{2}+x^{2} \beta^{2} d \theta^{2}+\phi^{*} g_{Y} .
$$

The cone angle $2 \pi \beta$ is assumed to be constant along $Y$. In [7] they find formulas for the signature and the Euler characteristic of $X$ in terms of the curvature of this incomplete edge metric. Kronheimer and Mrowka also study invariants of such spaces, in particular working out the dimension of the moduli space of naturally associated flat connections [39, 40], and Lock and Viaclovsky [44] compute the index of the 'anti-self-dual deformation complex'. Using work of Dai [27] and Dai-Zhang [29], in Theorem 6.2 below, we recover the formula for the signature in [7], and moreover, we show that our formula for the index of the Dirac operator (1.4) simplifies substantially in the case $Z=\mathbb{S}^{1}$ and $\operatorname{dim} X=4$. In the context of general Witt spaces, on the other hand, work on the signature operator and $L$-class in the incomplete edge setting includes [19] and [21].

In studying the Dirac operator on incomplete edges with sphere fibers, it is natural to assume, and we do so below, that the manifold $X$ itself has a spin structure. This induces a spin structure on the interior of $M$ (which is diffeomorphic to $X$ minus the singular locus), which extends to $M$. Then the induced spin structure on the fiber $Z \simeq \mathbb{S}^{f}$ is the spin structure induced from thinking of $\mathbb{S}^{f}$ as the boundary of the ball $\mathbb{B}^{f+1}$ in Euclidean space. In particular when $f=1$, so the fiber $Z$ is a circle, the induced spin structure on $\mathbb{S}^{1}=\mathbb{R} / 2 \pi \mathbb{Z}$ with the round metric $d \theta^{2}$, induce the Dirac operator $\partial_{\theta}=-i \partial_{\theta}+1 / 2$ whose spectrum satisfies $\operatorname{spec}\left(\partial_{\theta}\right)=\{1 / 2+\mathbb{Z}\}$. (Note that the spectrum of the Dirac operator depends on the choice of spin structure, so it is important that we have the bounding spin structure on the circle.) As we show in the proof of the following corollary, this implies that for cone angles $2 \pi \beta \leq 2 \pi$, the geometric Witt assumption is satisfied and we arrive at a simpler index formula in this case.

Corollary 1.2. If $\partial$ is a Dirac operator on a smooth, compact four-dimensional spin manifold $X$, associated to an incomplete edge metric with constant cone angle $2 \pi \beta \leq 2 \pi$ along an embedded surface $Y$, then $\partial$ is essentially self-adjoint and its index is given by

$$
\text { Ind }\left(\text { ð : } \mathcal{D}^{+} \longrightarrow L^{2}\left(M ; \mathcal{S}^{-}\right)\right)=-\frac{1}{24} \int_{M} p_{1}(M)+\frac{1}{24}\left(\beta^{2}-1\right)[Y]^{2},
$$

where $[Y]^{2}$ is the self-intersection number of $Y$ in $X$.

The formulas in (1.4) and (1.5), and indeed our proof, are obtained by taking the limit of the index formula for the Dirac operators on the manifolds with boundary

$$
M_{\varepsilon}=\{x \geq \varepsilon\},
$$


so in particular the contribution from the singular stratum $Y$ is the adiabatic limit $[12,27,62]$ of the $\eta$-invariant from the celebrated classical theorem of Atiyah, Patodi, and Singer [8], which we review in Section 6. It is important to note that the analogous statement for a general twisted Dirac operator is false and the general index formula requires an extra contribution from the singularity. We will return to this in a subsequent publication. We also point out to the reader that there exist other derivations of index formulas on manifolds with structured ends in which the computation is reduced to taking a limit in the Atiyah-Patodi-Singer index formula; see for example [19] where the author discovers a self-adjointness criterion and proves an $L^{2}$-signature theorem on incomplete edge spaces, or [42] where the authors prove an index formula for twisted Dirac operators on spin manifolds with fibered boundary metrics, a complete Riemannian manifold with a structured end that is a fiber bundle over an asymptotically conical (big end of a cone) manifold.

One very interesting aspect of the spin Dirac operator is its close relation to the existence of positive scalar curvature metrics. Most directly, the Lichnerowicz formula shows that the index of the Dirac operator is an obstruction to the existence of such a metric. This is still true among metrics with incomplete edge singularities. Analogously to the results of Chou for conic singularities [25] we prove the following theorem in Section 7.

Theorem 1.3. Let $(M, g)$ be a spin incomplete edge space. The geometric Witt assumption' (1.2) holds if either:

1. $\operatorname{dim} Z \geq 2$ and the scalar curvature of $g$ is non-negative in a neighborhood of $\partial M$.

2. $\operatorname{dim} Z=1$, the spin structure on $M$ is the lift of a spin structure on $X$, and the cone angle satisfies $2 \pi \beta \leq 2 \pi$.

If the geometric Witt assumption holds and in addition the scalar curvature of $g$ is nonnegative on all of $M$, and positive somewhere, then $\operatorname{Ind}(ð)=0$.

Now let us indicate in more detail how these theorems are proved. For convenience we work throughout with a product-type incomplete edge metric as described above, but removing this assumption would only result in slightly more intricate computations below. The proof of Theorem 1.1 follows the arguments employed in $[2,3]$ to prove the analogous result for the signature operator. Thus we start with the two canonical closed extensions of $\mathrm{\partial}$ from $C_{c}^{\infty}(M)$, the sections with support a compact set in the interior $M \backslash \partial M$, namely

$$
\begin{aligned}
& \mathcal{D}_{\text {max }}:=\left\{u \in L^{2}(M ; \mathcal{S}): ð u \in L^{2}(M ; \mathcal{S})\right\}, \\
& \mathcal{D}_{\text {min }}:=\left\{u \in \mathcal{D}_{\text {max }}: \exists u_{k} \in C_{c}^{\infty}(M) \text { with } u_{k} \rightarrow u, \partial u_{k} \rightarrow ð u \text { as } k \rightarrow \infty\right\},
\end{aligned}
$$

where the convergence in the second definition is in $L^{2}(M ; \mathcal{S})$, and we show that under assumption (1.2), these domains coincide

$$
\mathcal{D}_{\text {min }}=\mathcal{D}_{\max }=\mathcal{D} \text {. }
$$

Since $\partial$ is a symmetric operator, this shows that it is essentially self-adjoint.

One difference between the case of isolated conic singularities $(\operatorname{dim} Y=0)$ and the general incomplete edge case is that in the former, even if Assumption (1.2) does not hold, $\mathcal{D}_{\max } / \mathcal{D}_{\min }$ is a finite-dimensional space. In contrast, when $\operatorname{dim} Y>0$, this space is generally infinitedimensional.

We prove (1.7) by constructing a parametrix $\bar{Q}$ for $\partial$ in Section 3. From the mapping properties of $\bar{Q}$, we deduce both that $\partial$ is essentially self-adjoint, and that it is a Fredholm operator from the domain of its unique self-adjoint extension to $L^{2}$. The relationship between the mapping properties of $\bar{Q}$ and the stated conclusions can be seen largely through (3.28) below, 

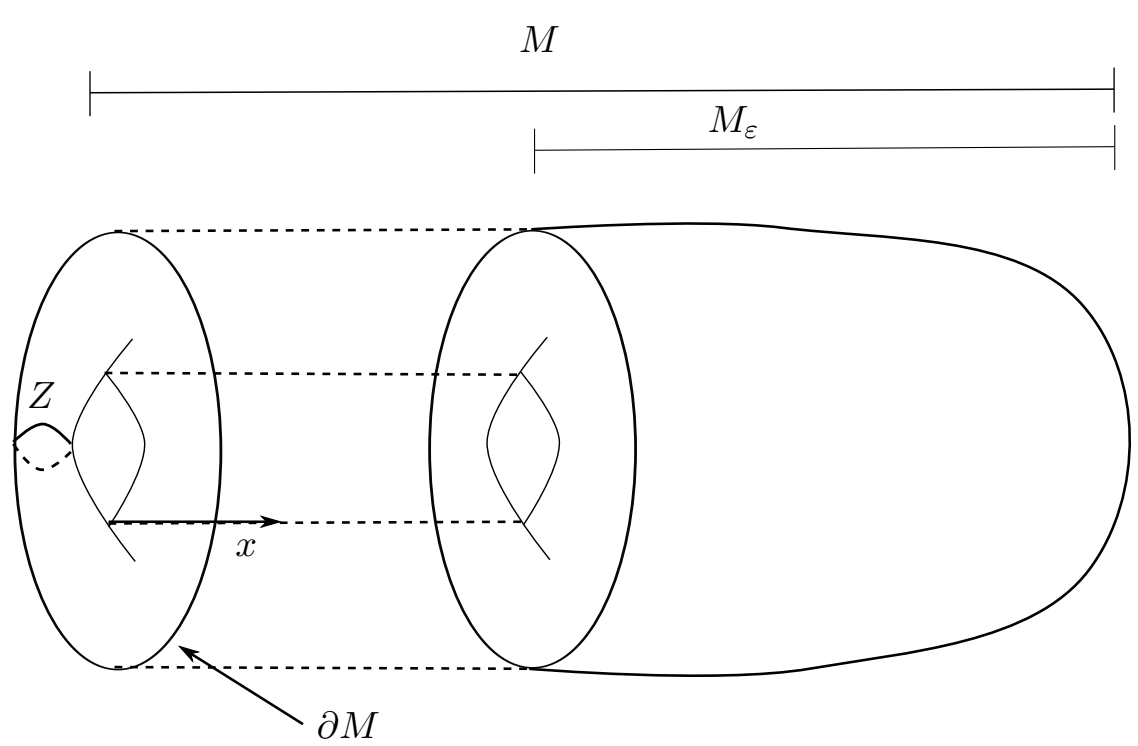

Figure 2. $M$ as a smooth manifold with boundary whose boundary $\partial M$ is a fiber bundle. Here $x$ is a boundary defining function and the space $M_{\varepsilon}$ are given by $M_{\varepsilon}=\{x \geq \varepsilon\}$.

which states that the maximal domain has 'extra' vanishing, i.e., sections in $\mathcal{D}_{\text {max }}$ lie in weighted spaces $x^{\delta} L^{2}$ with weight $\delta$ higher than generically expected. This shows that the inclusion of the domain into $L^{2}$ is a compact operator, which in particular gives that the kernel of $\partial$ on the maximal domain is finite-dimensional.

Once this is established we give a precise description of the Schwartz kernel of the generalized inverse $Q$ of $\partial$ using the technology of $[45,47]$. In Section 4 , we use $Q$ and standard methods from layer potentials to construct a family of pseudodifferential projectors

$$
\mathcal{E}_{\varepsilon} \in \Psi^{0}\left(\partial M_{\varepsilon} ; \mathcal{S}\right)
$$

such that

$$
\left.\check{\partial}\right|_{M_{\varepsilon}} \text { with domain } \mathcal{D}_{\varepsilon}=\left\{u \in H^{1}\left(M_{\varepsilon} ; \mathcal{S}\right):\left(\operatorname{Id}-\mathcal{E}_{\varepsilon}\right)\left(\left.u\right|_{\partial M_{\varepsilon}}\right)=0\right\} .
$$

is Fredholm and has the same index as $(ð, \mathcal{D})$. This domain is constructed so that the boundary values coincide with boundary values of ' $\partial$-harmonic' $L^{2}$-sections over the excised neighborhood of the singularity, $M-M_{\varepsilon}$.

To compute this index, we consider the operators

$$
\left.\check{\partial}\right|_{M_{\varepsilon}} \text { with domain } \mathcal{D}_{\mathrm{APS}, \varepsilon}=\left\{u \in H^{1}\left(M_{\varepsilon} ; \mathcal{S}\right):\left(\operatorname{Id}-\pi_{\mathrm{APS}, \varepsilon}\right)\left(\left.u\right|_{\partial M_{\varepsilon}}\right)=0\right\} .
$$

where $\pi_{\mathrm{APS}, \varepsilon}$, is the projection onto the positive spectrum of $\left.\partial\right|_{\partial M_{\varepsilon}}$. From [8] we know that these are Fredholm operators and

$$
\operatorname{Ind}\left(\check{\partial}, \mathcal{D}_{\mathrm{APS}, \varepsilon}^{+} \longrightarrow L^{2}\left(M ; \mathcal{S}^{-}\right)\right)=\int_{M} \widehat{A}(M)-\frac{1}{2} \eta\left(\varlimsup_{\partial M_{\varepsilon}}\right)+\mathcal{L}_{\varepsilon},
$$

where $\mathcal{L}_{\varepsilon}$ is a local integral over $\partial M_{\varepsilon}$ compensating for the fact that the metric is not of producttype at $\partial M_{\varepsilon}$. These domains depend fundamentally on $\varepsilon$. Not only does $\mathcal{D}_{\mathrm{APS}, \varepsilon}$ vary as $\varepsilon \rightarrow 0$, it does not limit to a fixed subspace of $L^{2}(\partial M)$ with any natural metric. (More precisely, the boundary value projectors $\pi_{\mathrm{APS}, \varepsilon}$ which define the boundary condition do not converge in norm.)

Through a semiclassical analysis, which we carry out using the adiabatic calculus of MazzeoMelrose [46], we show that the projections $\mathcal{E}_{\varepsilon}$ and $\pi_{\mathrm{APS}, \varepsilon}$ are homotopic for small enough $\varepsilon$, 
with a homotopy through operators with the same principal symbol. The adiabatic calculus technology boils this down to an explicit analysis of modified Bessel functions, which we carry out in the appendix. Then we can appeal to arguments from Booss-Bavnbek-Wojciechowski [17] to see that the two boundary value problems have the same index. Having shown that the index of $(ð, \mathcal{D})$ is equal to the adiabatic limit of the index formula (1.8), the Main Theorem follows as shown in Section 6.

Incomplete edge differential operators, such as the Dirac operator for on an incomplete edge spin manifold, are closely related with (complete) edge differential operators, which include the natural geometric PDEs associated with edge metrics. Indeed, our analysis of $\partial$ here relies partly on pre-existing analysis of edge differential operators [48], and in the case of the Dirac operator of a given spin structure (and not in the general case of twisted Dirac operators) the complete and incomplete edge cases are related by conformal invariance properties. As we mention in Remark 3.5, if the geometric Witt assumption is strengthened to exclude fiber spectrum from the closed interval $[-1 / 2,1 / 2]$, then our results imply an index formula for Dirac operators on edge manifolds (which under this strengthened assumption are Fredholm on their natural domain.)

Though we do not attempt to give a complete overview here, there is a large body of work on index theory of non-compact Riemannian manifolds which is closely related to our work here. In particular, the geometric microlocal perspective, in which one compactifies complete manifolds and uses radial blowups to resolve a geometrically natural Lie algebra a vector fields and define a calculus of pseudodifferential operators, has led to index theorems on complete Riemannian manifolds with structured ends [51]. In particular there is a wealth of work (though not many index theorems) on (complete) edge differential operators (3.4), see [45]. There is, moreover, work on differential operators on singular spaces and their Fredholm and index theory from the perspective of groupoids in particular for edge differential operators [5, 30, 56]. See also [55]. The first author proved a renormalized index formula for 'Dirac-type' operators, specifically operators arising as $\operatorname{Tr} c \circ \nabla$ for $\nabla$ a Clifford-connection on a Hermitian Clifford bundle, on complete edge manifolds. The complete setting involves the subtlety that such operators, though essentially self-adjoint, are not guaranteed to be Fredholm on their natural domains. As far as the authors are aware, ours is the first index formula for the classical Dirac operator in either the incomplete edge or (complete) edge context.

\section{Connection and Dirac operator}

Let $(M, g)$ be an incomplete edge space which is spin, $\mathcal{S} \longrightarrow M$ the spinor bundle for a fixed spin structure with connection $\nabla$, and let $\partial$ be the corresponding Dirac operator. Given an orthonormal frame $e_{i}$ of the tangent bundle of $M$, the Dirac operator satisfies

$$
ð=\sum_{i} c\left(e_{i}\right) \nabla_{e_{i}}
$$

where $c(v)$ denotes Clifford multiplication by the vector $v$. See $[41,58]$ for background on spinor bundles and Dirac operators. The main goal of this section is to prove Lemma 2.2 below, where we produce a tractable form of the Dirac operator on a collar neighborhood of the boundary $\partial M$, or equivalently of the singular stratum $Y \subset X$.

\subsection{Incomplete edge metrics and their connections}

Let $M$ be the interior of a compact manifold with boundary. Assume that $\partial M=N$ participates in a fiber bundle

$$
Z \hookrightarrow N \stackrel{\phi}{\longrightarrow} Y .
$$


Let $X$ be the singular space obtained from $M$ by collapsing the fibers of the fibration $\phi$. If we want to understand the differential forms on $X$ while working on $M$, it is natural to restrict our attention to

$$
\left\{\omega \in \mathcal{C}^{\infty}\left(M ; T^{*} M\right): i_{N}^{*} \omega \in \phi^{*} \mathcal{C}^{\infty}\left(Y ; T^{*} Y\right)\right\} .
$$

Following Melrose's approach to analysis on singular spaces [49] let

$$
T_{\mathrm{ie}}^{*} M \longrightarrow M
$$

be the vector bundle whose space of sections is (2.1). We call $T_{\mathrm{ie}}^{*} M$ the 'incomplete edge cotangent bundle', and its dual bundle $T_{\mathrm{ie}} M$, the 'incomplete edge tangent bundle'. (Note that $T_{\mathrm{ie}} M$ is simply a rescaled bundle of the (complete) 'edge tangent bundle' of Mazzeo [45].) The incomplete edge tangent bundle $T_{\mathrm{ie}} M$ is canonically isomorphic to $T M$ over the interior $M^{\circ}$, but its extension to $M$ is not canonically isomorphic to $T M$ (though they are of course isomorphic bundles) as we discuss below.

Remark 2.1. The bundle $T_{\mathrm{ie}}^{*} M$ is the natural space for defining uniform ellipticity of the natural operators, such as the Hodge-Laplacian or the Dirac operator, on incomplete edge spaces. Indeed, for example, the Laplacian on functions $\Delta_{g}$ for an incomplete edge metric $g$, has principal symbol $\sigma\left(\Delta_{g}\right)$ which acts on covectors $\xi \in T^{*} M$ by $\sigma\left(\Delta_{g}\right)(\xi)=\|\xi\|_{g}^{2}$. This extends up to $\partial M$ as a function $\sigma\left(\Delta_{g}\right): T_{\mathrm{ie}}^{*} M \longrightarrow \mathbb{R}$ and is non-vanishing away from the zero section (hence uniformly elliptic). It does not extend smoothly as a function on $T^{*} M$.

Let $x$ be a boundary defining function (bdf) on $M$, meaning a smooth non-negative function $x \in \mathcal{C}^{\infty}(M ;[0, \infty))$ such that $\{x=0\}=N$ and $|d x|$ has no zeroes on $N$. Near a point $p \in \partial M$, we will typically work in local coordinates on $M$ written

$$
x, \quad y, \quad z,
$$

where $x$ is the bdf above, $y$ are coordinates along $Y$ on a neighborhood of $\phi(p)$ and $z$ are coordinates along $Z$. In particular, we choose a local trivialization of the fibration $\partial M$ on an open set $V \ni \phi(p)$, giving a local diffeomorphism $\phi^{-1}(V) \simeq V \times Z$. The $y$ and $z$ are local coordinates on the left and right factors, respectively. In local coordinates the sections of $T_{\mathrm{ie}}^{*} M$ are spanned by

$$
d x, \quad x d z, \quad d y .
$$

The crucial fact is that $x d z$ vanishes at $N$ as a section of $T^{*} M$, but it does not vanish at $N$ as a section of $T_{\mathrm{ie}}^{*} M$ because the ' $x$ ' is here part of the basis element and not a coefficient. There is an obvious map from $T_{\mathrm{ie}}^{*} M$ to $T^{*} M$ which takes a differential form expressed as a linear combination of the above basis forms to the exact same form in $T^{*} M$; this is an isomorphism on the interior $M^{\circ}$ but takes forms $x d z$ to zero over the boundary. Similarly, in local coordinates the sections of $T_{\mathrm{ie}} M$ are spanned by

$$
\partial_{x}, \quad \frac{1}{x} \partial_{z}, \quad \partial_{y},
$$

and, in contrast to $T M$, the vector field $\frac{1}{x} \partial_{z}$ is defined at $N$ as a section of $T_{\mathrm{ie}} M$.

Next consider a metric on $M$ that reflects the collapse of the fibers of $\phi$. Let $\mathscr{C}$ be a collar neighborhood of $N$ in $M$ compatible with $x, \mathscr{C} \cong[0,1]_{x} \times N$.

A product-type incomplete edge metricis a Riemannian metric on $M$ that on $\mathscr{C}$ (i.e., for some boundary defining function $x$ ) has the form

$$
g_{\mathrm{ie}}=d x^{2}+x^{2} g_{Z}+\phi^{*} g_{Y},
$$


where $g_{Z}+\phi^{*} g_{Y}$ is a submersion metric for $\phi$ independent of $x$. Note that this metric naturally induces a bundle metric on $T_{\mathrm{ie}} M$ with the advantage that it extends non-degenerately up to $\partial M$. We will consider this as a metric on $T_{\mathrm{ie}} M$ from now on. (A general incomplete edge metric is simply a bundle metric on $T_{\mathrm{ie}} M \longrightarrow M$.)

An exact ie-metric induces an orthogonal splitting

$$
T \mathscr{C}=\left\langle\partial_{x}\right\rangle \oplus T N / Y \oplus \phi^{*} T Y
$$

defined by noting that $\left\langle\partial_{x}\right\rangle \oplus T N / Y$ is the kernel of the $\phi_{*}: T \mathscr{C} \longrightarrow T Y$ and thus the perpendicular space is isomorphic to $\phi^{*} T Y$

To describe the asymptotics of the Levi-Civita connection of $g_{\text {ie }}$, let us start by recalling the behavior of the Levi-Civita connection of a submersion metric. Endow $N=\partial M$ with a submersion metric of the form $g_{N}=\phi^{*} g_{Y}+g_{Z}$. Given a vector field $U$ on $Y$, let us denote its horizontal lift to $N$ by $\widetilde{U}$. Also let us denote the projections onto each summand by

$$
\mathbf{h}: T N \longrightarrow \phi^{*} T Y, \quad \mathbf{v}: T N \longrightarrow T N / Y \text {. }
$$

The connection $\nabla^{N}$ differs from the connections $\nabla^{Y}$ on the base and the connections $\nabla^{N / Y}$ on the fibers through two tensors. The second fundamental form of the fibers is defined by

$$
\mathcal{S}^{\phi}: T N / Y \times T N / Y \longrightarrow \phi^{*} T Y, \quad \mathcal{S}^{\phi}\left(V_{1}, V_{2}\right)=\mathbf{h}\left(\nabla_{V_{1}}^{N / Y} V_{2}\right)
$$

and the curvature of the fibration is defined by

$$
\mathcal{R}^{\phi}: \phi^{*} T Y \times \phi^{*} T Y \longrightarrow T N / Y, \quad \mathcal{R}^{\phi}\left(\widetilde{U}_{1}, \widetilde{U}_{2}\right)=\mathbf{v}\left(\left[\widetilde{U}_{1}, \widetilde{U}_{2}\right]\right) .
$$

The behavior of the Levi-Civita connection (cf. [36, Proposition 13]) is then summed up in the table:

\begin{tabular}{|c||c|c|}
\hline$g_{N}\left(\nabla_{W_{1}}^{N} W_{2}, W_{3}\right)$ & $V_{0}$ & $\widetilde{U}_{0}$ \\
\hline \hline$\nabla_{V_{1}}^{N} V_{2}$ & $g_{N / Y}\left(\nabla_{V_{1}}^{N / Y} V_{2}, V_{0}\right)$ & $\phi^{*} g_{Y}\left(\mathcal{S}^{\phi}\left(V_{1}, V_{2}\right), \widetilde{U}_{0}\right)$ \\
\hline$\nabla_{\widetilde{U}}^{N} V$ & $g_{N / Y}\left([\widetilde{U}, V], V_{0}\right)-\phi^{*} g_{Y}\left(\mathcal{S}^{\phi}\left(V, V_{0}\right), \widetilde{U}\right)$ & $-\frac{1}{2} g_{N / Y}\left(\mathcal{R}^{\phi}\left(\widetilde{U}, \widetilde{U}_{0}\right), V\right)$ \\
\hline$\nabla_{V}^{N} \widetilde{U}$ & $-\phi^{*} g_{Y}\left(\mathcal{S}^{\phi}\left(V, V_{0}\right), \widetilde{U}\right)$ & $\frac{1}{2} g_{N / Y}\left(\mathcal{R}^{\phi}\left(\widetilde{U}, \widetilde{U}_{0}\right), V\right)$ \\
\hline$\nabla_{\widetilde{U}_{1}}^{N} \widetilde{U}_{2}$ & $\frac{1}{2} g_{N / Y}\left(\mathcal{R}^{\phi}\left(\widetilde{U}_{1}, \widetilde{U}_{2}\right), V_{0}\right)$ & $g_{Y}\left(\nabla_{U_{1}}^{Y} U_{2}, U_{0}\right)$ \\
\hline
\end{tabular}

We want a similar description of the Levi-Civita connection of an incomplete edge metric. The splitting of the tangent bundle of $\mathscr{C}$ induces a splitting

$$
T_{\mathrm{ie}} \mathscr{C}=\left\langle\partial_{x}\right\rangle \oplus \frac{1}{x} T N / Y \oplus \phi^{*} T Y,
$$

in terms of which a convenient choice of vector fields is

$$
\partial_{x}, \quad \frac{1}{x} V, \quad \widetilde{U},
$$

where $V$ denotes a vertical vector field at $\{x=0\}$ extended trivially to $\mathscr{C}$ and $\widetilde{U}$ denotes a vector field on $Y$, lifted to $\partial M$ and then extended trivially to $\mathscr{C}$. Note that, with respect to $g_{\text {ie }}$, these three types of vector fields are orthogonal, and that their commutators satisfy

$$
\begin{aligned}
& {\left[\partial_{x}, \frac{1}{x} V\right]=-\frac{1}{x^{2}} V \in x^{-1} \mathcal{C}^{\infty}\left(\mathscr{C}, \frac{1}{x} T \partial M / Y\right), \quad\left[\partial_{x}, \widetilde{U}\right]=0,} \\
& {\left[\frac{1}{x} V_{1}, \frac{1}{x} V_{2}\right]=\frac{1}{x^{2}}\left[V_{1}, V_{2}\right] \in x^{-1} \mathcal{C}^{\infty}\left(\mathscr{C}, \frac{1}{x} T \partial M / Y\right),} \\
& {\left[\frac{1}{x} V, \widetilde{U}\right]=\frac{1}{x}[V, \widetilde{U}] \in \mathcal{C}^{\infty}\left(\mathscr{C}, \frac{1}{x} T \partial M / Y\right),} \\
& {\left[\widetilde{U}_{1}, \widetilde{U}_{2}\right] \in x \mathcal{C}^{\infty}\left(\mathscr{C}, \frac{1}{x} T \partial M / Y\right)+\mathcal{C}^{\infty}\left(\mathscr{C}, \phi^{*} T Y\right) .}
\end{aligned}
$$


The Levi-Civita connection $\nabla$ for $g_{\text {ie }}$ satisfies the Koszul formula

$$
\begin{aligned}
2 g_{\mathrm{ie}}\left(\nabla_{W_{0}} W_{1}, W_{2}\right)= & W_{0} g_{\mathrm{ie}}\left(W_{1}, W_{2}\right)+W_{1} g_{\mathrm{ie}}\left(W_{0}, W_{2}\right)-W_{2} g_{\mathrm{ie}}\left(W_{0}, W_{1}\right) \\
& +g_{\mathrm{ie}}\left(\left[W_{0}, W_{1}\right], W_{2}\right)-g_{\mathrm{ie}}\left(\left[W_{0}, W_{2}\right], W_{1}\right)-g_{\mathrm{ie}}\left(\left[W_{1}, W_{2}\right], W_{0}\right),
\end{aligned}
$$

and it is easy to see that the expression on the right hand side is smooth on all of $M$ for any any smooth vector field $W_{0} \in C^{\infty}(M ; T M)$ and $W_{1}, W_{2} \in C^{\infty}\left(M ; T_{\mathrm{ie}} M\right)$. In fact we will now describe the action of $\nabla$ in relation to the splitting of $T \mathscr{C}$ above.

If $W_{0} \in\left\{\partial_{x}, V, \widetilde{U}\right\}$ and $W_{1}, W_{2} \in\left\{\partial_{x}, \frac{1}{x} V, \widetilde{U}\right\}$ then we find

$$
\begin{aligned}
& g_{\text {ie }}\left(\nabla_{W_{0}} W_{1}, W_{2}\right)=0 \quad \text { if } \quad \partial_{x} \in\left\{W_{0}, W_{1}, W_{2}\right\} \\
& \text { except for } \quad g_{\text {ie }}\left(\nabla_{V_{1}} \partial_{x}, \frac{1}{x} V_{2}\right)=-g_{\text {ie }}\left(\nabla_{V_{1}} \frac{1}{x} V_{2}, \partial_{x}\right)=g_{Z}\left(V_{1}, V_{2}\right),
\end{aligned}
$$

and otherwise

\begin{tabular}{|c||c|c|}
\hline$g_{\mathrm{ie}}\left(\nabla_{W_{1}} W_{2}, W_{3}\right)$ & $\frac{1}{x} V_{0}$ & $\widetilde{U}_{0}$ \\
\hline \hline$\nabla_{V_{1}} \frac{1}{x} V_{2}$ & $g_{N / Y}\left(\nabla_{V_{1}}^{N / Y} V_{2}, V_{0}\right)$ & $x \phi^{*} g_{Y}\left(\mathcal{S}^{\phi}\left(V_{1}, V_{2}\right), \widetilde{U}_{0}\right)$ \\
\hline$\nabla_{\widetilde{U}} \frac{1}{x} V$ & $g_{N / Y}\left([\widetilde{U}, V], V_{0}\right)-\phi^{*} g_{Y}\left(\mathcal{S}^{\phi}\left(V, V_{0}\right), \widetilde{U}\right)$ & $-\frac{x}{2} g_{N / Y}\left(\mathcal{R}^{\phi}\left(\widetilde{U}, \widetilde{U}_{0}\right), V\right)$ \\
\hline$\nabla_{V} \widetilde{U}$ & $-x \phi^{*} g_{Y}\left(\mathcal{S}^{\phi}\left(V, V_{0}\right), \widetilde{U}\right)$ & $\frac{x^{2}}{2} g_{N / Y}\left(\mathcal{R}^{\phi}\left(\widetilde{U}, \widetilde{U}_{0}\right), V\right)$ \\
\hline$\nabla_{\widetilde{U}_{1}} \widetilde{U}_{2}$ & $\frac{x}{2} g_{N / Y}\left(\mathcal{R}^{\phi}\left(\widetilde{U}_{1}, \widetilde{U}_{2}\right), V_{0}\right)$ & $g_{Y}\left(\nabla_{U_{1}}^{Y} U_{2}, U_{0}\right)$ \\
\hline
\end{tabular}

We point out a few consequences of these computations. First note that

$$
\nabla: \mathcal{C}^{\infty}\left(M ; T_{\mathrm{ie}} M\right) \longrightarrow \mathcal{C}^{\infty}\left(M ; T^{*} M \otimes T_{\mathrm{ie}} M\right)
$$

defines a connection on the incomplete edge tangent bundle. Also note that this connection asymptotically preserves the splitting of $T_{\mathrm{ie}} \mathscr{C}$ into two bundles

$$
T_{\mathrm{ie}} \mathscr{C}=\left[\left\langle\partial_{x}\right\rangle \oplus \frac{1}{x} T N / Y\right] \oplus \phi^{*} T Y
$$

in that if $W_{1}, W_{2} \in \mathcal{V}_{\text {ie }}$ are sections of the two different summands then

$$
g_{\mathrm{ie}}\left(\nabla_{W_{0}} W_{1}, W_{2}\right)=\mathcal{O}(x) \quad \text { for all } \quad W_{0} \in \mathcal{C}^{\infty}(M ; T M) .
$$

In fact, let us denote the projections onto each summand of (2.6) by

$$
\mathbf{v}_{+}: T_{\mathrm{ie}} \mathscr{C} \longrightarrow\left\langle\partial_{x}\right\rangle \oplus \frac{1}{x} T N / Y, \quad \mathbf{h}: T_{\mathrm{ie}} \mathscr{C} \longrightarrow \phi^{*} T Y,
$$

and define connections

$$
\begin{aligned}
& \nabla^{v_{+}}=\mathbf{v}_{+} \circ \nabla \circ \mathbf{v}_{+}: \mathcal{C}^{\infty}\left(\mathscr{C} ;\left\langle\partial_{x}\right\rangle \oplus \frac{1}{x} T N / Y\right) \longrightarrow \mathcal{C}^{\infty}\left(\mathscr{C} ; T^{*} \mathscr{C} \otimes\left(\left\langle\partial_{x}\right\rangle \oplus \frac{1}{x} T N / Y\right)\right), \\
& \nabla^{h}=\phi^{*} \nabla^{Y}: \mathcal{C}^{\infty}\left(\mathscr{C} ; \phi^{*} T Y\right) \longrightarrow \mathcal{C}^{\infty}\left(\mathscr{C} ; T^{*} \mathscr{C} \otimes \phi^{*} T Y\right) .
\end{aligned}
$$

Denote by

$$
j_{\varepsilon}:\{x=\varepsilon\} \hookrightarrow \mathscr{C}
$$

the inclusion, and identify $\{x=\varepsilon\}$ with $N=\{x=0\}$, note that the pull-back connections $j_{\varepsilon}^{*} \nabla^{v_{+}}$ and $j_{\varepsilon}^{*} \nabla^{h}$ are independent of $\varepsilon$ and

$$
j_{0}^{*} \nabla=j_{0}^{*} \nabla^{v_{+}} \oplus j_{0}^{*} \nabla^{h} .
$$


In terms of the local connection one-form $\omega$ and the splitting (2.6), we have

$$
\begin{aligned}
& P^{V^{+}} \omega=\left(\begin{array}{cc}
\omega_{N / Y} & \mathcal{O}(x) \\
\mathcal{O}(x) & \mathcal{O}\left(x^{2}\right)
\end{array}\right), \quad P^{H} \omega=\left(\begin{array}{cc}
\omega_{\mathcal{S}} & \mathcal{O}(x) \\
\mathcal{O}(x) & \phi^{*} \omega_{Y}
\end{array}\right), \\
& \omega=\left(\begin{array}{cc}
\omega_{v_{+}} & \mathcal{O}(x) \\
\mathcal{O}(x) & \phi^{*} \omega_{Y}+\mathcal{O}\left(x^{2}\right)
\end{array}\right),
\end{aligned}
$$

where $P^{V^{+}} \omega$ is the projection onto the dual bundle of $\left[\left\langle\partial_{x}\right\rangle \oplus \frac{1}{x} T N / Y\right], P^{H} \omega$ is the projection onto the dual bundle of $\phi^{*} T Y$, and the forms $\omega_{N / Y}, \omega_{\mathcal{S}}, \omega_{Y}, \omega_{v+}$ are defined by these equations. Finally, consider the curvature $R_{\text {ie }}$ of $\nabla$. If $W_{1}, W_{2} \in \mathcal{V}_{\text {ie }}$ are sections of two different summands of $(2.6)$ and $W_{3}, W_{4} \in \mathcal{C}^{\infty}\left(\mathscr{C}, T N / Y \oplus \phi^{*} T Y\right)$ then

$$
g_{\text {ie }}\left(R_{\text {ie }}\left(W_{3}, W_{4}\right) W_{1}, W_{2}\right)=\mathcal{O}(x),
$$

but

$$
g_{\mathrm{ie}}\left(R_{\mathrm{ie}}\left(\partial_{x}, W_{4}\right) W_{1}, W_{2}\right)=\frac{1}{x} g_{\mathrm{ie}}\left(\nabla_{W_{4}} W_{1}, W_{2}\right)=\mathcal{O}(1)
$$

We will be interested in the curvature along the level sets of $x$. Schematically, if $\Omega$ denotes the $\operatorname{End}\left(T_{\mathrm{ie}} M\right)$-valued two-form corresponding to the curvature of $\nabla$, then with respect to the splitting (2.6) we have

$$
\left.\Omega\right|_{x=\varepsilon}=\left(\begin{array}{cc}
\Omega_{v_{+}} & \mathcal{O}(\varepsilon) \\
\mathcal{O}(\varepsilon) & \phi^{*} \Omega_{Y}
\end{array}\right)
$$

where $\Omega_{v_{+}}$is the tangential curvature associated to $\omega_{N / Y}+\omega_{\mathcal{S}}$ and $\Omega_{Y}$ is the curvature associated to $\omega_{Y}$, and analogously to $(2.7)$,

$$
j_{0}^{*} \Omega=j_{0}^{*} \Omega_{v_{+}}+\phi^{*} \Omega_{Y} .
$$

Following [13] and [36], it will be convenient to use the block-diagonal connection $\widetilde{\nabla}$ on $T_{\mathrm{ie}} M$ from the splitting (2.5). Thus

$$
\widetilde{\nabla}: \mathcal{C}^{\infty}\left(\mathscr{C}, T_{\mathrm{ie}} \mathscr{C}\right) \longrightarrow \mathcal{C}^{\infty}\left(\mathscr{C} ; T^{*} \mathscr{C} \otimes T_{\mathrm{ie}} \mathscr{C}\right)
$$

satisfies

$$
\widetilde{\nabla} \partial_{x}=0, \quad \widetilde{\nabla}_{\partial_{x}}=0, \quad \text { and }
$$

\begin{tabular}{|c||c|c|}
\hline$g_{\mathrm{ie}}\left(\widetilde{\nabla}_{W_{1}} W_{2}, W_{3}\right)$ & $\frac{1}{x} V_{0}$ & $\widetilde{U}_{0}$ \\
\hline \hline$\widetilde{\nabla}_{V_{1}} \frac{1}{x} V_{2}$ & $g_{N / Y}\left(\nabla_{V_{1}}^{N / Y} V_{2}, V_{0}\right)$ & 0 \\
\hline$\widetilde{\nabla}_{\widetilde{U}} \frac{1}{x} V$ & $g_{N / Y}\left([\widetilde{U}, V], V_{0}\right)-\phi^{*} g_{Y}\left(\mathcal{S}^{\phi}\left(V, V_{0}\right), \widetilde{U}\right)$ & 0 \\
\hline$\widetilde{\nabla}_{V} \widetilde{U}$ & 0 & 0 \\
\hline$\widetilde{\nabla}_{\widetilde{U}_{1}} \widetilde{U}_{2}$ & 0 & $g_{Y}\left(\nabla_{U_{1}}^{Y} U_{2}, U_{0}\right)$ \\
\hline
\end{tabular}

The connection $\widetilde{\nabla}$ is a metric connection and preserves the splitting (2.6). 


\subsection{Clifford bundles and Clifford actions}

The incomplete edge Clifford bundle, denoted $\mathrm{Cl}_{\mathrm{ie}}(M, g)$, is the bundle obtained by taking the Clifford algebra of each fiber of $T_{\mathrm{ie}} M$. Concretely,

$$
\mathrm{Cl}_{\mathrm{ie}}(M, g)=\sum_{k=0}^{\infty} T_{\mathrm{ie}} M^{\otimes k} /\left(x \otimes y+y \otimes x=-2\langle x, y\rangle_{g}\right) .
$$

This is a smooth vector bundle on all of $M$.

We assume that $M$ is spin and fix a spin bundle $\mathcal{S} \longrightarrow M$. Note that $\mathcal{S}$ is indeed a smooth vector bundle on all of $M$ (including the boundary) as the orthonormal frame bundle $\mathcal{O} \longrightarrow M^{\circ}$ extends smoothly up to the boundary (indeed, consider the local orthonormal frames from the previous section). In fact, the orhonormal frame bundle of $T_{\mathrm{ie}} M$ gives the extension of $\mathcal{O}$ to $M$. Denote Clifford multiplication, which also extends smoothly to all incomplete edge vector fields, by

$$
c: \mathcal{C}^{\infty}\left(M, T_{\mathrm{ie}} M\right) \longrightarrow \mathcal{C}^{\infty}(M ; \operatorname{End}(\mathcal{S})) .
$$

We denote the connection induced on $\mathcal{S}$ by the Levi-Civita connection $\nabla$ by the same symbol. Let $\partial$ denote the corresponding Dirac operator.

Lemma 2.2. Let $\mathscr{C} \cong[0,1)_{x} \times N$ be a collar neighborhood of the boundary. Let

$$
\partial_{x}, \quad \frac{1}{x} V_{\alpha}, \quad \widetilde{U}_{i}
$$

denote a local orthonormal frame consistent with the splitting (2.5). In terms of this frame and the connection $\widetilde{\nabla}$ from (2.10), the Dirac operator $\partial$ decomposes as

$$
\partial=c\left(\partial_{x}\right) \partial_{x}+\frac{f}{2 x} c\left(\partial_{x}\right)+\frac{1}{x} \sum_{\alpha=1}^{f} c\left(\frac{1}{x} V_{\alpha}\right) \widetilde{\nabla}_{V_{\alpha}}+\sum_{i=1}^{b} c\left(\widetilde{U}_{i}\right) \widetilde{\nabla}_{\widetilde{U}_{i}}+B,
$$

where $f=\operatorname{dim} Z, b=\operatorname{dim} Y$, and $B \in \mathcal{C}^{\infty}(M, \operatorname{End}(\mathcal{S}))$.

Proof. Consider the difference of connections (on the tangent bundle)

$$
A=\nabla-\widetilde{\nabla} \in \mathcal{C}^{\infty}\left(\mathscr{C} ; T^{*} M \otimes \operatorname{End}\left({ }^{\mathrm{ie}} T \mathscr{C}\right)\right) .
$$

From [16] we have

$$
\text { ə }=\sum_{i} c\left(e_{i}\right)\left(\widetilde{\nabla}_{e_{i}}+\widetilde{A}\left(e_{i}\right)\right)
$$

where $\widetilde{A}(W):=\frac{1}{4} \sum_{j k} g_{\mathrm{ie}}\left(A(W) e_{j}, e_{k}\right) c\left(e_{j}\right) c\left(e_{k}\right)$. From Section 2.1 we have

$$
\begin{aligned}
& g_{\text {ie }}\left(A\left(W_{0}\right) W_{1}, W_{2}\right)=0 \quad \text { if } \quad \partial_{x} \in\left\{W_{0}, W_{1}, W_{2}\right\} \\
& \text { except for } \quad g_{\text {ie }}\left(A\left(\frac{1}{x} V_{\alpha}\right) \partial_{x}, \frac{1}{x} V_{\beta}\right)=-g_{\text {ie }}\left(\frac{1}{x} A\left(V_{\alpha}\right) \frac{1}{x} V_{\beta}, \partial_{x}\right)=\frac{1}{x} g_{Z}\left(V_{\alpha}, V_{\beta}\right),
\end{aligned}
$$

and otherwise

\begin{tabular}{|c||c|c|}
\hline$g_{\mathrm{ie}}\left(A\left(W_{1}\right) W_{2}, W_{3}\right)$ & $\frac{1}{x} V_{0}$ & $\widetilde{U}_{0}$ \\
\hline \hline$A\left(\frac{1}{x} V_{1}\right) \frac{1}{x} V_{2}$ & 0 & $\phi^{*} g_{Y}\left(\mathcal{S}^{\phi}\left(V_{1}, V_{2}\right), \widetilde{U}_{0}\right)$ \\
\hline$A(\widetilde{U}) \frac{1}{x} V$ & 0 & $-\frac{x}{2} g_{N / Y}\left(\mathcal{R}^{\phi}\left(\widetilde{U}, \widetilde{U}_{0}\right), V\right)$ \\
\hline$A\left(\frac{1}{x} V\right) \widetilde{U}$ & $-\phi^{*} g_{Y}\left(\mathcal{S}^{\phi}\left(V, V_{0}\right), \widetilde{U}\right)$ & $\frac{x}{2} g_{N / Y}\left(\mathcal{R}^{\phi}\left(\widetilde{U}, \widetilde{U}_{0}\right), V\right)$ \\
\hline$A\left(\widetilde{U}_{1}\right) \widetilde{U}_{2}$ & $\frac{x}{2} g_{N / Y}\left(\mathcal{R}^{\phi}\left(\widetilde{U}_{1}, \widetilde{U}_{2}\right), V_{0}\right)$ & 0 \\
\hline
\end{tabular}


Hence $\frac{1}{4} \sum_{s, t, u} g_{\mathrm{ie}}\left(A\left(e_{s}\right) e_{t}, e_{u}\right) c\left(e_{s}\right) c\left(e_{t}\right) c\left(e_{u}\right)$ has terms of order $\mathcal{O}\left(\frac{1}{x}\right), \mathcal{O}(1)$, and $\mathcal{O}(x)$. The terms of order $\frac{1}{x}$ are

$$
\begin{aligned}
\frac{1}{4} \sum_{\alpha} & \left(g_{\mathrm{ie}}\left(A\left(\frac{1}{x} V_{\alpha}\right) \partial_{x}, \frac{1}{x} V_{\alpha}\right) c\left(\partial_{x}\right)+g_{\mathrm{ie}}\left(A\left(\frac{1}{x} V_{\alpha}\right) \frac{1}{x} V_{\alpha}, \partial_{x}\right)\left(-c\left(\partial_{x}\right)\right)\right) \\
= & \frac{1}{4} \sum_{\alpha} 2 \frac{c\left(\partial_{x}\right)}{x}=\frac{f}{2 x} c\left(\partial_{x}\right),
\end{aligned}
$$

which establishes (2.12).

\subsection{The APS boundary projection}

We now define the APS boundary condition discussed in the introduction. We will make use of a simplified coordinate system near the boundary of $M$, namely, let $\left(x, x^{\prime}\right)$ be coordinates near a point on $\partial M$ for which $x^{\prime} \in \mathbb{R}^{n-1}$ are coordinates on $\partial M$ and $x$ is the same fixed boundary defining function used in (2.3). For the cutoff manifold $M_{\varepsilon}=\{x \geq \varepsilon\}$, consider the differential operator on sections of $\mathcal{S}$ over $\partial M_{\varepsilon}$ defined by choosing any orthonormal frame $e_{p}$, $p=1, \ldots, n-1$ of the distribution of the tangent bundle orthogonal to $\partial_{x}$ and setting

$$
\frac{1}{\varepsilon} \widetilde{\widetilde{\partial}}_{\varepsilon}:=-\left.c\left(\partial_{x}\right)\left(\sum_{p=1}^{n-1} c\left(e_{p}\right) \widetilde{\nabla}_{e_{p}}\right)\right|_{x=\varepsilon},
$$

where $\widetilde{\nabla}$ is the connection from $(2.10)$. The operator $\widetilde{\partial}_{\varepsilon}$ is defined independently of the choice of frame, so we may take frames as in (2.11) to obtain

$$
\widetilde{\widetilde{\partial}}_{\varepsilon}=-\left.x c\left(\partial_{x}\right)\left(\sum_{\alpha=1}^{f} c\left(\frac{1}{x} V_{\alpha}\right) \widetilde{\nabla}_{\frac{1}{x} V_{\alpha}}+\sum_{j=1}^{b} c\left(\widetilde{U}_{j}\right) \widetilde{\nabla}_{\widetilde{U}_{j}}\right)\right|_{x=\varepsilon} .
$$

We refer to $\widetilde{\partial}_{\varepsilon}$ below as the tangential operator, since for every $\varepsilon$ it acts tangentially along the boundary $\partial M_{\varepsilon}$. The operator $\widetilde{\widetilde{\partial}}_{\varepsilon}$ is self-adjoint on $L^{2}\left(\partial M_{\varepsilon}, \mathcal{S}\right)$.

We denote the dual coordinates on $T_{x, x^{\prime}}^{*} M$ by $\left(\xi, \xi^{\prime}\right)$. Using the identification of $T^{*} M$ with $T M$ induced by the metric $g$, the principal symbol of $\partial$ is given by

$$
\sigma(ð)\left(x, x^{\prime}\right)=i \xi c\left(\partial_{x}\right)+i c\left(\xi^{\prime} \cdot \partial_{x^{\prime}}\right),
$$

where $\xi^{\prime} \cdot \partial_{x^{\prime}}=\sum_{i=1}^{n-1} \xi_{j}^{\prime} \partial_{x_{j}^{\prime}}$. Note that using coordinates as in $(2.14)$, for $x=\varepsilon$, $\widetilde{\widetilde{d}}_{\varepsilon}$ has principal symbol

$$
\sigma\left(\widetilde{\widetilde{\partial}}_{\varepsilon}\right)\left(x^{\prime}, \xi^{\prime}\right)=-i \varepsilon c\left(\partial_{x}\right) c\left(\xi^{\prime} \cdot \partial_{x^{\prime}}\right) .
$$

Since $\sigma\left(\widetilde{\widetilde{\partial}}_{\varepsilon}\right)\left(x^{\prime}, \xi^{\prime}\right)^{2}=\varepsilon^{2}\left|\left(0, \xi^{\prime}\right)\right|_{g}^{2}$, if we define $\widehat{\xi}^{\prime}=\xi^{\prime} /\left|\left(0, \xi^{\prime}\right)\right|_{g}$, then

$$
\sigma\left(\widetilde{\partial}_{\varepsilon}\right)\left(x^{\prime}, \xi^{\prime}\right)=\left|\left(0, \xi^{\prime}\right)\right|_{g} \sigma\left(\widetilde{\partial}_{\varepsilon}\right)\left(x^{\prime}, \widehat{\xi}^{\prime}\right)=\varepsilon\left|\left(0, \xi^{\prime}\right)\right|_{g}\left(\pi_{\varepsilon,+, \widehat{\xi}^{\prime}}\left(x^{\prime}\right)-\pi_{\varepsilon,-, \widehat{\xi}^{\prime}}\left(x^{\prime}\right)\right),
$$

where $\pi_{\varepsilon, \pm, \widehat{\xi}^{\prime}}\left(x^{\prime}\right)$ are orthogonal projections onto \pm eigenspaces of $\sigma\left(\widetilde{\widetilde{d}}_{\varepsilon}\right)\left(x^{\prime}, \widehat{\xi}^{\prime}\right)$. We will define a boundary condition for $\partial$ on the cutoff manifolds $M_{\varepsilon}$,

$$
\pi_{\mathrm{APS}, \varepsilon}:=L^{2} \quad \text { orthogonal projection onto } V_{-, \varepsilon},
$$

where $V_{-, \varepsilon}$ is the direct sum of eigenspaces of $\widetilde{\widetilde{d}}_{\varepsilon}$ with negative eigenvalues. We recall basic facts about $\pi_{\mathrm{APS}, \varepsilon}$. 
Theorem 2.3 ([8, 59]). For fixed $\varepsilon$, the operator $\pi_{\mathrm{APS}, \varepsilon}$ is a pseudodifferential operator of order 0 , i.e., $\pi_{\mathrm{APS}, \varepsilon} \in \Psi^{0}\left(\partial M_{\varepsilon} ; \mathcal{S}\right)$. Its principal symbol satisfies

$$
\sigma\left(\pi_{\mathrm{APS}, \varepsilon}\right)\left(x^{\prime}, \xi^{\prime}\right)=\pi_{\varepsilon,-\widehat{\xi}^{\prime}}\left(x^{\prime}\right),
$$

where $\pi_{\varepsilon,-, \widehat{\xi}^{\prime}}\left(x^{\prime}\right)$ is projection onto the negative eigenspace of $-i c\left(\partial_{x}\right) c\left(\widehat{\xi}^{\prime} \cdot \partial_{x^{\prime}}\right)$ from $(2.15)$.

Consider the domains for $ð$ on $L^{2}\left(M_{\varepsilon} ; \mathcal{S}\right)$ defined as follows

$$
\begin{aligned}
& \mathcal{D}_{\mathrm{APS}, \varepsilon}:=\left\{u \in H^{1}\left(M_{\varepsilon}, \mathcal{S}\right):\left(\mathrm{Id}-\pi_{\mathrm{APS}, \varepsilon}\right) u=0\right\}, \\
& \mathcal{D}_{\mathrm{APS}, \varepsilon}^{+}:=\left\{u \in \mathcal{D}_{\mathrm{APS}, \varepsilon}: \operatorname{image}(u) \subset \mathcal{S}^{+}\right\} .
\end{aligned}
$$

In one of the main results of this paper, we will show that $\partial$ has a unique self-adjoint extension $\mathcal{D}$, such that for $\varepsilon>0$ sufficiently small,

$$
\text { Ind }\left(\text { ð: } \mathcal{D}_{\mathrm{APS}, \varepsilon}^{+} \longrightarrow L^{2}\left(M_{\varepsilon} ; \mathcal{S}^{-}\right)\right)=\operatorname{Ind}\left(\text { ठ: } \mathcal{D}^{+} \longrightarrow L^{2}\left(M ; \mathcal{S}^{-}\right)\right) \text {, }
$$

where $\mathcal{D}^{+}$the elements of $\mathcal{D}$ valued in $\mathcal{S}^{+}$. Indeed, this will follow from Theorems 4.1 and 5.1 below.

\section{Mapping properties of $ð$}

In this section we will use the results and techniques in $[2,3]$ to prove Theorem 1.1. We proceed by constructing a parametrix for $\partial$ and analyzing the mapping properties of this parametrix.

Let $\mathcal{D}$ denote the domain of the unique self-adjoint extention of $\partial$. At the end of this section, we analyze the structure of the generalized inverse $Q$ for $\partial$, that is, the map

$$
Q: L^{2}(M ; \mathcal{S}) \longrightarrow \mathcal{D} \quad \text { satisfying } \quad \partial Q=\mathrm{Id}-\pi_{\text {ker }} \quad \text { and } \quad Q=Q^{*},
$$

where $\pi_{\text {ker }}$ is $L^{2}$-orthogonal projection onto the kernel of $\partial$. Here the adjoint $Q^{*}$ is taken with respect to the pairing defined for sections $\phi, \psi$ by

$$
\langle\phi, \psi\rangle_{L^{2}}=\int\langle\phi, \psi\rangle_{G} d \mathrm{Vol}_{g},
$$

where $G$ is the Hermitian inner product on $\mathcal{S}$.

\subsection{The "geometric Witt condition"}

The proof of Theorem 1.1 relies on an assumption on an induced family of Dirac operators on the fiber $Z$ which we describe now. By Lemma 2.2, on a collar neighborhood of the boundary,

$$
\mathcal{U} \subset M \quad \text { with } \mathcal{U} \simeq\left[0, \varepsilon_{0}\right)_{x} \times \partial M
$$

we can write

$$
\check{\partial}=c\left(\partial_{x}\right)\left(\partial_{x}+\frac{f}{2 x}+\frac{1}{x} \widetilde{\partial}_{y}^{Z}-\sum_{i=1}^{b} c\left(\partial_{x}\right) c\left(\widetilde{U}_{i}\right) \widetilde{\nabla}_{\widetilde{U}_{i}}\right)+B
$$

with $\|B\|=O(1)$ and where, for $y$ in the base $Y$

$$
\widetilde{\partial}_{y}^{Z}=-c\left(\partial_{x}\right) \cdot \sum_{\alpha=1}^{f} c\left(\frac{1}{x} V_{\alpha}\right) \cdot \widetilde{\nabla}_{V_{\alpha}}
$$

The operator $\mathrm{d}_{y}^{Z}$ defines a self-adjoint operator on the fiber over $y \in Y$ in the boundary fibration $N \stackrel{\phi}{\longrightarrow} Y$ acting on sections of the restriction of the spin bundle $\mathcal{S}_{y}$.

We will assume the following "geometric Witt condition" discussed in the introduction. 
Assumption 3.1. The fiber operator $\varlimsup_{y}^{Z}$ in (3.3) satisfies

$$
(-1 / 2,1 / 2) \cap \operatorname{spec}\left(\varlimsup_{y}^{Z}\right)=\varnothing \quad \text { for all } y \text {. }
$$

\subsection{Review of edge and incomplete edge operators}

A vector field on $M$ is an 'edge vector field' if its restriction to $N=\partial M$ is tangent to the fibers of $\phi$ [45]. A differential operator is an edge differential operator if in every coordinate chart it can be written as a polynomial in edge vector fields. Thus if $E$ and $F$ are vector bundles over $M$, we say that $P^{\prime}$ is an $m^{\text {th }}$ order edge differential operator between sections of $E$ and $F$, denoted $P^{\prime} \in \operatorname{Diff}_{\mathrm{e}}^{m}(M ; E, F)$, if in local coordinates we have

$$
P^{\prime}=\sum_{j+|\alpha|+|\gamma| \leq m} a_{j, \alpha, \gamma}(x, y, z)\left(x \partial_{x}\right)^{j}\left(x \partial_{y}\right)^{\alpha}\left(\partial_{z}\right)^{\gamma}
$$

where $\alpha$ denotes a multi-index $\left(\alpha_{1}, \ldots, \alpha_{b}\right)$ with $|\alpha|=\alpha_{1}+\cdots+\alpha_{b}$ and similarly for $\gamma=$ $\left(\gamma_{1}, \ldots, \gamma_{f}\right)$, and each $a_{j, \alpha, \gamma}(x, y, z)$ is a local section of $\operatorname{hom}(E, F)$.

A differential operator $P$ is an 'incomplete edge differential operator' of order $m$ if $P^{\prime}=x^{m} P$ is an edge differential operator of order $m$. Thus, symbolically,

$$
\operatorname{Diff}_{\mathrm{ie}}^{m}(M ; E, F)=x^{-m} \operatorname{Diff}_{\mathrm{e}}^{m}(M ; E, F),
$$

and in local coordinates

$$
P=x^{-m} \sum_{j+|\alpha|+|\gamma| \leq m} a_{j, \alpha, \gamma}(x, y, z)\left(x \partial_{x}\right)^{j}\left(x \partial_{y}\right)^{\alpha}\left(\partial_{z}\right)^{\gamma} .
$$

The (incomplete edge) principal symbol of $P$ is defined on the incomplete edge cotangent bundle,

$$
\sigma(P) \in \mathcal{C}^{\infty}\left(T_{\mathrm{ie}}^{*} M ; \pi^{*} \operatorname{hom}(E, F)\right)
$$

where $\pi: T_{\mathrm{ie}}^{*} M \longrightarrow M$ denotes the bundle projection. In local coordinates it is given by

$$
\sigma(P)(x, y, z, \xi, \eta, \zeta):=\sum_{j+|\alpha|+|\gamma|=m} a_{j, \alpha, \gamma}(x, y, z)(\xi)^{j}(\eta)^{\alpha}(\zeta)^{\gamma}
$$

We say that $P$ is elliptic if this symbol is invertible whenever $(\xi, \eta, \zeta) \neq 0$.

Lemma 3.2. The Dirac operator $\partial$ on an incomplete edge space is an elliptic incomplete edge differential operator of order 1 , i.e., is an elliptic element of $\operatorname{Diff}_{\mathrm{ie}}^{1}(M ; \mathcal{S})$. In particular, $x \precsim$ is an elliptic element of $\operatorname{Diff}_{\mathrm{e}}^{1}(M ; \mathcal{S})$.

Proof. This follows from equation (2.12) in Lemma 2.2.

\subsection{Parametrix of $x ð$ on weighted edge spaces}

Lemma 3.2 shows that $x ð$ is an elliptic edge operator. By the theory of edge operators [45], this implies that $x$ ə is a bounded operator between appropriate weighted Sobolev spaces, whose definition we now recall.

Let $\mathcal{D}^{\prime}(M ; \mathcal{S})$ denote distributional sections. Given $k \in \mathbb{N}$, let

$$
H_{\mathrm{e}}^{k}(M ; \mathcal{S}):=\left\{u \in \mathcal{D}^{\prime}(M ; \mathcal{S}): A^{1} \cdots A^{j} u \in L^{2}(M ; \mathcal{S}) \text { for } j \leq k \text { and } A^{i} \in \operatorname{Diff}_{\mathrm{e}}^{1}(M ; \mathcal{S})\right\} .
$$


In particular, $u \in H_{\mathrm{e}}^{1}(M ; \mathcal{S})$ if and only if $u \in L^{2}(M ; \mathcal{S})$ and, for any edge vector field $V \in$ $C^{\infty}\left(M ; T_{\mathrm{e}} M\right), \nabla_{V} u \in L^{2}(M ; \mathcal{S})$. The weighted edge Sobolev spaces are defined by

$$
x^{\delta} H_{\mathrm{e}}^{k}(M ; \mathcal{S}):=\left\{u: x^{-\delta} u \in H_{\mathrm{e}}^{k}(M ; \mathcal{S})\right\} .
$$

Thus, the map

$$
x ð: x^{\delta} H_{\mathrm{e}}^{k}(M ; \mathcal{S}) \longrightarrow x^{\delta} H^{k-1}(M ; \mathcal{S})
$$

is bounded for all $\delta \in \mathbb{R}, k \in \mathbb{N}$, in fact for $k \in \mathbb{R}$ by duality and interpolation. We will prove the following

Proposition 3.3. Under the Witt assumption (Assumption 3.1), the map (3.5) is Fredholm for $0<\delta<1$.

Remark 3.4. Recall that the space $L^{2}(M ; \mathcal{S})$ used to define the $x^{\delta} H^{k}(M ; \mathcal{S})$ is equipped with the inner product from the Hermitian metric on $\mathcal{S}$ and the volume form of the incomplete edge metric $g$.

Remark 3.5 (complete edge manifolds). We now give a rough sketch of how one can use the main theorem of this paper to prove an index formula for Dirac operators in the complete edge case, leaving the details to the reader. Note that for each incomplete edge metric $g$ on $M$ as above, there is an edge metric $\widetilde{g}$ defined by

$$
g=x^{-2} \widetilde{g}
$$

where $x$ remains a boundary defining function as above, and in particular is non-vanishing in the interior of $M$. Choosing a spin structure, the Dirac operator of $g$ is related to that of $\widetilde{g}$ (under a natural identification of the spin bundles) by

$$
\mathrm{\partial}_{\widetilde{g}}=x^{(n+1) / 2} \mathrm{\partial}_{\widetilde{g}} x^{-(n-1) / 2} .
$$

One can show that, under the strengthened assumption, $\mathrm{\partial}_{\tilde{g}}: H_{\mathrm{e}}^{1}(M ; \mathcal{S}) \longrightarrow L^{2}(M ; \mathcal{S})$ is Fredholm (i.e., one can take the weight $\delta=0$ ) and that the index of this map is equal to that of $\partial_{g}$. The main theorem then gives an index formula for $\partial_{\widetilde{g}}$ in this case.

In fact we will need more than Proposition 3.3; the proof of the Main Theorem requires a detailed understanding of the structure of parametrices for $x$ ठ. To understand these, we must recall of edge double space $M_{\mathrm{e}}^{2}$, depicted heuristically in Fig. 3 below, and edge pseudodifferential operators, defined in [45] with background material in [53]. The edge double space $M_{\mathrm{e}}^{2}$ is a manifold with corners, obtained by radial blowup of $M \times M$, namely $M_{\mathrm{e}}^{2}:=\left[M \times M ; \operatorname{diag}_{\mathrm{fib}}(\partial M \times\right.$ $\partial M)$ ], where the notation is that in [53]. Here $\operatorname{diag}_{\mathrm{fib}}(\partial M \times \partial M)$ denotes the fiber diagonal

$$
\operatorname{diag}_{\text {fib }}(N \times N)=\{(p, q) \in N \times N: \phi(p)=\phi(q)\},
$$

where $\phi: N \longrightarrow Y$ is fiber bundle projection onto $Y$. Whereas $M \times M$ is a manifold with corners with two boundary hypersurfaces, $M_{\mathrm{e}}^{2}$ has a third boundary hypersurface introduced by the blowup.

Let ff be the boundary hypersurface of $M_{\mathrm{e}}^{2}$ introducedby the blowup.

Furthermore we have a blowdown map.

$$
\beta: M_{\mathrm{e}}^{2} \longrightarrow M \times M,
$$




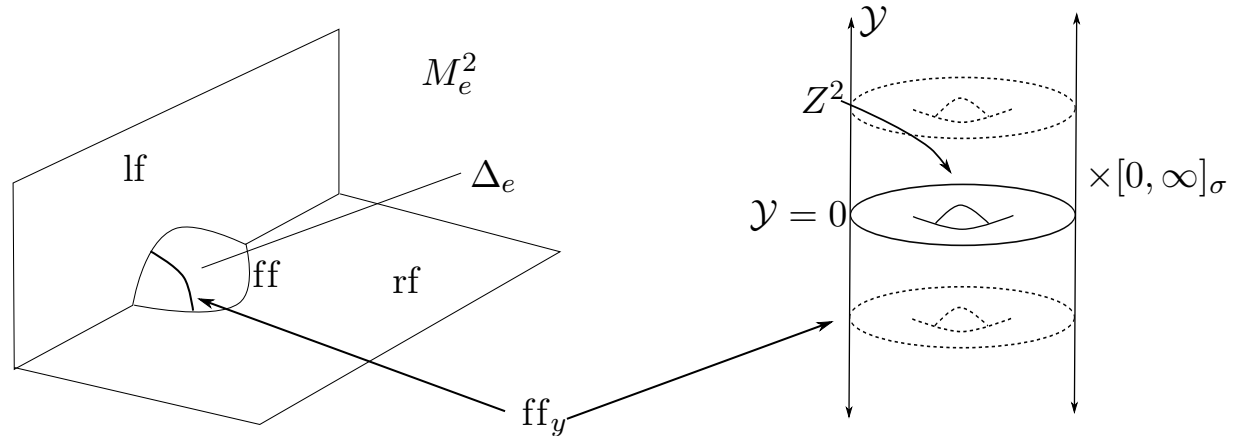

Figure 3.

which is a $b$-map in the sense of [53] and a diffeomorphism from the interior of $M_{\mathrm{e}}^{2}$ to that of $M \times M$. The two boundary hypersurfaces of $M \times M,\{x=0\}$ and $\left\{x^{\prime}=0\right\}$, lift to boundary hypersurfaces of $M_{\mathrm{e}}^{2}$ which we denote by

$$
\text { lf }:=\overline{\beta^{-1}\left(\{x=0\}^{\text {int }}\right)} \quad \text { and } \quad \text { rf }:=\overline{\beta^{-1}\left(\left\{x^{\prime}=0\right\}^{\text {int }}\right)} .
$$

The edge front face, ff, is the radial compactification of the total space of a fiber bundle

$$
Z^{2} \times \mathbb{R}^{b} \times \mathbb{R}_{+} \hookrightarrow \mathrm{ff} \longrightarrow Y
$$

where $b=\operatorname{dim} Y$. This bundle is obtained by pulling-back the tangent bundle of $Y$ and trivial $\mathbb{R}^{+}$ bundle, to the fiber product of two copies of $\partial M$, along the natural projection to $Y$. Choosing local coordinates $(x, y, z)$ as in (2.3), and our fixed bdf $x$, and letting $\left(x, y, z, x^{\prime}, y^{\prime}, z^{\prime}\right)$ denote coordinates on $M \times M$ with $\left(x^{\prime}, y^{\prime}, z^{\prime}\right)$ the same functions as $(x, y, z)$ but on the right factor, the functions

$$
x^{\prime}, \quad \sigma=\frac{x}{x^{\prime}}, \quad \mathcal{Y}=\frac{y-y^{\prime}}{x^{\prime}}, \quad y^{\prime}, \quad z, \quad z^{\prime}
$$

define coordinates near $\mathrm{ff}$ in the set $0 \leq \sigma<\infty$, and in these coordinates $x^{\prime}$ is a boundary defining function for ff, meaning that $\left\{x^{\prime}=0\right\}$ coincides with ff on $\{0 \leq \sigma<\infty\}=M_{\mathrm{e}}^{2} \backslash \mathrm{rf}$, and $x^{\prime}$ has non-vanishing differential on ff. When $x^{\prime}=0, \sigma$ gives coordinates on the $\mathbb{R}_{+}$fiber, $\mathcal{Y}$ on the $\mathbb{R}^{b}$ fiber, and $z, z^{\prime}$ on the $Z^{2}$ fiber. Below we will also use cylindrical coordinates near ff. These have the advantage that they are defined on open neighborhoods of sets in $\mathrm{ff}$ which lie over open sets $V$ in the base $Y$. With $(x, y, z)$ as above, let

$$
\rho_{\mathrm{ff}}=\left(x^{2}+\left(x^{\prime}\right)^{2}+\left|y-y^{\prime}\right|^{2}\right)^{1 / 2}, \quad \phi=\left(\frac{x}{\rho_{\mathrm{ff}}}, \frac{x^{\prime}}{\rho_{\mathrm{ff}}}, \frac{y-y^{\prime}}{\rho_{\mathrm{ff}}}\right),
$$

so $\left(\rho_{\mathrm{ff}}, \phi, y^{\prime}, z, z^{\prime}\right)$ form cylindrical coordinates (in the sense that $|\phi|^{2}=1$ ) near the lift of $V$ to $\mathrm{ff}$ and in the domain of validity of $y, z$.

We now define the calculus of edge pseudodifferential operators with bounds, which is similar to the large calculus of pseudodifferential edge operators defined in [45]. Thus, $\Psi_{\text {e,bnd }}^{m}(M ; \mathcal{S})$ will denote the set of operators $A$ mapping $C_{c}^{\infty}(M ; \mathcal{S})$ to distributional sections $\mathcal{D}^{\prime}(M ; \mathcal{S})$, whose Schwartz kernels have the following structure. Let $\operatorname{End}(\mathcal{S})$ denote the bundle over $M \times M$ whose fiber at $(p, q)$ is $\operatorname{Hom}\left(\mathcal{S}_{q} ; \mathcal{S}_{p}\right)$. The Schwartz kernel of $A, K_{A}$ is a distributional section of the bundle $\operatorname{End}(\mathcal{S})$ over $M \times M$ satisfying that for a section $\phi \in C_{c}^{\infty}(M ; \mathcal{S})$,

$$
A \phi(w)=\int_{M} K_{A}\left(w, w^{\prime}\right) \phi\left(w^{\prime}\right) d \operatorname{Vol}_{g}\left(w^{\prime}\right),
$$


where $d \mathrm{Vol}_{g}$ is the volume form of an incomplete edge metric $g$ asymptotically of the form (2.4). Moreover,

$$
K_{A} \in \mathcal{A}_{a, b, f} I^{m}\left(M_{\mathrm{e}}^{2}, \Delta_{\mathrm{e}} ; \beta^{*} \operatorname{End}(\mathcal{S})\right),
$$

meaning that $K_{A}=K_{1}+K_{2}$ where $\rho_{\mathrm{ff}}^{f} K_{1}$ is in the Hörmander conormal space [37, Chapter 18]

$$
\rho_{\mathrm{ff}}^{f} K_{1} \in I^{m}\left(M_{\mathrm{e}}^{2}, \Delta_{\mathrm{e}} ; \beta^{*} \operatorname{End}(\mathcal{S})\right),
$$

$K_{1}$ is supported near $\Delta_{\mathrm{e}}$, and $K_{2} \in \mathcal{A}\left(M_{\mathrm{e}}^{2} ; \operatorname{End}(\mathcal{S})\right)$, i.e., is smooth in the interior and conormal to the boundary, and satisfies the bounds

$$
K_{2}(p)=O\left(\rho_{\mathrm{lf}}^{a}\right) \quad \text { as } \quad p \rightarrow \mathrm{lf}, \quad K_{2}(p)=O\left(\rho_{\mathrm{rf}}^{b}\right) \quad \text { as } \quad p \rightarrow \mathrm{rf},
$$

where $\rho_{\mathrm{lf}}, \rho_{\mathrm{rf}}$, and $\rho_{\mathrm{ff}}$ are boundary defining functions for lf, $\mathrm{rf}$, and ff respectively. The bound is in the norm on $\operatorname{End}(\mathcal{S})$ over $M \times M$, see [45] for details. Since the bounds $a$ and $b$ in (3.9) will be of some importance, we let

$$
\Psi_{\mathrm{e}}^{m}(M ; \mathcal{S} ; a, b)
$$

denote the subspace of $\Psi_{\mathrm{e}, \mathrm{bnd}}^{m}(M ; \mathcal{S})$ of pseudodifferential edge operators whose Schwartz kernels satisfy (3.9) with bounds $a$ and $b$.

The bounds in (3.9) determine the mapping properties of $A$ on weighted Sobolev spaces. From [45, Theorem 3.25], we have

Theorem 3.6. An element $A \in \Psi_{\mathrm{e}}^{m}(M ; \mathcal{S} ; a, b)$ is bounded as a map

$$
A: x^{\delta} H_{\mathrm{e}}^{k}(M ; \mathcal{S}) \longrightarrow x^{\delta^{\prime}} H^{k-m}(M ; \mathcal{S}),
$$

if and only if

$$
a>\delta^{\prime}-f / 2-1 / 2 \quad \text { and } \quad b>-\delta-f / 2-1 / 2 .
$$

Remark 3.7. In Mazzeo's paper [45] the convention used to describe the weights (orders of vanishing) of the Schwartz kernels of elements in $\Psi_{\mathrm{e}}^{m}$ is slightly different from ours. There one chooses a half-density $\mu$ on $M$ which looks like $\sqrt{d x d y d z}$ near $\partial M$. The choice of $\mu$ gives an isomorphism between the sections of $\mathcal{S}$ and the sections of $\mathcal{S} \otimes \Omega^{1 / 2}(M)$ where $\Omega^{1 / 2}(M)$ is the half-density bundle of $M$ (simply by multiplying by $\mu$ ), and the Schwartz kernel of an edge pseudodifferential operator, $A$, in this context is the section $\kappa_{A}$ of $\operatorname{End}(\mathcal{S}) \otimes \Omega^{1 / 2}\left(M_{\mathrm{e}}^{2}\right)$ with the property that

$$
A(\psi \mu)=\int_{M} \kappa_{A} \psi \mu .
$$

One nice feature of (3.10) is that $\kappa_{A}$ is smooth (away from the diagonal) down to ff. With our convention in (3.8), it is singular of order $-f$ due to the factors of $x$ in the volume form of $g$.

Given an elliptic edge operator $\widetilde{P} \in \operatorname{Diff}_{\mathrm{e}}^{m}(M ; \mathcal{S})$, to construct a parametrix for $\widetilde{P}$ one must study two models for $\widetilde{P}$, the indicial family $I_{y}(\widetilde{P}, \zeta)$ and the normal operator $N(\widetilde{P})_{y}$, in addition to inverting the principal symbol.

First we discuss the indicial operator. For each $y$ in the base $Y$, the indicial family $\zeta \mapsto$ $I_{y}(\widetilde{P}, \zeta)$ is an elliptic operator-valued function on $\mathbb{C}$ obtained by taking the Mellin transform (see [45, Section 2]) of the leading order part of $\widetilde{P}$ in $x$. By (3.2), the leading order part of $\widetilde{P}=x \partial$ is $c\left(\partial_{x}\right)\left(x \partial_{x}+f / 2+\partial_{y}^{Z}\right)$, so taking the Mellin transform and ignoring the $c\left(\partial_{x}\right)$ gives

$$
i \zeta+f / 2+\partial_{y}^{Z}
$$


The meaningful values of $\zeta$ are the indicial roots, which we define to be

$$
\Lambda_{y}=\{i \zeta+f / 2+1 / 2:(3.11) \text { is not invertible }\} .
$$

By definition, (3.11) is invertible as long as $-(i \zeta+f / 2) \notin \sigma\left(\varlimsup_{y}^{Z}\right)$, so Assumption 3.1 implies that

$$
\Lambda_{y} \cap[0,1] \subset\{0,1\} \quad \text { for all } \quad y \in Y .
$$

Remark 3.8. The shift by $f / 2+1 / 2$ in (3.12) comes from the following considerations. We want to understand the mapping properties of $x$ ठ on $L^{2}(M ; \mathcal{S})$ with the natural measure $d \operatorname{Vol}_{g}$ given by the incomplete edge metric $g$. On the other hand, the values of $i \zeta$ for which (3.11) fails to be invertible give information about the mapping properties of $x \gtrsim$ on the Sobolev spaces defined with respect to the $b$-measure

$$
\mu_{b}:=x^{-f-1} d \operatorname{Vol}_{g}
$$

In particular, the Fredholm property in Proposition 3.3 is equivalent to $x$ ठ being a Fredholm map from the space $x^{\delta-f / 2-1 / 2} H_{\mathrm{e}}^{1}\left(M ; \mathcal{S} ; \mu_{b}\right)$ to the space $x^{\delta-f / 2-1 / 2} L_{\mathrm{e}}^{2}\left(M ; \mathcal{S} ; \mu_{b}\right)$, where the Sobolev spaces are now defined with respect to the $b$-measure. Alternatively, as in [3] we could define $\widetilde{P}^{\prime}=x^{-f / 2-1 / 2}(x ð) x^{f / 2+1 / 2}$ take the Mellin transform and use the values of $i \zeta$ as the indicial roots, but we would get the same answer as in (3.12).

Now we discuss the normal operator $N(\widetilde{P})$. Elements of $\mathcal{V}_{\mathrm{e}}$ acting on either factor of $M \times M$ are tangent to ff when lifted to $M_{\mathrm{e}}^{2}$. This implies that, letting $\widetilde{P}$ act on the left on $M \times M$ and lifting to $M_{\mathrm{e}}^{2}, \widetilde{P}$ defines an operator on sections over ff. In fact, we can see explicitly that $\widetilde{P}$ acts on the fibers of ff, so the base $Y$ enters its action only parametrically; that is, for every $y \in Y, \widetilde{P}$ defines an operator

$$
N(\widetilde{P})_{y} \text { acting on the fiber } \mathrm{ff}_{y} \text { over } y \text {. }
$$

To obtain an expression for $N(\widetilde{P})_{y}$ in coordinates, write

$$
\widetilde{P}=\sum_{i+|\alpha|+|\beta| \leq m} a_{i, \alpha, \beta}(x, y, z)\left(x \partial_{x}\right)^{i}\left(x \partial_{y}\right)^{\alpha} \partial_{z}^{\beta}, \quad \text { where } \quad a_{i, \alpha, \beta} \in C^{\infty}(M ; \text { End } \mathcal{S})
$$

and use the projective coordinates in (3.7) to write

$$
N(\widetilde{P})_{y}=\sum_{i+|\alpha|+|\beta| \leq m} a_{i, \alpha, k}(0, y, z)\left(\sigma \partial_{\sigma}\right)^{i}\left(\sigma \partial_{\mathcal{Y}}\right)^{\alpha} \partial_{z}^{\beta} .
$$

The mapping properties of $\widetilde{P}$ are deduced from mapping properties of the $N(\widetilde{P})_{y}$. In particular, to prove Proposition 3.3 we will need Lemma 3.10 below, which shows that the Fourier transform of $N_{y}(x \gtrsim)$ is invertible on certain spaces.

Edge pseudodifferential operators also admit normal operators. Given $A \in \Psi_{\text {e,bds }}^{m}(M ; \mathcal{S})$, the restriction $N(A):=\left.\rho_{\mathrm{ff}}^{f} K_{A}\right|_{\mathrm{ff}}$ is well defined, and in fact

$$
N(A) \in \mathcal{A}_{a, b} I^{m}\left(\mathrm{ff},\left.\Delta_{\mathrm{e}}\right|_{\mathrm{ff}} ;\left.\beta^{*} \operatorname{End}(\mathcal{S})\right|_{\mathrm{ff}}\right),
$$

meaning that $N(A)=\kappa_{1}+\kappa_{2}$ where $\kappa_{1}$ is a distribution on ff conormal to $\Delta_{\mathrm{e}} \cap \mathrm{ff}$ of order $m$ and $\kappa_{2}$ is a smooth function on $\mathrm{ff}^{\text {int }}$ with bounds in (3.9) (with the point $p$ restricted to ff). 
Using (3.2) and the projective coordinates in (3.7), and letting $c_{\nu}$ denote the operator induced by $c\left(\partial_{x}\right)$ on the bundle $\mathcal{S}_{y}$, the restriction of the spin bundle to the fiber over $y$, the normal operator of $x \gtrsim$ satisfies

$$
N(x \partial)=c_{\nu} \cdot\left(\sigma \frac{\partial}{\partial \sigma}+\frac{f}{2}+\check{\partial}_{y^{\prime}}^{Z}\right)+\sigma \partial \mathcal{Y},
$$

where $\partial_{\mathcal{Y}}$ can be written locally in terms of the limiting base metric $h_{y}=\left.g_{Y}\right|_{T_{y} Y}$, i.e., the translation invariant metric on the vector space $T_{y} Y$ defined by $g_{Y}$ in (2.4) as

$$
\partial_{\mathcal{Y}}=\sum_{i, j=1}^{\operatorname{dim} Y} c\left(\partial_{\mathcal{Y}_{i}}\right) h_{y}^{i j} \partial \mathcal{Y}_{j} .
$$

The operator $N(x \gtrsim)$ acts on sections of $\mathcal{S}_{y}$.

The remainder of this subsection consists in establishing the following theorem.

Theorem 3.9. Let $0<\delta<1$. Under Assumption 3.1, there exist left and right parametrices $\widetilde{Q}_{i}$, $i=1,2$ for $x$ ə. Precisely, there are operators $\widetilde{Q}_{i} \in \Psi_{\mathrm{e}, \mathrm{bnd}}^{-1}(M ; \mathcal{S})$, satisfying

$$
\widetilde{Q}_{1} x \partial=\mathrm{Id}-\Pi_{\mathrm{ker}, \delta} \quad \text { and } \quad x \partial \widetilde{Q}_{2}=\mathrm{Id}-\Pi_{\text {coker }, \delta},
$$

where

$$
\widetilde{Q}_{i}: x^{\delta} H_{\mathrm{e}}^{k}(M ; \mathcal{S}) \longrightarrow x^{\delta} H_{\mathrm{e}}^{k+1}(M ; \mathcal{S}), \quad \Pi_{\mathrm{ker}, \delta}, \Pi_{\mathrm{coker}, \delta}: \quad x^{\delta} H_{\mathrm{e}}^{k}(M ; \mathcal{S}) \longrightarrow x^{\delta} H_{\mathrm{e}}^{\infty}(M ; \mathcal{S})
$$

for any $k$. Here $\Pi_{\mathrm{ker}, \delta}\left(\right.$ resp. $\left.\Pi_{\mathrm{coker}, \delta}\right)$ is $x^{\delta} L^{2}(M ; \mathcal{S})$ orthogonal projection onto the kernel (resp. cokernel) of $x$. The Schwartz kernels satisfy the following bounds

$$
\widetilde{Q}_{i} \in \Psi_{\mathrm{e}}^{-1}(M ; \mathcal{S} ; a, b), \quad \Pi_{\mathrm{ker}, \delta}, \Pi_{\mathrm{coker}, \delta} \in \Psi_{\mathrm{e}}^{-\infty}(M ; \mathcal{S} ; a, b),
$$

where $a>\delta-f / 2-1 / 2$ and $b>-\delta-f / 2-1 / 2$. Furthermore, $N\left(\Pi_{\mathrm{ker}, \delta}\right) \equiv 0 \equiv N\left(\Pi_{\mathrm{coker}, \delta}\right)$ so we have

$$
N\left(\widetilde{Q_{1}}\right) N(x ð)=N(\mathrm{Id})=N(x ð) N\left(\widetilde{Q}_{2}\right) .
$$

In particular this establishes that $x \partial: x^{\delta} H_{\mathrm{e}}^{k+1}(M ; \mathcal{S}) \longrightarrow x^{\delta} H_{\mathrm{e}}^{k}(M ; \mathcal{S})$ is Fredholm.

We will see that Theorem 3.9 can be deduced from the work of Mazzeo in [45] and its modifications in [2, 3, 48]. (See also [38], which is closely related to [48], though not directly used in the current work.) In order to see that the results of those papers apply, we must prove that the normal operator $N(x \widetilde{\partial})$ is invertible in a suitable sense. Taking the Fourier transform of $N_{y}(x \partial)$ in $(3.13)$ in the $\mathcal{Y}$ variable gives

$$
L(y, \eta):=\widehat{N_{y}(x \widetilde{\partial})}(\sigma, \eta, z)=c_{\nu} \cdot\left(\sigma \frac{\partial}{\partial \sigma}+\frac{f}{2}+\partial_{y}^{Z}\right)+i \sigma c(\eta)
$$

and for each $y$, one considers the mapping of weighted edge Sobolev spaces defined by picking a positive cutoff function $\phi:[0, \infty)_{\sigma} \longrightarrow \mathbb{R}$ that is 1 near zero and 0 near infinity and letting

$$
\mathcal{H}^{r, \delta, l}:=\left\{u \in \mathcal{D}^{\prime}\left([0, \infty)_{\sigma} \times Z ; \mathcal{S}_{y}\right): \phi u \in \sigma^{\delta} H^{r},(1-\phi) u \in \sigma^{-l} H^{r}\right\}
$$

where, in terms of $k_{y}=\left.g_{N / Y}\right|_{\phi^{*} y}$, the fiber metric on the fiber above $y \in Y$,

$$
H^{r}:=H^{r}\left(\sigma^{f} d \sigma d \mathrm{Vol}_{k_{y}} ; \mathcal{S}_{y}\right)
$$

i.e., it is the standard Sobolev space on $[0, \infty)_{\sigma} \times Z$ whose sections take values in the bundle $\mathcal{S}$ restricted to the boundary over the base point $y$. Consider

$$
L(y, \eta): \mathcal{H}^{r, \delta, l} \longrightarrow \mathcal{H}^{r-1, \delta, l} .
$$


Lemma 3.10. If the fiber operators $\partial_{y}^{Z}$ satisfy Assumption 3.1 for each $y$, then

$$
L(y, \eta): \mathcal{H}^{r, \delta, l} \longrightarrow \mathcal{H}^{r-1, \delta, l}
$$

is invertible for $0<\delta<1$, where $L(y, \eta)$ and $\mathcal{H}^{r, \delta, l}$ are defined in (3.16) and (3.17).

Proof of Lemma 3.10. Given $y \in Y$ and $\eta \in T_{y} Y$ with $\eta \neq 0$, writing $\widehat{\eta}=\eta /|\eta|$, we have $(i c(\widehat{\eta}))^{2}=$ id. Furthermore,

$$
\partial_{y}^{Z} i c(\widehat{\eta})=i c(\widehat{\eta}) \partial_{y}^{Z}
$$

so these operators are simultaneously diagonalizable on $L^{2}\left(Z ; \mathcal{S}_{0, y}, k_{0}\right)$. Thus for each $y$ and $\widehat{\eta}$ we have an orthonormal basis $\left\{\phi_{i, \pm}\right\}_{i=1}^{\infty}$ of $L^{2}\left(Z ; \mathcal{S}_{0, y}, k_{y}\right)$ satisfying

$$
\widetilde{\partial}_{y}^{Z} \phi_{i, \pm}= \pm \mu_{i} \phi_{i, \pm}, \quad i c(\widehat{\eta}) \phi_{i, \pm}= \pm \phi_{i, \pm}, \quad c_{\nu} \phi_{i, \pm}= \pm \phi_{i, \mp} .
$$

Note that the existence of such an orthonormal basis is automatic from the existence of any simultaneous diagonalization $\widetilde{\phi}_{i}$. Indeed, since $c_{\nu}$ is the operator on the bundle $\mathcal{S}_{y}$ induced by $c\left(\partial_{x}\right)$, we have $i c(\widehat{\eta}) c_{\nu} \widetilde{\phi}_{i}=-c_{\nu} \widetilde{\phi}_{i}$, so we can reindex to obtain $\phi_{i, \pm}$ satisfying the two equations on the right in (3.18). But then since $c_{\nu} \widetilde{\partial}_{y}^{Z}=-\widetilde{\partial}_{y}^{Z} c_{\nu}$, the first equation in (3.18) follows automatically. Using the $\phi_{i, \pm}$, we define subspaces of $\mathcal{H}^{r, \delta, l}$ by

$$
W_{i}^{r, \delta, l}=\operatorname{span}\left\{\left(a(\sigma) \phi_{i,+}+b(\sigma) \phi_{i,-}\right): a, b \in \mathcal{H}^{r, \delta, l}(d \sigma)\right\},
$$

where $\mathcal{H}^{r, \delta, l}(d \sigma)$ is defined as in (3.17) in the case that $Z$ is a single point. In particular, for all $\eta$ and $i$,

$$
W_{i}^{r, \delta, l} \subset \mathcal{H}^{r, \delta, l} .
$$

Note that multiplication by $c_{\nu}$ defines a unitary isomorphism of $W_{i}^{r, \delta, l}$. We consider the map $L(y, \eta)$ on each space individually. We claim that

$$
L(y, \eta): W_{i}^{r, \delta, l} \longrightarrow W_{i}^{r-1, \delta, l} \text { is invertible for } 0<\delta<1 .
$$

From (3.16), we compute

$$
-c_{\nu} \cdot L(y, \eta) a(\sigma) \phi_{i, \pm}=\left(\sigma \partial_{\sigma}+\frac{f}{2} \pm \mu\right) a \phi_{i, \pm}-\sigma|\eta| a \phi_{i, \mp}
$$

Thus, writing elements in $W_{i}^{r, \delta, l}$ as vector valued functions $(a, b)^{T}=a \phi_{i,+}+b \phi_{i,-}$, we see that $L(y, \eta)$ indeed maps $W_{i}^{r, \delta, l}$ to $W_{i}^{r-1, \delta, l}$, acting as the matrix

$$
-\left.c_{\nu} L(y, \eta)\right|_{W_{i}}=\sigma \partial_{\sigma}+f / 2+\left(\begin{array}{cc}
\mu & -\sigma|\eta| \\
-\sigma|\eta| & -\mu
\end{array}\right) .
$$

From this, one checks that that the solutions to $L(y, \eta) \phi=0$ can be written using separation of variables as superpositions of sections given, again in terms of the $\phi_{i, \pm}$ by

$$
\mathcal{I}_{\mu, \eta}(\sigma):=\sigma^{-f / 2+1 / 2}\left(\begin{array}{c}
I_{|\mu+1 / 2|}(|\eta| \sigma) \\
I_{|\mu-1 / 2|}(|\eta| \sigma)
\end{array}\right), \quad \mathcal{K}_{\mu, \eta}(\sigma):=\sigma^{-f / 2+1 / 2}\left(\begin{array}{c}
-K_{|\mu+1 / 2|}(|\eta| \sigma) \\
K_{|\mu-1 / 2|}(|\eta| \sigma)
\end{array}\right)
$$

where $I_{\nu}(z)$ and $K_{\nu}(z)$ are the modified Bessel functions [1]. But neither of these lie in $W_{i}^{r, \delta, l}$. Indeed, by the asymptotic formulas [1, equation (9.7)], the sections involving the $\mathcal{I}_{\mu, \eta}$ are not even tempered distributions, as the grow exponentially as $z \rightarrow \infty$. The sections involving 
the $\mathcal{K}_{\mu, \eta}$ are tempered distributions, but since $K_{\nu}(z) \sim z^{-\nu}$ as $z \rightarrow 0$ for $\nu>0$, Assumption 3.1 tells us that $\mu \notin(-1 / 2,1 / 2)$, so $\max \{|\mu-1 / 2|,|\mu+1 / 2|\} \geq 1$. Thus $\mathcal{K}_{\mu, \eta} \notin \mathcal{H}^{r, \delta, l}$ for any $\delta>0$. In other words, Assumption 3.1 implies that

(3.19) is injective if $\delta>0$.

On the other hand, the ordinary differential operator in (3.20) admits an explicit right inverse if $\delta<1$. Specifically, consider the matrix

$$
\begin{aligned}
\mathcal{M}_{\mu,|\eta|}(\sigma, \tilde{\sigma})= & (\sigma \widetilde{\sigma})^{1 / 2}|\eta|\left(\begin{array}{cc}
I_{|\mu+1 / 2|}(|\eta| \sigma) & -K_{|\mu+1 / 2|}(|\eta| \sigma) \\
I_{|\mu-1 / 2|}(|\eta| \sigma) & K_{|\mu-1 / 2|}(|\eta| \sigma)
\end{array}\right) \\
& \times\left(\begin{array}{cc}
-H(\widetilde{\sigma}-\sigma) K_{|\mu-1 / 2|}(|\eta| \tilde{\sigma}) & -H(\widetilde{\sigma}-\sigma) K_{|\mu+1 / 2|}(|\eta| \widetilde{\sigma}) \\
-H(\sigma-\widetilde{\sigma}) I_{|\mu-1 / 2|}(|\eta| \widetilde{\sigma}) & H(\sigma-\widetilde{\sigma}) I_{|\mu+1 / 2|}(|\eta| \widetilde{\sigma})
\end{array}\right) .
\end{aligned}
$$

Then the operator $Q_{y, \mu}$ on $W_{i}^{r-1, \delta, l}$ defined by acting on elements $a(\sigma) \phi_{i,+}+b(\sigma) \phi_{i,-}$ by

$$
Q_{y, \mu}\left(\begin{array}{l}
a \\
b
\end{array}\right):=\sigma^{-f / 2} \int_{0}^{\infty} \mathcal{M}_{\mu,|\eta|}(|\eta|, \sigma, \widetilde{\sigma}) \widetilde{\sigma}^{f / 2-1}\left(\begin{array}{c}
-b(\widetilde{\sigma}) \\
a(\widetilde{\sigma})
\end{array}\right) d \widetilde{\sigma}
$$

satisfies

$$
L(y, \eta) Q_{y, \mu}=\mathrm{id} \quad \text { on } \quad W_{i}^{r, \delta, l} .
$$

(One checks (3.23) using the recurrence relations and Wronskian identity

$$
\begin{aligned}
& I_{\nu}^{\prime}(z)=I_{\nu-1}(z)-\frac{\nu}{z} I_{\nu}(z)=\frac{\nu}{z} I_{\nu}(z)+I_{\nu+1}(z), \\
& K_{\nu}^{\prime}(z)=-K_{\nu-1}(z)-\frac{\nu}{z} K_{\nu}(z)=\frac{\nu}{z} K_{\nu}(z)-K_{\nu+1}(z), \\
& 1 / z=I_{\nu}(z) K_{\nu+1}(z)+I_{\nu+1}(z) K_{\nu}(z),
\end{aligned}
$$

which are equations (9.6.15) and (9.6.26) from [1].) That $Q_{y, \mu}: W_{i}^{r, \delta, l} \longrightarrow W_{i}^{r-1, \delta, l}$ is bounded for $\delta<1$ can be seen using [45], but one can also check it directly using the density of polyhomogeneous functions. Invertibility on each $W_{i}^{r, \delta, l}$ gives invertibility on $\mathcal{H}^{r, \delta, l}$. This proves Lemma 3.10 .

Theorem 3.9 then follows from [45] as explained in [3, Section 2] using the invertibility of the normal operator from Lemma 3.10. In the notation of those papers, one has the numbers

$$
\begin{aligned}
& \bar{\delta}:=\inf \left\{\delta: L(y, \eta): \sigma^{\delta} L^{2}\left(d \sigma d \mathrm{Vol}_{z} ; \mathcal{S}_{y}\right) \longrightarrow L^{2}\left(d \sigma d \mathrm{Vol}_{z} ; \mathcal{S}_{y}\right) \text { is injective for all } y\right\}, \\
& \underline{\delta}:=\sup \left\{\delta: L(y, \eta): \sigma^{\delta} L^{2}\left(d \sigma d \mathrm{Vol}_{z} ; \mathcal{S}_{y}\right) \longrightarrow L^{2}\left(d \sigma d \mathrm{Vol}_{z} ; \mathcal{S}_{y}\right) \text { is surjective for all } y\right\} .
\end{aligned}
$$

By our work above, $\bar{\delta} \leq 0<1 \leq \underline{\delta}$, and thus for the map $x \partial: x^{\delta} H_{\mathrm{e}}^{k} \longrightarrow x^{\delta} H_{\mathrm{e}}^{k}$ with $0<\delta<1$, there exist $\widetilde{Q}_{i}, i=1,2$ satisfying (3.14) for $x$ ə. In particular, by (3.15)

$$
N(x ð)_{y} N\left(\widetilde{Q}_{i}\right)_{y}=N(\mathrm{Id})=\delta_{\beta^{*} \Delta \cap \mathrm{ff}},
$$

where $\beta^{*} \Delta$ is the lift of the interior of the diagonal $\Delta \subset M \times M$ to the blown up space $M^{2}$. Thus in the coordinates $(3.7), \delta_{\beta^{*} \Delta \cap f f}=\delta_{\sigma=1, \mathcal{Y}=0}$, so from (3.21) and (3.22), we can write $N\left(\widetilde{Q}_{i}\right)_{y}$ as follows. For fixed $\eta$ and the basis $\phi_{i, \pm}, i=1,2, \ldots$, from (3.18), let $\Pi(i, \eta)$ denote $L^{2}$ orthogonal projection onto $\phi_{i, \pm}$ and define the vectors

$$
\Pi(\eta, i)=\left(\begin{array}{l}
\pi(\eta, i,+) \\
\pi(\eta, i,-)
\end{array}\right),
$$

where $\pi(\eta, i, \pm)$ is orthogonal projection in $L^{2}\left(Z, \mathcal{S}_{0, y}, k_{y}\right)$ onto $\phi_{i, \pm}$. We thus have

$$
\Pi(\eta, i) \widehat{N\left(\widetilde{Q}_{i}\right)_{y}} \Pi^{*}(\eta, i)=(\widetilde{\sigma} / \sigma)^{f / 2} \widetilde{\sigma}^{-1} \mathcal{M}_{\mu_{i},|\eta|}(\sigma, 1),
$$

where $\Pi^{*}(\eta, i)\left(\begin{array}{l}a \\ b\end{array}\right)=a \phi_{i,+}+b \phi_{i,-}$. 


\subsection{Proof of Theorem 1.1 and the generalized inverse of $ð$}

In this section we will prove Theorem 1.1 and describe the properties of the integral kernel of the generalized inverse of $\nsim$. We start by recalling the statement for the convenience of the reader:

Theorem 3.11. Assume that $\partial$ is a Dirac operator on a spin incomplete edge space $(M, g)$, satisfying Assumption 3.1, then the unbounded operator $\partial$ on $L^{2}(M ; \mathcal{S})$ with core domain $C_{c}^{\infty}(M ; \mathcal{S})$ is essentially self-adjoint. Moreover, letting $\mathcal{D}$ denote the domain of this self-adjoint extension, the map

$$
\text { ð: } \mathcal{D} \longrightarrow L^{2}(M ; \mathcal{S})
$$

is Fredholm.

Proof. The proof of Theorem 1.1 will follow from combining various elements of $[2,3]$. The first and main step is the construction of a left parametrix for the map ə: $\mathcal{D}_{\max } \longrightarrow L^{2}(M ; \mathcal{S})$, where $\mathcal{D}_{\text {max }}$ is the maximal domain defined in (1.6).

Consider $\widetilde{Q}_{1}$ from (3.14) and set $\widetilde{Q}_{1} x=\bar{Q}_{1}$. Then by $(3.14)$

$$
\bar{Q}_{1} \curvearrowright=\mathrm{Id}-\Pi_{\mathrm{ker}, \delta},
$$

where both sides of this equation are thought of as maps of $x^{\delta} L_{\mathrm{e}}^{2}(M ; \mathcal{S})$. We claim that in fact equation $(3.27)$ holds not only on $x^{\delta} L_{\mathrm{e}}^{2}(M ; \mathcal{S})$, but on the maximal domain $\mathcal{D}_{\text {max }}$ defined in (1.6). This follows from [3, Lemma 2.7] as follows. In the notation of that paper, $L=\partial$ and $P=x$ d. Taking (again, in the notation of that paper) $\mathcal{E}(L)$ to be $\mathcal{D}_{\max }$, by [3, Lemma 2.1], $\mathcal{E}^{(\tau)}(L)=\mathcal{E}(L)$. Furthermore, $\mathcal{E}_{\tau}(L)=x^{\tau} L^{2}(M ; \mathcal{S}) \cap \mathcal{D}_{\max }$. Since $\widetilde{Q}_{1}$ maps $x^{\delta} L^{2}(M ; \mathcal{S})$ to $x^{\delta} H_{\mathrm{e}}^{1}$, we have $\operatorname{Id}-\bar{Q}_{1}$ ठ is bounded on $\mathcal{E}_{\tau}$. Futhermore, $x$ ð maps $\mathcal{D}_{\max }$ to $x L^{2}(M ; \mathcal{S}) \subset x^{\delta} L^{2}(M ; \mathcal{S})$, so $\bar{Q}_{1} ð=\widetilde{Q}_{1} x ð$ maps $\mathcal{D}_{\max }$ to $x^{\delta} L^{2}(M ; \mathcal{S})$. Thus [3, Lemma 2.7] applies and (3.27) holds on $\mathcal{D}_{\max }$, as advertised.

Thus Id $=\bar{Q}_{1} \check{\partial}+\Pi_{\mathrm{ker}, \delta}$ on $\mathcal{D}_{\max }$, and since the right hand side is bounded $L^{2}(M ; \mathcal{S})$ to $x^{\delta} L^{2}$, for any $\delta \in(0,1)$, we have

$$
\mathcal{D}_{\max } \subset \bigcap_{\delta<1} x^{\delta} L^{2}(M, \mathcal{S})
$$

in particular for any $\delta<1, \mathcal{D}_{\max } \subset H_{\text {loc }}^{1} \cap x^{\delta} L^{2}(M, \mathcal{S})$ which is a compact subset of $L^{2}(M ; \mathcal{S})$. It then follows from Gil-Mendoza [33] (see [2, Proposition 5.11]) that $\mathcal{D}_{\max } \subset \mathcal{D}_{\text {min }}$, i.e., that $\delta$ is essentially self-adjoint. By a standard argument, e.g., [54, Lemma 4.2], the fact that $\mathcal{D}_{\max }$ includes compactly into $L^{2}(M, \mathcal{S})$ implies that $\partial$ has finite-dimensional kernel and closed range. But the self-adjointness of $\partial$ on $\mathcal{D}$ now implies that $\partial$ has finite-dimensional cokernel, so $\partial$ is self-adjoint and Fredholm.

Thus $\precsim$ admits a generalized inverse $Q: L^{2}(M ; \mathcal{S}) \longrightarrow \mathcal{D}$ satisfying

$$
\text { ऽ } Q=\operatorname{Id}-\pi_{\text {ker }} \quad \text { and } \quad Q=Q^{*},
$$

where $\pi_{\text {ker }}$ is $L^{2}$ orthogonal projection onto the kernel of $\delta$ in $\mathcal{D}$ with respect to the pairing induced by the Hermitian inner product on $\mathcal{S}$. To be precise, if $\left\{\phi_{i}\right\}, i=1, \ldots, N$ is an orthonormal basis for the kernel of $\partial$ on $\mathcal{D}$, then $\pi_{\text {ker }}$ has Schwartz kernel

$$
K_{\pi_{\mathrm{ker}}}\left(w, w^{\prime}\right)=\sum_{i=1}^{N} \phi_{i}(w) \otimes \overline{\phi_{i}\left(w^{\prime}\right)} .
$$


From (3.28), we see that $\pi_{\mathrm{ker}} \in \Psi_{\mathrm{e}}^{-\infty}(M ; \mathcal{S} ; a, b)$. The properties of the integral kernel of $Q$ can be deduced from those of the parametrices $\widetilde{Q}_{i}$ in (3.14). Indeed, setting $\widetilde{Q}=Q x^{-1}$, we see that

$$
\widetilde{Q}(x \widetilde{\partial})=\mathrm{Id}-\pi_{\mathrm{ker}} \quad \text { and } \quad\left(x \widetilde{\mathrm{Q}} \widetilde{Q}=\mathrm{Id}-x \cdot \pi_{\mathrm{ker}} \cdot x^{-1} .\right.
$$

Applying the argument from [45, Section 4], specifically equations (4.24) and (4.25) there, shows that $\widetilde{Q} \in \Psi_{\mathrm{e}}^{-1}(M ; \mathcal{S} ; a, b)$ for the same $a, b$ as in $(3.14)$, and in particular that $N(\widetilde{Q})=N\left(\widetilde{Q}_{i}\right)$. In particular, by Theorem 3.9, we have the bounds

$$
K_{Q}(p)=O\left(\rho_{\mathrm{lf}}^{a}\right) \quad \text { as } \quad p \rightarrow \text { lf } \quad \text { and } \quad K_{Q}(p)=O\left(\rho_{\mathrm{rf}}^{b}\right) \quad \text { as } \quad p \rightarrow \mathrm{rf} \text {, }
$$

where $a>\delta-f / 2-1 / 2$ and $b>-\delta-f / 2+1 / 2,0<\delta<1$, and again the bounds hold for $K_{Q}$ as a section of $\operatorname{End}(\mathcal{S})$ over $M \times M$. Finally, by self-adjointness of $Q$, we have that

$$
K_{Q}\left(w, w^{\prime}\right)=K_{Q}^{*}\left(w^{\prime}, w\right) \quad \text { for all } \quad w, w^{\prime} \in M^{\text {int }} .
$$

By (3.29), the bound at rf, which one approaches in particular if $w$ remains fixed in the interior of $M$ and $w^{\prime}$ goes to the boundary, gives a bound at lf. Thus we obtain the following.

Proposition 3.12. The distributional section $K_{Q}$ of $\operatorname{End}(\mathcal{S})$ over $M \times M$ with the property that $Q \phi=\int_{M} K_{Q}\left(w, w^{\prime}\right) \phi^{\prime}\left(w^{\prime}\right) d \operatorname{Vol}_{g}\left(w^{\prime}\right)$ is conormal at $\Delta_{\mathrm{e}}$, and $\rho_{\mathrm{ff}}^{f-1} K_{Q}$ is smoothly conormal up to ff, where

$$
\left.\rho_{\mathrm{ff}}^{f} K_{Q} x^{-1}\right|_{\mathrm{ff}}=\left.\rho_{\mathrm{ff}}^{f} \widetilde{Q}\right|_{\mathrm{ff}}
$$

satisfies (3.26). Moreover, for coordinates $\left(x, y, z, x^{\prime}, y^{\prime}, z^{\prime}\right)$ on $M \times M$ as in (2.3),

$$
K_{Q}\left(x, y, z, x^{\prime}, y^{\prime}, z^{\prime}\right)=O\left(x^{a}\right), \quad \text { uniformly for } \quad x^{\prime} \geq c>0,
$$

where $a>-\delta-f / 2+1 / 2$ for any $\delta>0$ and $c$ is an arbitrary small positive number.

\section{Boundary values and boundary value projectors}

Recall that $M_{\varepsilon}=\{x \geq \varepsilon\}$ is a smooth manifold with boundary, and $M-M_{\varepsilon}$ is a tubular neighborhood of the singularity. Consider the space of harmonic sections over $M-M_{\varepsilon}$

$$
\mathcal{H}_{\text {loc }, \varepsilon}=\left\{u \in L^{2}\left(M-M_{\varepsilon} ; \mathcal{S}\right): \partial u=0, \exists \widetilde{u} \in \mathcal{D} \text { s.t. } u=\left.\widetilde{u}\right|_{M-M_{\varepsilon}}\right\},
$$

where $\mathcal{D}$ is the domain for $\varnothing$ from Theorem 1.1; in particular, $\mathcal{D} \subset H_{\text {loc }}^{1}$. By the standard restriction theorem for $H^{1}$ sections [60, Proposition 4.5, Chapter 4], any element $u \in \mathcal{H}_{\text {loc, } \varepsilon}$ has boundary values $\left.u\right|_{\partial M_{\varepsilon}} \in H^{1 / 2}\left(\partial M_{\varepsilon}\right)$. We define a domain for $\widetilde{\partial}$ on the cutoff manifold $M_{\varepsilon}$ by

$$
\mathcal{D}_{\varepsilon}:=\left\{u \in H^{1}\left(M_{\varepsilon} ; \mathcal{S}\right):\left.u\right|_{\partial M_{\varepsilon}}=\left.v\right|_{\partial M_{\varepsilon}} \text { for some } v \in \mathcal{H}_{\mathrm{loc}, \varepsilon}\right\} \subset L^{2}\left(M_{\varepsilon} ; \mathcal{S}\right) .
$$

Essentially, $\mathcal{D}_{\varepsilon}$ consists of sections over $M_{\varepsilon}$ whose boundary values correspond with the boundary values of an $L^{2}$ harmonic section over $M-M_{\varepsilon}$. We also have the chirality spaces

$$
\mathcal{D}_{\varepsilon}^{ \pm}=\mathcal{D}_{\varepsilon} \cap L^{2}\left(M_{\varepsilon} ; \mathcal{S}^{ \pm}\right),
$$

where $\mathcal{S}^{ \pm}$are the chirality subbundles of even and odd spinors. In this section we will prove the following.

Theorem 4.1. For $\varepsilon>0$ sufficiently small and $\mathcal{D}_{\varepsilon}$ as in $(4.1)$, the map $\mathrm{\partial}: \mathcal{D}_{\varepsilon} \longrightarrow L^{2}\left(M_{\varepsilon} ; \mathcal{S}\right)$ is Fredholm, and

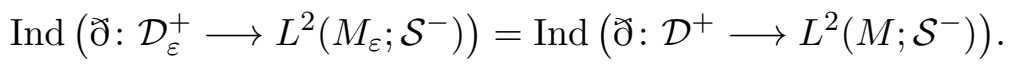

In the process of proving Theorem 4.1, we will construct a family of boundary value projectors $\pi_{\varepsilon}$ which define $\mathcal{D}_{\varepsilon}$ in the sense of Claim 4.2 below, and whose microlocal structure we will use in Section 5 to relate the index of $\partial$ on $M_{\varepsilon}$ with domain $\mathcal{D}_{\varepsilon}$ to the index of $\partial$ on $M_{\varepsilon}$ with the APS boundary condition, see Theorem 5.1. 


\subsection{Boundary value projector for $\mathcal{D}_{\varepsilon}$}

As already mentioned, the main tool for proving Theorem 4.1 and also for proving Theorem 5.1 below will be to express the boundary condition in the definition of $\mathcal{D}_{\varepsilon}$ in (4.1) in terms of a pseudodifferential projection over $\partial M_{\varepsilon}$. We discuss the construction of this projection now.

First we claim that the invertible double construction of [17, Chapter 9] holds in this context in the following form: there exists an incomplete edge manifold $M^{\prime}$ with spinor bundle $\mathcal{S}^{\prime}$ and Dirac operator $ð^{\prime}$, together with an isomorphism

$$
\Phi:\left(M-M_{\varepsilon_{0}}, \mathcal{S}\right) \longrightarrow\left(M^{\prime}-M_{\varepsilon_{0}}^{\prime}, \mathcal{S}^{\prime}\right)
$$

such that, with identifications induced by $\Phi$, the operators $\partial$ and $\partial^{\prime}$ are equal over $M-M_{\varepsilon_{0}}$ $\left(=\Phi^{-1}\left(M^{\prime}-M_{\varepsilon_{0}}^{\prime}\right)\right)$, and finally such that $\partial^{\prime}$ is invertible. In particular, the inverse $Q^{\prime}$ satisfies

$$
Q^{\prime}: \mathcal{D}^{\prime} \longrightarrow L^{2}\left(M^{\prime}\right), \quad \text { } Q^{\prime}=\mathrm{id}=Q^{\prime} \text { ð, }
$$

where $\mathcal{D}^{\prime}$ is the unique self-adjoint domain for $\mathrm{\partial}^{\prime}$ on $M^{\prime}$ with core domain $C_{c}^{\infty}\left(M^{\prime}, \mathcal{S}^{\prime}\right)$. Moreover, $Q^{\prime}$ satisfies all of the properties in Proposition 3.12.

We describe the construction of this "invertible double" for the convenience of the reader, though it is essentially identical to that in [17, Chapter 9], the only difference being that we must introduce a product type boundary while they have one to begin with. Choosing any point $p \in M_{\varepsilon_{0}}$, let $D_{1}, D_{2}$ denote open discs around $p$ with $p \in D_{1} \Subset D_{2}$ and $D_{2} \cap\left(M-M_{\varepsilon_{0}}\right)=\varnothing$. We can identify the annulus $D_{2}-D_{1}$ with $[1,2)_{s} \times \mathbb{S}^{d-1}$ by a diffeomorphism and the metric $g$ is homotopic to a product metric $d s^{2}+|d x|^{2}$ where $x$ is the standard coordinate on $\mathbb{B}^{d-1}$. Furthermore, the connection can be deformed so that the induced Dirac operator $\widetilde{\varpi}^{\prime}$ is of product type on the annulus (see equation (9.4) in [17]). Call the bundle over $N_{1}:=M-D_{1}$ thus obtained $\widetilde{\mathcal{S}}$. Letting $N_{2}:=-N_{1}$, the same incomplete edge space with the opposite orientation, let $M^{\prime}=N_{1} \sqcup N_{2} /\{s=1\}$ and consider the vector bundle $\mathcal{S}^{\prime}$ over $M^{\prime}$ obtained by taking $\widetilde{\mathcal{S}}^{+}$ over $N_{1}$ and $\widetilde{\mathcal{S}}^{-}$over $N_{2}$ and identifying the two bundles over $D_{2}$ using Clifford multiplication by $\partial_{s}$. The resulting Dirac operator, which we still denote by $\check{\partial}^{\prime}$, is seen to be invertible on $M^{\prime}$ by the symmetry and unique continuation argument in Lemma 9.2 of [17].

We will now work on a neighborhood in $M-M_{\varepsilon}$ of $\partial M$ (or equivalently of the singular stratum $Y$ ), so we drop the distinction between $M$ and $M^{\prime}$. Using notation as in (4.2), and given $f \in C^{\infty}\left(\partial M_{\varepsilon} ; \mathcal{S}\right)$, define the harmonic extension

$$
\operatorname{Ext}_{\varepsilon} f(w):=\int_{w^{\prime} \in \partial M_{\varepsilon}} K_{Q^{\prime}}\left(w, w^{\prime}\right) c_{\nu} f\left(w^{\prime}\right) d \operatorname{Vol}_{\partial M_{\varepsilon}}
$$

where $K_{Q^{\prime}}$ is the Schwartz kernel of $Q^{\prime}$ (see (3.8)), and $c_{\nu}=c\left(\partial_{x}\right)$. Since

$$
\varlimsup^{\prime} K_{Q^{\prime}}\left(w, w^{\prime}\right)=0 \quad \text { away from } \quad w=w^{\prime},
$$

$\widetilde{\partial}^{\prime} \operatorname{Ext}_{\varepsilon} f(w)=0$ for $w \notin \partial M_{\varepsilon}$. Recall Green's formula for Dirac operators; specifically, for a smoothly bounded region $\Omega$ with normal vector $\partial_{\nu}$,

$$
\int_{\Omega}(\langle\partial u, v\rangle-\langle u, \widetilde{\partial} v\rangle) d \operatorname{Vol}_{\Omega}=\int_{\partial \Omega}\left\langle c\left(\partial_{\nu}\right) u, v\right\rangle d \operatorname{Vol}_{\partial \Omega} .
$$

Green's formula for sections $u$ satisfying $\partial u \equiv 0$ in $M-M_{\varepsilon}$ gives that for $u \in \mathcal{H}_{\mathrm{loc}, \varepsilon}$,

$$
u(w)=-\int_{\partial M_{\varepsilon}} K_{Q^{\prime}}\left(w, w^{\prime}\right) c_{\nu} u\left(w^{\prime}\right) d \operatorname{Vol}_{\partial M_{\varepsilon}}
$$


provided

$$
\forall u \in \mathcal{H}_{\mathrm{loc}, \varepsilon}, \quad w \in M^{\mathrm{int}}, \quad \lim _{\widetilde{\varepsilon} \rightarrow 0} \int_{\partial M_{\widetilde{\varepsilon}}} K_{Q^{\prime}}\left(w, w^{\prime}\right) c_{\nu} u\left(w^{\prime}\right) d \operatorname{Vol}_{\partial M_{\widetilde{\varepsilon}}}=0
$$

The identity in (4.6) is obtained by integrating by parts in

$$
\int_{M-M_{\varepsilon}} \partial K_{Q^{\prime}}\left(w, w^{\prime}\right) u\left(w^{\prime}\right)-K_{Q^{\prime}}\left(w, w^{\prime}\right) \partial u\left(w^{\prime}\right) d \operatorname{Vol}_{w^{\prime}}
$$

and using (4.4). In fact, as we will see in the proof of Claim 4.2 below, (4.7), and thus (4.6), hold for all $u \in \mathcal{H}_{\text {loc, } \varepsilon \text {. }}$

It follows from (4.3) and (4.6) that, for $\check{\partial} u=0$ satisfying (4.7),

$$
\left.u\right|_{\partial M_{\varepsilon}}(w)=\mathcal{E}_{\varepsilon}\left(\left.u\right|_{\partial M_{\varepsilon}}\right)(w),
$$

where

$$
\mathcal{E}_{\varepsilon}(f)(w):=\lim _{\substack{\widetilde{w} \rightarrow w \\ \widetilde{w} \in M-M_{\varepsilon}}} \operatorname{Ext}_{\varepsilon}(f)(w)
$$

We will show that the $\mathcal{E}_{\varepsilon}$ define the domains $\mathcal{D}_{\varepsilon}$ as follows.

Claim 4.2. The operator $\mathcal{E}_{\varepsilon}$ in $(4.8)$ is a projection operator on $L^{2}\left(\partial M_{\varepsilon}, \mathcal{S}\right)$, and the domain $\mathcal{D}_{\varepsilon}$ in (4.1) is given by

$$
\mathcal{D}_{\varepsilon}=\left\{u \in H^{1}\left(M_{\varepsilon} ; \mathcal{S}\right):\left(\operatorname{Id}-\mathcal{E}_{\varepsilon}\right)\left(\left.u\right|_{\partial M_{\varepsilon}}\right)=0\right\} .
$$

Moreover, there exists $B_{\varepsilon} \in \Psi^{0}\left(\partial M_{\varepsilon} ; \mathcal{S}\right)$ such that

$$
\begin{aligned}
& \mathcal{E}_{\varepsilon}=\frac{1}{2} \operatorname{Id}+B_{\varepsilon} \\
& B_{\varepsilon} f(w)=-\int_{\partial M_{\varepsilon}} K_{Q^{\prime}}\left(w, w^{\prime}\right) c_{\nu} f\left(w^{\prime}\right) d \operatorname{Vol}_{\partial M_{\varepsilon}} \quad \text { for } \quad w \in \partial M_{\varepsilon},
\end{aligned}
$$

and the principal symbol of $\mathcal{E}_{\varepsilon}$ satisfies

$$
\sigma\left(\mathcal{E}_{\varepsilon}\right)=\sigma\left(\pi_{\mathrm{APS}, \varepsilon}\right)
$$

where $\pi_{\mathrm{APS}, \varepsilon}$ is the APS projection defined in (2.16).

Assuming Claim 4.2 for the moment, we prove Theorem 4.1.

Proof of Theorem 4.1 assuming Claim 4.2. The main use of Claim 4.2 in this context (it will be used again in Theorem 5.1 ) is to show that the map

$$
\text { ð: } \mathcal{D}_{\varepsilon} \longrightarrow L^{2}\left(M_{\varepsilon}\right) .
$$

is self-adjoint on $L^{2}\left(M_{\varepsilon} ; \mathcal{S}\right)$ and Fredholm. The Fredholm property follows from the principal symbol equality $(4.11)$, since from [17] any projection in $\Psi^{0}\left(\partial M_{\varepsilon}, \mathcal{S}\right)$ with principal symbol equal to that of the Atiyah-Patodi-Singer boundary projection defines a Fredholm problem. To see that it is self-adjoint, note that from (4.5) the adjoint boundary condition is

$$
\mathcal{D}_{\varepsilon}^{*}=\left\{\phi:\left\langle g,\left.c_{\nu} \phi\right|_{\partial M_{\varepsilon}}\right\rangle_{\partial M_{\varepsilon}}=0 \text { for all } g \text { with }\left(\mathrm{Id}-\mathcal{E}_{\varepsilon}\right) g=0\right\} .
$$


Again by (4.5), for any $v \in \mathcal{H}_{\mathrm{loc}, \varepsilon},\left.v\right|_{\partial M_{\varepsilon}} \in \mathcal{D}_{\varepsilon}^{*}$. Thus $\mathcal{D}_{\varepsilon} \subset \mathcal{D}_{\varepsilon}^{*}$, and it remains to show that $\mathcal{D}_{\varepsilon}^{*} \subset \mathcal{D}_{\varepsilon}$. Let $\phi \in \mathcal{D}_{\varepsilon}^{*}$, and set $f:=\left.\phi\right|_{\partial M_{\varepsilon}}$. We want to show that $\left(I-\mathcal{E}_{\varepsilon}\right) f=0$, or equivalently

$$
\left\langle\left(I-\mathcal{E}_{\varepsilon}\right) f, g\right\rangle_{\partial M_{\varepsilon}}=0 \quad \forall g \quad \Longleftrightarrow \quad\left\langle f,\left(I-\mathcal{E}_{\varepsilon}^{*}\right) g\right\rangle_{\partial M_{\varepsilon}}=0 \quad \forall g .
$$

Since $\left\langle f, c_{\nu} g\right\rangle=-\left\langle c_{\nu} f, g\right\rangle$, by (4.5) we have $\left\langle f, c_{\nu} g\right\rangle=0$ for every $g \in \operatorname{Ran} \mathcal{E}$, and thus (4.13) will hold if $\left(I-\mathcal{E}_{\varepsilon}^{*}\right) g \in \operatorname{Ran} c_{\nu} \mathcal{E}$. In fact, we claim that

$$
I-\mathcal{E}_{\varepsilon}^{*}=-c_{\nu} \mathcal{E} c_{\nu}
$$

To see that his holds, note that by Claim 4.2 and self-adjointness of $Q^{\prime}$, specifically (3.29), $B_{\varepsilon}^{*}=c_{\nu} B_{\varepsilon} c_{\nu}$, so

$$
I-\mathcal{E}_{\varepsilon}^{*}=I-\left(\frac{1}{2}+B_{\varepsilon}\right)^{*}=\frac{1}{2}-B_{\varepsilon}^{*}=-c_{\nu}\left(\frac{1}{2}+B_{\varepsilon}\right) c_{\nu}=-c_{\nu} \mathcal{E}_{\varepsilon} c_{\nu}
$$

which proves self-adjointness.

Now that we know that (4.12) is self-adjoint, we proceed as follows. We claim that for $\varepsilon>0$ sufficiently small, the map

$$
\begin{aligned}
\operatorname{ker}\left(ð: \mathcal{D} \longrightarrow L^{2}(M ; \mathcal{S})\right) & \longrightarrow \operatorname{ker}\left(\widetilde{\partial}: \mathcal{D}_{\varepsilon} \longrightarrow L^{2}\left(M_{\varepsilon} ; \mathcal{S}\right)\right), \\
\widetilde{\phi} & \longmapsto \phi=\left.\widetilde{\phi}\right|_{M_{\varepsilon}}
\end{aligned}
$$

is well defined and an isomorphism. It is well defined since by definition any section $\widetilde{\phi} \in$ $\operatorname{ker}\left(\check{\partial}: \mathcal{D} \longrightarrow L^{2}(M ; \mathcal{S})\right)$ satisfies that $\phi=\left.\widetilde{\phi}\right|_{M_{\varepsilon}} \in \mathcal{D}_{\varepsilon}$. It is injective by unique continuation. For surjectivity, note that for any element $\phi \in \operatorname{ker}\left(ð: \mathcal{D}_{\varepsilon} \longrightarrow L^{2}\left(M_{\varepsilon} ; \mathcal{S}\right)\right.$ ), by definition there is a $u \in \mathcal{H}_{\text {loc }, \varepsilon}$ such that $\left.u\right|_{\partial M_{\varepsilon}}=\left.\phi\right|_{\partial M_{\varepsilon}}$. It follows that

$$
\widetilde{\phi}(w):= \begin{cases}\phi(w) & \text { for } w \in M_{\varepsilon}, \\ u(w) & \text { for } w \in M-M_{\varepsilon}\end{cases}
$$

is in $H^{1}$ and satisfies $\widetilde{\partial} \tilde{\phi}=0$ on all of $M$, i.e., $\widetilde{\phi} \in \operatorname{ker}\left(\widetilde{\partial}: \mathcal{D} \longrightarrow L^{2}(M ; \mathcal{S})\right)$. Since the full operator $\partial$ on $\mathcal{D}$ is self-adjoint, and since the operator in (4.12) is also, the cokernels of both maps are equal to the respective kernels. Restricting ठे to a map from sections of $\mathcal{S}^{+}$to sections of $\mathcal{S}^{-}$gives the theorem.

This completes the proof.

Thus to prove Theorem 4.1 it remains to prove Claim 4.2.

Proof of Claim 4.2. We begin by proving (4.10). It is a standard fact (see [61, Section 7.11]) that

$$
\mathcal{E}_{\varepsilon} \in \Psi^{0}\left(\partial M_{\varepsilon} ; \mathcal{S}\right)
$$

Obviously,

$$
\mathcal{E}_{\varepsilon}=A+B_{\varepsilon}
$$

where

$$
\begin{aligned}
& B_{\varepsilon} f(w)=-\int_{\partial M_{\varepsilon}} K_{Q^{\prime}}\left(w, w^{\prime}\right) c_{\nu} f\left(w^{\prime}\right) d \operatorname{Vol}_{\partial M_{\varepsilon}} \quad \text { for } \quad w \in \partial M_{\varepsilon}, \\
& \operatorname{supp} A \subset \operatorname{diag}\left(\partial M_{\varepsilon} \times \partial M_{\varepsilon}\right),
\end{aligned}
$$


where the last containment refers to the Schwartz kernel of $A$. We claim that

$$
A=\frac{1}{2} \text { id } \quad \text { and } \quad B_{\varepsilon} \in \Psi^{0}\left(\partial M_{\varepsilon} ; \mathcal{S}\right)
$$

Using that $Q^{\prime}$ has principal symbol $\sigma\left(Q^{\prime}\right)=\sigma(ð)^{-1}$ we can write $Q^{\prime}$ in local coordinates $w$ as

$$
Q^{\prime}=\frac{1}{(2 \pi)^{n}} \int_{\mathbb{R}^{n}} e^{-(w-\widetilde{w}) \cdot \xi} a(w, \widetilde{w}, \xi) d \xi \quad \text { locally, }
$$

where

$$
a(w, \widetilde{w}, \xi)=|\xi|_{g(w)}^{-2} i c(\xi)+O(1) \quad \text { for } \quad|\xi| \geq c>0 .
$$

Given a bump function $\chi$ supported near $w_{0} \in \partial M_{\varepsilon}$, let $Q_{\chi}^{\prime}:=\chi Q^{\prime} \chi$ and define the distributions

$$
K_{Q_{\chi}^{\prime}}=K_{1}+K_{2},
$$

where

$$
K_{1}=\mathcal{F}_{\xi}^{-1}\left(|\xi|_{g(w)}^{-2} i c(\xi)\right) \mathcal{F}_{\widetilde{x}}
$$

where, as in (3.8), $K_{Q_{\chi}^{\prime}}$ denotes the Schwartz kernel of $Q_{\chi}^{\prime}$. The distribution $K_{2}$ is that of a pseudodifferential operator of order -2 on $M$, and it follows from the theory of homogeneous distributions (see [61, Chapter 7]) that the distribution $K_{2}$ restricts to $\partial M_{\varepsilon}$ to be the Schwartz kernel of a pseudodifferential operator of order -1 . The distribution $K_{1}$ is that of a pseudodifferential operator on $M$ of order -1 . It is smooth in $\widetilde{x}$ with values in homogeneous distributions in $x-\widetilde{x}$ of order $-n+1$, and it follows that the restriction of the Schwartz kernel $K_{1}\left(w, w^{\prime}\right)$ to $\partial M_{\varepsilon}$ gives a pseudodifferential operator of order zero. Letting $B_{\varepsilon}$ in $(4.14)$ be the operator defined by the restriction of $K_{1}$ to $\partial M_{\varepsilon}$, we have that $B_{\varepsilon}$ is in $\Psi^{0}\left(\partial M_{\varepsilon} ; \mathcal{S}\right)$ and it remains to calculate $A$. Choosing coordinates of the form $w=\left(x, x^{\prime}\right)$ and $\widetilde{w}=\left(\widetilde{x}, \widetilde{x}^{\prime}\right)$ of the form in (2.14) and such that at the fixed value $w_{0}=\left(\varepsilon, x_{0}^{\prime}\right) \in \partial M_{\varepsilon}$ the metric satisfies $g(x)=\mathrm{id}$, it follows (see [4]) that the Schwartz kernel of $B$ in (4.15) satisfies

$$
B\left(x_{0}, \widetilde{x}\right)=-\frac{1}{\omega_{n-1}} \frac{c\left(x_{0}\right)-c(\widetilde{x})}{\left|x_{0}-\widetilde{x}\right|^{n}}+O\left(\left|x_{0}-\widetilde{x}\right|^{2-n}\right),
$$

where $\omega_{n-1}$ is the volume of the unit sphere $\mathbb{S}^{n-1}$. If we let $\widetilde{B}\left(x^{\prime}, \widetilde{x}^{\prime}\right)=B\left(0, x^{\prime}, 0, \widetilde{x}^{\prime}\right)$, then near $x_{0}$

$$
\begin{aligned}
\operatorname{Ext}_{\varepsilon} f\left(\delta, x^{\prime}\right) & =-\frac{1}{\omega_{n-1}} \int\left(\frac{c\left(\left(\delta, x^{\prime}\right)\right)-c\left(\left(0, \widetilde{x}^{\prime}\right)\right)}{\left|\left(\delta, x^{\prime}\right)-\left(0, \widetilde{x}^{\prime}\right)\right|^{n}}\right) c_{\nu} f\left(\widetilde{x}^{\prime}\right) d \widetilde{x}^{\prime} \\
& =-\frac{1}{\omega_{n-1}} \int\left(\frac{\delta c_{\nu}}{\left|\left(\delta, x^{\prime}\right)-\left(0, \widetilde{x}^{\prime}\right)\right|^{n}}+\frac{c\left(\left(0, x^{\prime}\right)\right)-c\left(\left(0, \widetilde{x}^{\prime}\right)\right)}{\left|\left(\delta, x^{\prime}\right)-\left(0, \widetilde{x}^{\prime}\right)\right|^{n}}\right) c_{\nu} f\left(\widetilde{x}^{\prime}\right) d \widetilde{x}^{\prime} \\
& \rightarrow \frac{1}{2} f\left(x^{\prime}\right)+\int \widetilde{B}\left(x^{\prime}, y^{\prime}\right) c_{\nu} f\left(y^{\prime}\right) d y^{\prime} \quad \text { as } \quad \delta \rightarrow 0 .
\end{aligned}
$$

This proves that $A=1 / 2$.

The principal symbol of $\mathcal{E}_{\varepsilon}$ (again see [61, Section 7.11]) is given by the integral

$$
\begin{aligned}
\sigma(\mathcal{E})\left(x^{\prime}, \xi^{\prime}\right) & =\frac{-1}{2 \pi} \lim _{x \rightarrow \varepsilon^{-}} \int_{\mathbb{R}} e^{i(x-\varepsilon) \xi} \frac{1}{\left|\left(\xi, \xi^{\prime}\right)\right|_{g}^{2}}\left(i c\left(\xi \partial_{x}\right)+i c\left(\xi^{\prime} \cdot \partial_{x^{\prime}}\right)\right) c\left(\partial_{x}\right) d \xi \\
& =\frac{-1}{2 \pi} \lim _{x \rightarrow \varepsilon^{-}}\left(\int_{\mathbb{R}} e^{i(x-\varepsilon) \xi} \frac{\xi}{\left|\left(\xi, \xi^{\prime}\right)\right|_{g}^{2}} d \xi\right) i c\left(\partial_{x}\right)^{2}-\frac{1}{2} i c\left(\widehat{\xi}^{\prime} \cdot \partial_{x^{\prime}}\right) c\left(\partial_{x}\right) \\
& =\frac{-1}{2 \pi}\left(-\frac{2 \pi i}{2}\right) i c\left(\partial_{x}\right)^{2}-\frac{1}{2}\left(-i c\left(\partial_{x} c\left(\widehat{\xi}^{\prime} \cdot \partial_{x^{\prime}}\right)\right)\right. \\
& \left.=\frac{1}{2}-\frac{1}{2}\left(-i c\left(\partial_{x}\right) c\left(\widehat{\xi}^{\prime} \cdot \partial_{x^{\prime}}\right)\right)\right)
\end{aligned}
$$


where in the third line we used the residue theorem. Now recall that the term $\left.-i c\left(\partial_{x}\right) c\left(\widehat{\xi}^{\prime} \cdot \partial_{x^{\prime}}\right)\right)$ is precisely the endomorphism appearing in (2.15), so

$$
\sigma(\mathcal{E})\left(x^{\prime}, \xi^{\prime}\right)=\frac{1}{2}-\frac{1}{2}\left(\pi_{\varepsilon,+, \widehat{\xi}^{\prime}}\left(x^{\prime}\right)-\pi_{\varepsilon,-, \widehat{\xi}^{\prime}}\left(x^{\prime}\right)\right)=\pi_{\varepsilon,-, \widehat{\xi}^{\prime}}\left(x^{\prime}\right),
$$

where the projections are those in (2.15). Thus, Theorem 2.3 implies the desired formula for the principal symbol of $\mathcal{E}_{\varepsilon},(4.11)$.

To finish the claim, we must show the equivalence of domains in (4.9). We first show that for any $u \in \mathcal{H}_{\text {loc }, \varepsilon}$, the formula in (4.6) holds. This will show that any $f \in H^{1 / 2}\left(\partial M_{\varepsilon} ; \mathcal{S}\right)$ with $f=\left.u\right|_{\partial M_{\varepsilon}}$ for some $u \in \mathcal{H}_{\text {loc, } \varepsilon}$ satisfies $\left(\operatorname{Id}-\mathcal{E}_{\varepsilon}\right) f=0$, i.e., that

$$
\mathcal{D}_{\varepsilon} \subset\left\{u \in H^{1}\left(M_{\varepsilon} ; \mathcal{S}\right):\left(\operatorname{Id}-\mathcal{E}_{\varepsilon}\right)\left(\left.u\right|_{\partial M_{\varepsilon}}\right)=0\right\} .
$$

Thus we must show that (4.7) holds for $u \in \mathcal{H}_{\mathrm{loc}, \varepsilon}$. For such $u$, we claim that for some $\delta>0$, as $\varepsilon \rightarrow 0$

$$
\int_{\partial M_{\varepsilon}}\|u\|^{2} d \operatorname{Vol}_{\partial M_{\varepsilon}}=O\left(\varepsilon^{-f-\delta}\right)
$$

To see this, note first that $u \in x^{1-\delta} H_{\mathrm{e}}^{1}\left(M-M_{\varepsilon}, \mathcal{S}\right)$ for every $\delta>0$, which follows since $u$ has an extension to a section in $\mathcal{D}_{\max } \subset H_{\text {loc }}^{1} \cap_{\delta>0} x^{1-\delta} L^{2}(M ; \mathcal{S})$. In particular, $x^{\delta+f / 2} u \in$ $H^{1}(M, d x d y d z)$, the standard Sobolev space of order 1 on the manifold with boundary $M$. Using the restriction theorem [60, Proposition 4.5, Chapter 4], $x^{\delta+f / 2} u=\varepsilon^{\delta+f / 2} u \in H^{1 / 2}(\partial M)$ uniformly in $\varepsilon$, so (4.16) holds. Thus, for fixed $w \in M-M_{\varepsilon}$, writing $d \operatorname{Vol}_{g}=x^{f} a d x d y d z$ for some $a=a(x, y, z)$ with $a(0, y, z) \neq 0$, we can use the bound for $K_{Q^{\prime}}$ in (3.30) with $x^{\prime}$ fixed and $x=\varepsilon$ to conclude

$$
\begin{aligned}
& \left(\int_{\partial M_{\varepsilon}} K_{Q^{\prime}}\left(w, w^{\prime}\right) c_{\nu} u\left(w^{\prime}\right) d \operatorname{Vol}_{\partial M_{\varepsilon}}\right)^{2}=\left(\int_{\partial M_{\varepsilon}} K_{Q^{\prime}}\left(w, w^{\prime}\right) c_{\nu} u\left(w^{\prime}\right) \varepsilon^{f} a d y^{\prime} d z^{\prime}\right)^{2} \\
& \quad \leq \varepsilon^{2 f}\left(\int_{\partial M_{\varepsilon}}\left\|K_{Q^{\prime}}\left(w, w^{\prime}\right)\right\|^{2} a d y^{\prime} d z^{\prime}\right)\left(\int_{\partial M_{\varepsilon}}\|u\|^{2} a d y^{\prime} d z^{\prime}\right) \\
& \quad=\varepsilon^{2 f} o\left(\varepsilon^{-2 \delta-f+1}\right) o\left(\varepsilon^{-f-2 \delta}\right) \quad \text { for all } \delta>0 \\
& =o\left(\varepsilon^{-4 \delta+1}\right) \rightarrow 0 \quad \text { as } \varepsilon \rightarrow 0 .
\end{aligned}
$$

To prove the other direction of containment in (4.9), we need to know that for $f \in H^{1 / 2}\left(\partial M_{\varepsilon}\right)$ satisfying $\left(\operatorname{Id}-\mathcal{E}_{\varepsilon}\right) f=0$, the section $u:=\left.\operatorname{Ext}_{\varepsilon} f\right|_{M-M_{\varepsilon}} \in \mathcal{H}_{\mathrm{loc}, \varepsilon}$, where Ext $\operatorname{Ex}_{\varepsilon}$ is the extension operator in (4.3). This is true since for any $H^{1 / 2}$ section $h$ over $\partial M_{\varepsilon}$, there is an $H^{1}$ extension $v$ to the manifold $M^{\prime}$ defined above, that can be taken with support away from the singular locus. If $1_{M_{\varepsilon}^{\prime}}$ is the indicator function of $M_{\varepsilon}^{\prime}$, then $\widetilde{\partial}^{\prime}\left(\operatorname{Ext}_{\varepsilon} f+1_{M_{\varepsilon}^{\prime}} v\right)=\delta_{\partial M_{\varepsilon}}(f+h)+1_{M_{\varepsilon}^{\prime}} \mathrm{\partial}^{\prime} v$. Taking $h$ to cancel $f$ gives that $\widetilde{\partial}^{\prime}\left(\operatorname{Ext}_{\varepsilon} f+1_{M_{\varepsilon}^{\prime}} v\right) \in L^{2}\left(M^{\prime} ; \mathcal{S}\right)$. Since $1_{M_{\varepsilon}^{\prime}} v$ is an extendible $H^{1}$ distribution on $M_{\varepsilon}^{\prime}$ near $\partial M_{\varepsilon}$, Ext $\left.f\right|_{M-M_{\varepsilon}}$ is an extendible $H_{\mathrm{loc}}^{1}$ distribution on $M-M_{\varepsilon}$ near $\partial M_{\varepsilon}$. This completes the proof of Claim 4.2.

\section{Equivalence of indices}

In the previous section we have shown

$$
\text { Ind }\left(ð: \mathcal{D}_{\varepsilon}^{+} \longrightarrow L^{2}\left(M_{\varepsilon} ; \mathcal{S}^{-}\right)\right)=\operatorname{Ind}\left(ð: \mathcal{D}^{+} \longrightarrow L^{2}\left(M ; \mathcal{S}^{-}\right)\right) \text {, }
$$

in this section we will prove the following. 
Theorem 5.1. Let $\pi_{\mathrm{APS}, \varepsilon}$ denote the APS projector from (2.17). Then for $\varepsilon>0$ sufficiently small,

$$
\text { Ind }\left(ð: \mathcal{D}_{\mathrm{APS}, \varepsilon}^{+} \longrightarrow L^{2}\left(M_{\varepsilon} ; \mathcal{S}^{-}\right)\right)=\operatorname{Ind}\left(ð: \mathcal{D}_{\varepsilon}^{+} \longrightarrow L^{2}\left(M_{\varepsilon} ; \mathcal{S}^{-}\right)\right) \text {, }
$$

where $\mathcal{D}_{\varepsilon}$ is the domain in (4.9) and $\mathcal{D}_{\mathrm{APS}, \varepsilon}$ is the domain in $(2.17)$.

The main tool for proving Theorem 5.1 is the following theorem from [17]. We define the 'pseudodifferential Grassmanians'

$$
\operatorname{Gr}_{\mathrm{APS}, \varepsilon}=\left\{\pi \in \Psi^{0}\left(\partial M_{\varepsilon} ; \mathcal{S}\right): \pi^{2}=\pi \text { and } \sigma(\pi)=\sigma\left(\pi_{\mathrm{APS}, \varepsilon}\right)\right\} .
$$

We endow $\mathrm{Gr}_{\mathrm{APS}, \varepsilon}$ with the norm topology. If $\pi \in \mathrm{Gr}_{\mathrm{APS}, \varepsilon}$, then defining the domain $\mathcal{D}_{\pi, \varepsilon}=$ $\left\{u \in H^{1}\left(M_{\varepsilon} ; \mathcal{S}\right):(\mathrm{Id}-\pi)\left(\left.u\right|_{\partial M_{\varepsilon}}\right)=0\right\}$, the map

$$
\text { ə: } \mathcal{D}_{\pi, \varepsilon} \longrightarrow L^{2}\left(M_{\varepsilon} ; \mathcal{S}\right)
$$

is Fredholm. The following follows from [17, Theorem 20.8] and [17, Theorem 15.12]

Theorem 5.2. If $\pi_{i} \in \mathrm{Gr}_{\mathrm{APS}, \varepsilon}, i=1,2$ lie in the same connected component of $\mathrm{Gr}_{\mathrm{APS}, \varepsilon}$ then the elliptic boundary problems

$$
\text { ə: } \mathcal{D}_{\pi_{i}, \varepsilon}^{+} \longrightarrow L^{2}\left(M_{\varepsilon}, \mathcal{S}^{-}\right)
$$

have equal indices.

To apply Theorem 5.2 in our case, we will study the two families of boundary values projectors $\pi_{\mathrm{APS}, \varepsilon}$ and $\mathcal{E}_{\varepsilon}$ using the adiabatic calculus of Mazzeo and Melrose [46].

\subsection{Review of the adiabatic calculus}

Consider a fiber bundle $Z \hookrightarrow \widetilde{X} \stackrel{\pi}{\longrightarrow} Y$. The adiabatic double space $\widetilde{X}_{\text {ad }}^{2}$ is formed by radial blow up of $\widetilde{X}^{2} \times\left[0, \varepsilon_{0}\right)_{\varepsilon}$ along the fiber diagonal, $\operatorname{diag}_{\mathrm{fib}}(\widetilde{X})$ (see (3.6)) at $\varepsilon=0$. That is,

$$
\widetilde{X}_{\mathrm{ad}}^{2}=\left[\widetilde{X}^{2} \times\left[0, \varepsilon_{0}\right)_{\varepsilon} ; \operatorname{diag}_{\mathrm{fib}}(\widetilde{X}) \times\{\varepsilon=0\}\right] .
$$

Thus, $\widetilde{X}_{\text {ad }}^{2}$ is a manifold with corners with two boundary hypersurfaces: the lift of $\{\varepsilon=0\}$, which we continue to denote by $\{\varepsilon=0\}$, and the one introduced by the blowup, which we call ff. Similar to the edge front face above, ff is a bundle over $Y$ whose fibers are isomorphic to $Z^{2} \times \mathbb{R}^{b}$ where $b=\operatorname{dim} Y$, and in fact this is the fiber product of $\pi^{*} T^{*} Y$ and $\tilde{X}$.

We define $\mathrm{ff}_{y}$ to be the fiber of ff lying above $y$.

The adiabatic vector fields on the fibration $\widetilde{X}$ are families of vector fields $V_{\varepsilon}$ parametrized smoothly in $\varepsilon \in\left[0, \varepsilon_{0}\right)$, such that $V_{0}$ is a vertical vector field, i.e., a section of $T \widetilde{X} / Y$. Locally these are $C^{\infty}\left(\widetilde{X} \times\left[0, \varepsilon_{0}\right)_{\varepsilon}\right)$ linear combinations of the vector fields

$$
\partial_{z}, \quad \varepsilon \partial_{y}
$$

Such families of vector fields are in fact sections of a vector bundle

$$
T_{\mathrm{ad}}(\partial M) \longrightarrow \partial M \times\left[0, \varepsilon_{0}\right)_{\varepsilon} .
$$

We will now define adiabatic differential operators on sections of $\mathcal{S}$. The space of $m^{\text {th }}$ order adiabatic differential operators $\operatorname{Diff}_{\text {ad }}^{m}(\widetilde{X} ; \mathcal{S})$ is the space of differential operators obtained by 


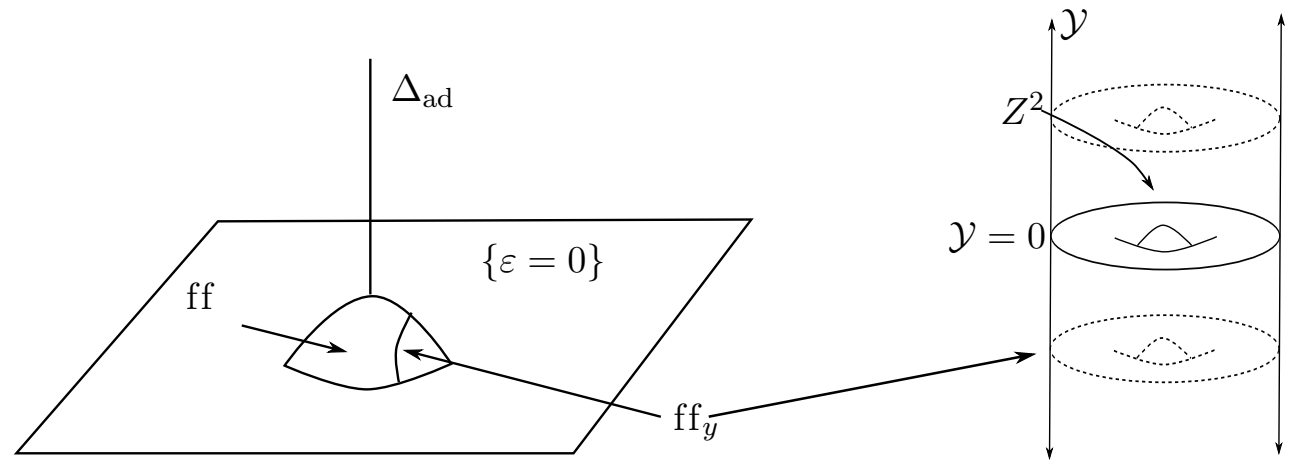

Figure 4. The adiabatic double space.

taking $C^{\infty}(\widetilde{X} ; \operatorname{End}(\mathcal{S}))$ combinations of powers (up to order $m$ ) of adiabatic vector fields. An adiabatic differential operator $P$ admits a normal operator $N(P)$, obtained by letting $P$ act on $\widetilde{X} \times \widetilde{X} \times\left[0, \varepsilon_{0}\right)_{\varepsilon}$, pulling back $P$ to $\widetilde{X}_{\text {ad }}^{2}$, and restricing it to ff. The normal operator acts tangentially along the fibers of ff over $Y$, and $N(P)_{y}$ will denote the operator on sections of $\mathcal{S}$ restricted to over $\mathrm{ff}_{y}$. More concretely, if $P$ is an adiabatic operator of order $m$, then near a point $y_{0}$ in $Y$, we can write

$$
P=\sum_{|\alpha|+|\beta| \leq m} a_{\alpha, \beta}(z, y, \varepsilon) \partial_{z}^{\alpha}\left(\varepsilon \partial_{y}\right)^{\beta}
$$

for $y$ near $y_{0}$, where $a_{\alpha, \beta}(z, y, \varepsilon)$ is a smooth family of endomorphisms of $\mathcal{S}$. The normal operator is given by

$$
N(P)_{y_{0}}=\sum_{|\alpha|+|\beta| \leq m} a_{\alpha, \beta}\left(z, y_{0}, 0\right) \partial_{z}^{\alpha} \partial_{\mathcal{Y}}^{\beta}
$$

where $\mathcal{Y}$ are coordinates on $\mathbb{R}^{b}$. Thus $N(P)_{y_{0}}$ is a differential operator on $Z_{y_{0}} \times T_{y_{0}} Y$ that is constant coefficient in the $T Y$ direction.

Returning to the case that $\widetilde{X}=\partial M$ for $M$ a compact manifold with boundary, we take a collar neighborhood $\mathcal{U} \simeq \partial M \times\left[0, \varepsilon_{0}\right)_{x}$ as in (3.1), and treating the boundary defining function, $x$, as the parameter $\varepsilon$ in the previous paragraph, identify the adiabatic double space $(\partial M)_{\mathrm{ad}}^{2}$ with a blow up of $\left\{x=x^{\prime}\right\} \subset \mathcal{U} \times \mathcal{U}$.

Lemma 5.3. The tangential operator $\widetilde{\widetilde{\partial}}_{\varepsilon}$ defined in $(2.13)$ lies in $\operatorname{Diff}_{\text {ad }}^{m}(\partial M ; \mathcal{S})$. The normal operator of $\widetilde{\widetilde{\partial}}_{\varepsilon}$ satisfies

$$
N\left(\widetilde{\widetilde{\partial}}_{\varepsilon}\right)_{y}=\widetilde{\partial}_{y}^{Z}-c_{\nu} \partial_{\mathcal{Y}}
$$

where $\mathrm{\partial}_{y}^{Z}$ is as in (3.3), and $\partial_{\mathcal{Y}}$ is the standard Dirac operator on $T_{y} Y$.

Proof. This follows from equation (3.13) above.

The space of adiabatic pseudodifferential operators with bounds on $\widetilde{X}$ of order $m$ acting on sections of $\mathcal{S}$, denoted $\Psi_{\text {ad,bnd }}^{m}(\widetilde{X} ; \mathcal{S})$, is the space of families of pseudodifferential operators $\left\{A_{\varepsilon}\right\}_{0<\varepsilon<\varepsilon_{0}}$, where $A_{\varepsilon}$ is a (standard) $\Psi$ of order $m$ for each $\varepsilon$, and whose integral kernel of $A_{\varepsilon}$ is conormal to the lifted diagonal $\Delta_{\text {ad }}:=\overline{\operatorname{diag}_{\tilde{X}} \times\left(0, \varepsilon_{0}\right)_{\varepsilon}}$, smoothly up to ff. To be precise, the Schwartz kernel of an operator $A \in \Psi^{m}(\partial M ; \mathcal{S})$ is given by a family of Schwartz kernels $K_{A_{\varepsilon}}=K_{1, \varepsilon}+K_{2, \varepsilon}$ where $K_{1, \varepsilon}$ is conormal of order $m$ at $\Delta_{\text {ad }}$ smoothly down to ff and supported near $\Delta_{\mathrm{ad}}$, and $K_{2, \varepsilon}$ is smooth on the interior and bounded at the boundary hypersurfaces. 
An adiabatic pseudodifferential operator $A \in \Psi^{m}(\partial M ; \mathcal{S})$ with bounds comes with two crucial pieces of data: a principal symbol and a normal operator. The principal symbol $\sigma(A)(\varepsilon)$ is the standard one defined for a conormal distribution, i.e., as a homogeneous section of $N^{*}(\Delta ; \operatorname{End}(\mathcal{S})) \otimes \Omega^{1 / 2}$, the conormal bundle to the lifted diagonal (with coefficients in halfdensities). In our case $N^{*}(\Delta)$ is canonically isomorphic to $T_{\text {ad }}^{*}(\partial M)$, the dual bundle to $T_{\text {ad }}(\partial M)$ defined in (5.2); in particular, the symbol of $A$ is a map $\sigma(A): T_{\mathrm{ad}}^{*}(\partial M) \longrightarrow C^{\infty}(M ; \operatorname{End}(\mathcal{S}))$, well defined only to leading order, and smooth down to ff. The normal operator is the restriction of the Schwartz kernel of $A$ to the front face

$$
N(A)=\left.K_{A}\right|_{\text {ff }}
$$

We thus have maps

$$
\Psi_{\mathrm{ad}}^{m-1}(\partial M ; \mathcal{S}) \hookrightarrow \Psi_{\mathrm{ad}}^{m}(\partial M ; \mathcal{S}) \stackrel{\sigma}{\longrightarrow} S^{m}\left(T_{\mathrm{ad}}^{*}(\partial M ; \mathcal{S})\right) \otimes \Omega^{1 / 2},
$$

and

$$
N: \Psi_{\mathrm{ad}}^{m}(\partial M ; \mathcal{S}) \longrightarrow \Psi_{\mathrm{ff}, \mathrm{ad}}^{m}(\partial M ; \mathcal{S}) .
$$

For fixed $\eta$, we use the same eigenvectors $\phi_{i, \pm}, \partial_{y}^{Z}$ and $i c(\widehat{\eta})$ as in (3.18) above, and consider the spaces

$$
\mathcal{W}_{i}=\operatorname{span}\left\{\phi_{i,+}, \phi_{i,-}\right\}
$$

We have the following.

Lemma 5.4. The layer potential $\mathcal{E}_{\varepsilon}$ is a zero-th order adiabatic family (with bounds), i.e., $\mathcal{E}_{\varepsilon} \in \Psi_{\mathrm{ad}}^{0}(\partial M ; \mathcal{S})$. Using the vectors $\Pi(\eta, i)$ from $(3.25)$, the normal symbol of $\mathcal{E}_{\varepsilon}$ satisfies

$$
\left.\Pi(\eta, i) \widehat{N_{y}\left(\mathcal{E}_{\varepsilon}\right.}\right)(y, \eta) \Pi^{*}(\eta, i)=\mathcal{N}_{\mu_{i},|\eta|},
$$

where

$$
\mathcal{N}_{\mu,|\eta|}=|\eta|\left(\begin{array}{ll}
I_{|\mu+1 / 2|}(|\eta|) K_{|\mu-1 / 2|}(|\eta|) & I_{|\mu+1 / 2|}(|\eta|) K_{|\mu+1 / 2|}(|\eta|) \\
I_{|\mu-1 / 2|}(|\eta|) K_{|\mu-1 / 2|}(|\eta|) & I_{|\mu-1 / 2|}(|\eta|) K_{|\mu+1 / 2|}(|\eta|)
\end{array}\right)
$$

and $I, K$ denote modified Bessel functions.

Proof. That $\mathcal{E}_{\varepsilon}$ is an adiabatic pseudodifferential operator follows from (4.10). The formula in (5.4), (5.5) follows from the Fourier decomposition of the normal operator of the generalized inverse $Q$ in Proposition 3.12, since by (4.3) the operator $\mathcal{E}_{\varepsilon}$ is obtained by taking the limit in $(3.26)$ as $\sigma=x / x^{\prime} \uparrow 1$ and checking that $\lim _{\sigma \uparrow 1} \mathcal{M}_{\mu,|\eta|}(\sigma, 1)=\mathcal{N}_{\mu,|\eta|}$.

\subsection{APS projections as an adiabatic family}

To study the integral kernel of the projector $\pi_{\mathrm{APS}, \varepsilon}$ we will make use of the fact that the boundary

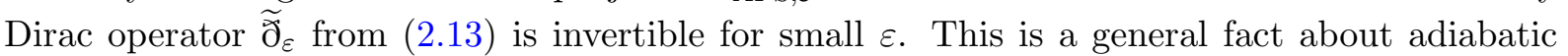
pseudodifferential operators: invertibility at $\varepsilon=0$ implies invertibility for small epsilon, or formally

Theorem 5.5. Let $A_{\varepsilon} \in \Psi_{\mathrm{ad}}^{m}(M ; \mathcal{S})$ and assume that on each fiber $\mathrm{ff}_{y}$ the Fourier transform of the normal operator $\widehat{N\left(A_{\varepsilon}\right)}$ y is invertible on $L^{2}\left(Z ; \mathcal{S}_{y}, k_{y}\right)$, with $\mathcal{S}_{y}$ the restriction of the spinor bundle to the fiber over $y$ and $k_{y}=\left.g_{N / Y}\right|_{y}$. Then $A_{\varepsilon}$ is invertible for small $\varepsilon$. 
It is well known [8] that for each fixed $\varepsilon>0, \pi_{\mathrm{APS}, \varepsilon}$ is a pseudodifferential operator of order 0 . As $\varepsilon$ varies, these operators form an adiabatic family:

Lemma 5.6. The family $\pi_{\mathrm{APS}, \varepsilon}$ lies in $\Psi_{\mathrm{ad}}^{0}(\partial M ; \mathcal{S})$. Its normal symbol $N\left(\pi_{\mathrm{APS}, \varepsilon}\right)$ satisfies

$$
N\left(\pi_{\mathrm{APS}, \varepsilon}\right)=\frac{1}{2} N\left(\widetilde{\widetilde{\mathrm{d}}}_{\varepsilon}\right)^{-1}\left(N\left(\widetilde{\widetilde{\mathrm{d}}}_{\varepsilon}\right)-\left|N\left(\widetilde{\widetilde{\mathrm{d}}}_{\varepsilon}\right)\right|\right) .
$$

Proof. By Assumption 3.1, $\widetilde{\mathrm{d}}_{\varepsilon}$ is invertible for small $\varepsilon$. Indeed, by (5.8), $N\left(\widetilde{\widetilde{\partial}}_{\varepsilon}\right)_{y}$ does not have zero as an eigenvalue. The projectors $\pi_{\mathrm{APS}, \varepsilon}$ can be expressed in terms of functions of the tangential operators $\widetilde{\widetilde{\partial}}_{\varepsilon}[8]$ via the formula

$$
\pi_{\mathrm{APS}, \varepsilon}=\frac{1}{2} \widetilde{\mathrm{\partial}}_{\varepsilon}^{-1}\left(\widetilde{\widetilde{\mathrm{d}}}_{\varepsilon}-\left|\widetilde{\widetilde{\mathrm{d}}}_{\varepsilon}\right|\right) .
$$

Following [59], the operator $\widetilde{\mathrm{d}}_{\varepsilon}^{-1}\left|\widetilde{\mathrm{d}}_{\varepsilon}\right|$ is in $\Psi_{\text {ad }}^{1}(\partial X ; \mathcal{S})$ and has the expected normal operator, namely the one obtained by applying the appropriate functions to the normal operator of $N\left(\widetilde{\widetilde{\partial}}_{\varepsilon}\right)_{y}$ and composing them.

We compute that the operator $\widehat{N\left(\widetilde{\mathrm{d}}_{\varepsilon}\right)_{y}}$ acts on the spaces $\mathcal{W}_{i}$ from $(5.3)$ by

$$
\widehat{N\left(\widetilde{\jmath}_{\varepsilon}\right)_{y}}(\eta) \phi_{i, \pm}= \pm \mu \phi_{i, \pm}-|\eta| \phi_{i, \mp} \text {. }
$$

That is to say, with $\Pi(\eta, i)$ as in (3.25),

$$
\Pi(\eta, i) \widehat{N\left(\widetilde{\widetilde{d}}_{\varepsilon}\right)_{y}}(\eta) \Pi^{*}(\eta, i)=\left(\begin{array}{cc}
\mu & -|\eta| \\
-|\eta| & -\mu
\end{array}\right) .
$$

Thus,

$$
\Pi(\eta, i) N\left(\widehat{\widetilde{\widetilde{\partial}}_{\varepsilon}^{-1} \mid \widetilde{\widetilde{\partial}}_{\varepsilon}} \mid\right)_{y}(\eta) \Pi^{*}(\eta, i)=\frac{1}{\left(\mu^{2}+|\eta|^{2}\right)^{1 / 2}}\left(\begin{array}{cc}
\mu & -|\eta| \\
-|\eta| & -\mu
\end{array}\right) \Pi_{\mu_{i}, j} .
$$

Using (5.7), we obtain

$$
\Pi(\eta, i) \widehat{N}\left(\pi_{\mathrm{APS}, \varepsilon}\right)_{y}(\eta) \Pi^{*}(\eta, i)=\mathcal{N}_{\mu,|\eta|}^{\mathrm{APS}},
$$

where

$$
\mathcal{N}_{\mu,|\eta|}^{\mathrm{APS}}=\frac{1}{2}\left(\operatorname{Id}_{2 \times 2}+\frac{1}{\left(\mu^{2}+|\eta|^{2}\right)^{1 / 2}}\left(\begin{array}{cc}
-\mu & |\eta| \\
|\eta| & \mu
\end{array}\right)\right) .
$$

Theorem 5.7. There exists a smooth family $\pi_{\varepsilon, t}$, parametrized by $t \in[0,1]$ satisfying:

1) for fixed $t, \pi_{\varepsilon, t} \in \mathrm{Gr}_{\mathrm{APS}, \varepsilon}$, the Grassmanians defined in (5.1), and

2) $\pi_{\varepsilon, 0}=\mathcal{E}_{\varepsilon}$ and $\pi_{\varepsilon, 1}=\pi_{\mathrm{APS}, \varepsilon}$.

Proof. The proof proceeds in two main steps. First, we construct a homotopy from the normal operators $N\left(\mathcal{E}_{\varepsilon}\right)$ to $N\left(\pi_{\mathrm{APS}, \varepsilon}\right)$. Then we extend this homotopy to a homotopy of the adiabatic families as claimed in the theorem.

For the homotopy of the normal operators, the main lemma will be the following

Claim 5.8. For each $y \in Y$, the normal operators $N\left(\mathcal{E}_{\varepsilon}\right)_{y}$ and $N\left(\pi_{\mathrm{APS}, \varepsilon}\right)$, acting on $L^{2}(Z \times$ $\left.T_{y} Y ; \mathcal{S}_{y}\right)$, satisfy

$$
\left\|N\left(\mathcal{E}_{\varepsilon}\right)_{y}-N\left(\pi_{\mathrm{APS}, \varepsilon}\right)\right\|_{L^{2} \longrightarrow L^{2}}<1-\delta,
$$

for some $\delta>0$ independent of $y$. 
Assuming the claim for the moment, the following argument from [17, Chapter 15] furnishes a homotopy. In general, let $P$ and $Q$ be projections on a separable Hilbert space. Define $T_{t}=\mathrm{Id}+t(Q-P)(2 P-\mathrm{Id})$, and note that $T_{1} P=Q T_{1}$. Now assume that $T_{t}$ is invertible for all $t$. Then the operator

$$
F_{t}=T_{t}^{-1} P T_{t}
$$

is a homotopy from $P$ to $Q$, i.e., $F_{0}=P, F_{1}=Q$. This holds in particular if $\|P-Q\|<1$, in which case $T_{t}$ is invertible by Neumann series for $t \in[0,1]$.

To apply this in our context, we first take $P=N\left(\mathcal{E}_{\varepsilon}\right)_{y}$ and $Q=N\left(\pi_{\mathrm{APS}, \varepsilon}\right)_{y}$, and see that the corresponding operator $T_{t}$ is invertible by Claim 5.8. Now taking $P=\mathcal{E}_{\varepsilon}$ and $Q=\pi_{\mathrm{APS}, \varepsilon}$ (so $P, Q$, and $T_{t}$ depend on $\varepsilon$ ) by Theorem $5.5, T_{t}$ is invertible for small $\varepsilon$. Thus the homotopy $F_{t}=F_{t}(\varepsilon)=: \pi_{\varepsilon, t}$ is well defined for small $\varepsilon$. In fact, $\pi_{\varepsilon, t}$ is a smooth family of adiabatic pseudodifferential projections with principal symbol equal to that of $\pi_{\mathrm{APS}, \varepsilon}$ for all $\varepsilon$.

Thus it remains to prove Claim 5.8. By the formulas for the normal operators given in (5.4) and (5.6) and Plancherel, the claim will follow if we can show that for each $\mu$ with $|\mu|>1 / 2$, and all $|\eta|$, that

$$
\left\|\mathcal{N}_{\mu,|\eta|}-\mathcal{N}_{\mu,|\eta|}^{\mathrm{APS}}\right\|<1-\delta
$$

for some $\delta$ independent of $\mu \geq 1 / 2$ and $|\eta|$. Here the norm is as a map of $\mathbb{R}^{2}$ with the standard Euclidean norm. We prove the bound in (5.9) using standard bounds on modified Bessel functions in Appendix A.

\section{Proof of Main Theorem: limit of the index formula}

Recall (e.g., [51, Section 2.14]) that if $E \longrightarrow M$ is a real vector bundle of rank $k$, with connection $\nabla^{E}$ and curvature tensor $R^{E}$ then every smooth function (or formal power series)

$$
P: \mathfrak{s o}(k) \longrightarrow \mathbb{C},
$$

that is invariant under the adjoint action of $S O(k)$, determines a closed differential form $P\left(R^{E}\right) \in$ $\mathcal{C}^{\infty}\left(M ; \Lambda^{*} T^{*} M\right)$. If $\nabla_{1}^{E}$ is another connection on $E$, with curvature tensor $R_{1}^{E}$ then $P\left(R^{E}\right)$ and $P\left(R_{1}^{E}\right)$ differ by an exact form. Indeed, define a family of connections on $E$ by

$$
\theta=\nabla_{1}^{E}-\nabla^{E} \in \mathcal{C}^{\infty}\left(M ; T^{*} M \otimes \operatorname{Hom}(E)\right), \quad \nabla_{t}^{E}=(1-t) \nabla^{E}+t \nabla_{1}^{E}=\nabla^{E}+t \theta,
$$

denote the curvature of $\nabla_{t}^{E}$ by $R_{t}^{E}$, and let

$$
P^{\prime}(A ; B)=\left.\frac{\partial}{\partial s}\right|_{s=0} P(A+s B) .
$$

The differential form

$$
T P\left(\nabla^{E}, \nabla_{1}^{E}\right)=\int_{0}^{1} P^{\prime}\left(R_{t}^{E} ; \theta\right) d t
$$

satisfies

$$
d T P\left(\nabla^{E}, \nabla_{1}^{E}\right)=P\left(R^{E}\right)-P\left(R_{1}^{E}\right) .
$$

Now consider for $\varepsilon<1$ the truncated manifold $M_{\varepsilon}=\{x \geq \varepsilon\}$ and the corresponding truncated collar neighborhood $\mathscr{C}_{\varepsilon}=[\varepsilon, 1] \times N$. Let $\nabla^{\mathrm{pt}}$ be the Levi-Civita connection of the metric

$$
g_{\mathrm{pt}}=d x^{2}+\varepsilon^{2} g_{Z}+\phi^{*} g_{Y}
$$


The Atiyah-Patodi-Singer index theorem on $M_{\varepsilon}$ has the form [34, 35], cf. [28]

$$
\int_{M_{\varepsilon}} A S(\nabla)+\int_{\partial M_{\varepsilon}} T A S\left(\nabla, \nabla^{\mathrm{pt}}\right)-\frac{1}{2} \eta\left(\partial M_{\varepsilon}\right)
$$

where $A S$ is a characteristic form associated to a connection $\nabla$ and $T A S\left(\nabla, \nabla^{\mathrm{pt}}\right)$ is its transgression form with respect to the connection $\nabla^{\mathrm{pt}}$.

The Levi-Civita connection of $g_{\mathrm{pt}}$ induces a connection on $T_{\mathrm{ie}} M_{\varepsilon}$, which we continue to denote $\nabla^{\mathrm{pt}}$. Let $\theta^{\varepsilon}=\nabla-\nabla^{\mathrm{pt}}$. Since $g_{\mathrm{ie}}$ and $g_{\mathrm{pt}}$ coincide on $\{x=\varepsilon\}$ we have

$$
\left.g_{\mathrm{pt}}\left(\nabla_{A}^{\mathrm{pt}} B, C\right)\right|_{x=\varepsilon}=\left.g_{\mathrm{ie}}\left(\nabla_{A}^{\mathrm{ie}} B, C\right)\right|_{x=\varepsilon} \quad \text { if } \quad A, B, C \in \mathcal{C}^{\infty}\left(\mathscr{C}_{\varepsilon} ; T N\right) .
$$

On the other hand, if $A, B, C \in\left\{\partial_{x}, \frac{1}{x} V, \widetilde{U}\right\}$, we have

$$
g_{\mathrm{pt}}\left(\nabla_{A}^{\mathrm{pt}} B, C\right)=0 \quad \text { if } \quad \partial_{x} \in\{A, B, C\}
$$

except for

$$
g_{\mathrm{pt}}\left(\nabla_{\partial_{x}}^{\mathrm{pt}} \frac{1}{x} V_{1}, \frac{1}{x} V_{2}\right)=-\frac{\varepsilon^{2}}{x^{3}} g_{Z}\left(V_{1}, V_{2}\right)
$$

Note that, analogously to (2.7), we have

$$
j_{0}^{*} \nabla^{\mathrm{pt}}=j_{0}^{*} \nabla^{v} \oplus j_{0}^{*} \nabla^{h},
$$

where, as above, $j_{\varepsilon}: N \hookrightarrow \mathscr{C}$ is the inclusion of $\{x=\varepsilon\}$, and $\nabla^{v}=\mathbf{v} \circ \nabla \circ \mathbf{v}$ is the restriction of the Levi-Civita connection to $T N / Y$.

Thus

$$
\left.\theta_{A}^{\varepsilon}(B)\right|_{x=\varepsilon}=0
$$

except for

$$
\left.\theta_{\partial_{x}}^{\varepsilon}\left(\frac{1}{x} V\right)\right|_{x=\varepsilon}=\frac{1}{\varepsilon} \frac{1}{x} V,\left.\quad \theta_{V}^{\varepsilon}\left(\partial_{x}\right)\right|_{x=\varepsilon}=\frac{1}{x} V,\left.\quad \theta_{V_{1}}^{\varepsilon}\left(\frac{1}{x} V_{2}\right)\right|_{x=\varepsilon}=-g_{Z}\left(V_{1}, V_{2}\right) \partial_{x} .
$$

In particular note that $j_{\varepsilon}^{*} \theta^{\varepsilon}$ is independent of $\varepsilon$ and is equal to

$$
j_{\varepsilon}^{*} \theta^{\varepsilon}=j_{0}^{*} \nabla^{v_{+}}-j_{0}^{*} \nabla^{v} .
$$

Next we need to compute the restriction to $x=\varepsilon$ of the curvature $\Omega_{t}$ of the connection $(1-t) \nabla+t \nabla^{\mathrm{pt}}=\nabla+t \theta^{\varepsilon}$. Locally, with $\omega$ the local connection one-form of $\nabla$ (2.8), the curvature $\Omega_{t}$ is given by

$$
\Omega_{t}=d\left(\omega+t \theta^{\varepsilon}\right)+\left(\omega+t \theta^{\varepsilon}\right) \wedge\left(\omega+t \theta^{\varepsilon}\right)=\Omega+t\left(d \theta^{\varepsilon}+\left[\omega, \theta^{\varepsilon}\right]_{s}\right)+t^{2} \theta^{\varepsilon} \wedge \theta^{\varepsilon},
$$

where $[\cdot, \cdot]_{s}$ denotes the supercommutator with respect to form parity, so that $\left[\omega, \theta^{\varepsilon}\right]_{s}=\omega \wedge \theta^{\varepsilon}+$ $\theta^{\varepsilon} \wedge \omega$. In terms of the splitting (2.6) we have

$$
\left.\Omega\right|_{x=\varepsilon}=\left(\begin{array}{cc}
\Omega_{v_{+}} & \mathcal{O}(\varepsilon) \\
\mathcal{O}(\varepsilon) & \phi^{*} \Omega_{Y}
\end{array}\right), \quad \omega=\left(\begin{array}{cc}
\omega_{v_{+}} & \mathcal{O}(x) \\
\mathcal{O}(x) & \phi^{*} \omega_{Y}+\mathcal{O}\left(x^{2}\right)
\end{array}\right), \quad j_{\varepsilon}^{*} \theta^{\varepsilon}=\left(\begin{array}{cc}
\widetilde{\theta} & 0 \\
0 & 0
\end{array}\right)
$$

and hence

$$
\left.\Omega_{t}\right|_{x=\varepsilon}=\left(\begin{array}{cc}
\Omega_{v_{+}}+t\left(d \tilde{\theta}+\left[\omega_{v_{+}}, \widetilde{\theta}\right]_{s}\right)+t^{2} \widetilde{\theta} \wedge \widetilde{\theta} & \mathcal{O}(\varepsilon) \\
\mathcal{O}(\varepsilon) & \phi^{*} \Omega_{Y}
\end{array}\right) .
$$


In particular, if we denote $\Omega_{v_{+}, t}$ the curvature of the connection $(1-t) \nabla^{v_{+}}+t \nabla^{v}$ on the bundle $\left\langle\partial_{x}\right\rangle+T N / Y$, we have

$$
j_{\varepsilon}^{*} \Omega_{t}=j_{0}^{*} \Omega_{t}+\mathcal{O}(\varepsilon), \quad \text { with } \quad j_{0}^{*} \Omega_{t}=\left(\begin{array}{cc}
\Omega_{v_{+}, t} & 0 \\
0 & \phi^{*} \Omega_{Y}
\end{array}\right) .
$$

It follows that

$$
\lim _{\varepsilon \rightarrow 0} j_{\varepsilon}^{*} T \widehat{A}\left(\nabla, \nabla^{\mathrm{pt}}\right)=\left.\int_{0}^{1} \frac{\partial}{\partial s}\right|_{s=0} j_{0}^{*} \widehat{A}\left(\Omega_{Y}\right) \widehat{A}\left(\Omega_{v_{+}, t}+s \widetilde{\theta}\right) d t=\widehat{A}(Y) \wedge T \widehat{A}\left(\nabla^{v_{+}}, \nabla^{v}\right)
$$

and similarly for any multiplicative characteristic class.

We can now prove the main theorem, whose statement we recall for the reader's convenience.

Theorem 6.1. Let $X$ be stratified space with a single singular stratum endowed with an incomplete edge metric $g$ and let $M$ be its resolution. If $\partial$ is a Dirac operator associated to a spin bundle $\mathcal{S} \longrightarrow M$ and $\mathrm{\partial}$ satisfies Assumption 3.1, then

$$
\text { Ind }\left(\text { ə : } \mathcal{D}^{+} \longrightarrow L^{2}\left(M ; \mathcal{S}^{-}\right)\right)=\int_{M} \widehat{A}(M)+\int_{Y} \widehat{A}(Y)\left(-\frac{1}{2} \widehat{\eta}\left(\varlimsup_{Z}\right)+\int_{\partial M / Y} T \widehat{A}\left(\nabla^{v_{+}}, \nabla^{\mathrm{pt}}\right)\right) \text {, }
$$

where $\widehat{A}$ denotes the $\widehat{A}$-genus, $T \widehat{A}\left(\nabla^{v_{+}}, \nabla^{\mathrm{pt}}\right)$ denotes the transgression form of the $\widehat{A}$ genus associated to the connections $\nabla^{v_{+}}$and $\nabla^{\mathrm{pt}}$ above, and $\widehat{\eta}$ the $\eta$-form of Bismut-Cheeger [12].

Proof of Main Theorem. Combining Theorems 4.1 and 5.1 we know that, for $\varepsilon$ small enough,

$$
\begin{aligned}
\text { Ind }\left(ð: \mathcal{D}^{+} \longrightarrow L^{2}\left(M ; \mathcal{S}^{-}\right)\right) & =\operatorname{Ind}\left(ð: \mathcal{D}_{\varepsilon}^{+} \longrightarrow L^{2}\left(M_{\varepsilon} ; \mathcal{S}^{-}\right)\right) \\
& =\operatorname{Ind}\left(ð: \mathcal{D}_{\mathrm{APS}, \varepsilon}^{+} \longrightarrow L^{2}\left(M_{\varepsilon} ; \mathcal{S}^{-}\right)\right) .
\end{aligned}
$$

Hence

$$
\begin{aligned}
\operatorname{Ind}\left(ð: \mathcal{D}^{+} \longrightarrow L^{2}\left(M ; \mathcal{S}^{-}\right)\right) & =\lim _{\varepsilon \rightarrow 0} \operatorname{Ind}\left(ð: \mathcal{D}_{\mathrm{APS}, \varepsilon}^{+} \longrightarrow L^{2}\left(M_{\varepsilon} ; \mathcal{S}^{-}\right)\right) \\
& =\lim _{\varepsilon \rightarrow 0} \int_{M_{\varepsilon}} \widehat{A}(\nabla)+\int_{\partial M_{\varepsilon}} T \widehat{A}\left(\nabla, \nabla^{\mathrm{pt}}\right)-\frac{1}{2} \eta\left(\partial M_{\varepsilon}\right) \\
& =\int_{M} \widehat{A}(M)+\int_{\partial M} \widehat{A}(Y) \wedge T \widehat{A}\left(\nabla^{v_{+}}, \nabla^{v}\right)-\frac{1}{2} \int_{Y} \widehat{A}(Y) \widehat{\eta}\left(\varlimsup_{Z}\right) .
\end{aligned}
$$

\subsection{Four-dimensions with circle fibers}

An incomplete edge space whose link is a sphere is topologically a smooth space. So let us consider a four-dimensional manifold $X$ with a submanifold $Y$ and a Riemannian metric on $X \backslash Y$ that in a tubular neighborhood of $Y$ takes the form

$$
d x^{2}+x^{2} \beta^{2} d \theta^{2}+\phi^{*} g_{Y}
$$

Here $\beta$ is a constant and $2 \pi \beta$ is the 'cone angle' along the edge.

Recall that the circle has two distinct spin structures, and with the round metric the corresponding Dirac operators have spectra equal to either the even or odd integer multiples of $\pi$. The non-trivial spin structure on the circle is the one that extends to the disk, and so any spin structure on $X$ will induce non-trivial spin structures on its link circles. Thus, cf. [26, Proposition 2.1], the generalized Witt assumption will be satisfied as long as $\beta \leq 1$. 
In this setting the relevant characteristic class is the first Pontryagin class: for a two-by-two anti-symmetric matrix $A$, let

$$
p_{1}(A)=-c_{2}(A)=-\frac{1}{8 \pi^{2}} \operatorname{Tr}\left(A^{2}\right) .
$$

Note that $p_{1}^{\prime}(A ; B)=-\frac{1}{(2 \pi)^{2}} \operatorname{Tr}(A B)$, and so

$$
T p_{1}\left(\nabla, \nabla^{\mathrm{pt}}\right)=-\frac{1}{(2 \pi)^{2}} \int_{0}^{1} \operatorname{Tr} j_{0}^{*}\left(\theta \wedge \Omega_{t}\right) d t
$$

with $\Omega_{t}=\Omega+t\left(d \theta^{\varepsilon}+\left[\omega, \theta^{\varepsilon}\right]_{s}\right)+t^{2} \theta^{\varepsilon} \wedge \theta^{\varepsilon}$. We can simplify this formula. Indeed, note that if $\left\{V_{i}\right\}$ are an orthonormal frame for $T N / Y$ then

$$
j_{\varepsilon}^{*}\left(\theta^{\varepsilon} \wedge \theta^{\varepsilon}\right)=\sum \Theta_{i j} V_{i}^{b} \wedge V_{j}^{b} \quad \text { with } \quad \Theta_{i j}\left(\frac{1}{x} V_{k}\right)=-\delta_{k j} \frac{1}{x} V_{i},
$$

and so in particular $\operatorname{dim} Z=1$ implies $j_{\varepsilon}^{*}\left(\theta^{\varepsilon} \wedge \theta^{\varepsilon}\right)=0$. Moreover with respect to the splitting (2.5), $\theta$ is off-diagonal and $\Omega$ is on-diagonal, hence $\operatorname{Tr} j_{0}^{*}(\theta \wedge \Omega)=0$ and

$$
T p_{1}\left(\nabla, \nabla^{\mathrm{pt}}\right)=-\frac{1}{4 \pi^{2}} \int_{0}^{1} t \operatorname{Tr} j_{0}^{*}(\theta \wedge d \theta) d t=-\frac{1}{8 \pi^{2}} \operatorname{Tr} j_{0}^{*}(\theta \wedge d \theta) .
$$

Next let us consider $\theta$ in more detail. From (6.1), with respect to the splitting (2.5), we have

$$
j_{0}^{*} \theta=\left(\begin{array}{ccc}
0 & \mathrm{Id} & 0 \\
-\mathrm{Id} & 0 & 0 \\
0 & 0 & 0
\end{array}\right) \alpha
$$

where $\alpha$ is a vertical one-form of $g_{Z}$ length one. This form is closely related to the 'global angular form' described in [18, p. 70]. Indeed, $\alpha$ restricts to each fiber to be $\beta d \theta$ which integrates out to $2 \pi \beta$. It follows that $d \alpha=-2 \pi \beta \phi^{*} e$, where $e \in \mathcal{C}^{\infty}\left(Y ; T^{*} Y\right)$ is the Euler class of $Y$ as a submanifold of $X$, and hence

$$
j_{0}^{*}(\theta \wedge d \theta)=\left(\begin{array}{ccc}
-\mathrm{Id} & 0 & 0 \\
0 & -\mathrm{Id} & 0 \\
0 & 0 & 0
\end{array}\right) \alpha \wedge\left(-2 \pi \beta \phi^{*} e\right) .
$$

Thus we find

$$
\begin{aligned}
\int_{\partial M} T p_{1}\left(\nabla, \nabla^{\mathrm{pt}}\right) & =-\frac{1}{8 \pi^{2}} \int_{\partial M} \operatorname{Tr} j_{0}^{*}(\theta \wedge d \theta) \\
& =-\frac{1}{8 \pi^{2}} \int_{\partial M}\left(4 \pi \beta \alpha \wedge \phi^{*} e\right)=-\beta^{2} \int_{Y} e=-\beta^{2}[Y]^{2} .
\end{aligned}
$$

This computation yields a formula for the index of the Dirac operator and, combined with results of Dai and Dai-Zhang, also a proof of the signature theorem of Atiyah-LeBrun.

Theorem 6.2. Let $X$ be an oriented four-dimensional manifold, $Y$ a smooth compact oriented embedded surface, and $g$ an incomplete edge metric on $X \backslash Y$ with cone angle $2 \pi \beta$ along $Y$.

1) If $X$ is spin and $\beta \in(0,1]$,

$$
\text { Ind }\left(\text { ð : } \mathcal{D}^{+} \longrightarrow L^{2}\left(M ; \mathcal{S}^{-}\right)\right)=-\frac{1}{24} \int_{M} p_{1}(M)+\frac{1}{24}\left(\beta^{2}-1\right)[Y]^{2} \text {. }
$$


2) The signature of $X$ is given by (see Atiyah-LeBrun [7])

$$
\operatorname{sgn}(X)=\frac{1}{12 \pi^{2}} \int_{M}\left(\left|W_{+}\right|^{2}-\left|W_{-}\right|^{2}\right) d \mu+\frac{1-\beta^{2}}{3}[Y]^{2}
$$

Proof. 1) As mentioned above, the fact that the spin structure extends to all of $X$ and $\beta \in(0,1]$ implies that the generalized Witt assumption for $\partial$ is satisfied. The degree four term of the $\hat{A}$ genus is $-p_{1} / 24$, so applying our index formula (1.4) and using the derivation of the local boundary term for $p_{1}$ in $(6.2)$ gives

$$
\text { Ind }\left(ð: \mathcal{D}^{+} \longrightarrow L^{2}\left(M ; \mathcal{S}^{-}\right)\right)=-\frac{1}{24} \int_{M} p_{1}(M)-\frac{1}{24} \beta^{2}[Y]^{2}+\int_{Y} \widehat{A}(Y)\left(-\frac{1}{2} \widehat{\eta}\left(\partial_{Z}\right)\right),
$$

where the final term on the right is the limit $(1 / 2) \lim _{\varepsilon \rightarrow 0} \eta_{\varepsilon}$ where $\varepsilon_{\varepsilon}$ is the eta-invariants induced on the boundary of $M_{\varepsilon}$ as $\varepsilon \rightarrow 0$. Thus we claim (and it remains to prove) that the adiabatic limit of the eta-invariant for the spin Dirac operator is

$$
\lim _{\varepsilon \rightarrow 0} \frac{1}{2} \eta_{\varepsilon}=\frac{1}{24}[Y]^{2},
$$

i.e., the limit of the eta-invariants is the opposite of the local boundary term when $\beta=1$, which indeed it should be since in that case the metric is smooth across $x=0$.

Although other derivations of the adiabatic eta invariant exist [29], we prefer to give on here which we find intuitive and which fits nicely with arguments above. To this end, we consider $N$, a disc bundle over a smooth manifold $Y$, and we assume $N$ is spin. We will show below that $N$ admits a positive scalar curvature metric. Thus, given a spin structure and metric, the index of $\partial$ vanishes on $N$. If we furthermore note that $N$ is diffeomorphic to $[0,1)_{x} \times X$ where $X$ is a circle bundle over $Y$, and let $N^{\varepsilon}=[0, \varepsilon)_{x} \times X$, we may consider metrics

$$
g=d x^{2}+f^{2}(x) k+h,
$$

where $h$ is the pullback of a metric on $Y, k \in \operatorname{Sym}^{0,2}\left(N^{\varepsilon}\right) x$ and $d x$-independent and restricts to a Riemannian metric on the fibers of $X$. We assume $f$ is smooth across $x=0$ with $f(x)=$ $x+O\left(x^{2}\right)$ which implies that $g$ is smooth on $N$. Using the computation of the connection above, with respect to the orthonormal basis, $X_{i}, \frac{1}{f} U, \partial_{x}$ the connection one form of $g$ is

$$
\omega=\left(\begin{array}{c|c|c}
\widetilde{\omega}_{\widetilde{h}}-f^{2} \frac{1}{2} g^{\partial} \mathcal{R} & -f g^{\partial}\left(\widehat{I I}+\frac{1}{2} \widehat{\mathcal{R}}\right) & 0 \\
\hline f g^{\partial}\left(\widehat{I I}+\frac{1}{2} \widehat{\mathcal{R}}\right) & 0 & f^{\prime} U^{\sharp} \\
\hline 0 & -f^{\prime} U^{\sharp} & 0
\end{array}\right),
$$

where $g^{\partial}=k+h$ is the metric on the circle bundle $X$. We will take

$$
f(x)=f_{\varepsilon}(x)=x \chi_{\varepsilon}(x),
$$

where $\chi$ is a smooth positive function that is monotone decreasing with $\chi(x)=1$ for $x \leq 1 / 3$ and $\chi(x)=\beta$ for $x \geq 2 / 3$. Then $f=f^{\prime}=f^{\prime \prime}=O(1 / \varepsilon)$, and using $\Omega=d \omega+\omega \wedge \omega$, we see that

$$
\widehat{A}_{g}=F d \operatorname{Vol}_{g}
$$

where $F$ is a function that is $O(1 / \varepsilon)$. Since $\operatorname{Vol}\left(N_{\varepsilon}\right)=O\left(\varepsilon^{2}\right)$,

$$
\int_{N_{\varepsilon}} \widehat{A}_{\varepsilon}=-\frac{1}{24} \int_{N_{\varepsilon}} p_{1} \rightarrow 0 \quad \text { as } \quad \varepsilon \rightarrow 0 .
$$


Since the index of the Dirac operator vanishes on $N^{\varepsilon}$, applying the APS formula gives

$$
0=-\frac{1}{24} \int_{N^{\varepsilon}} p_{1}+\int_{\partial N^{\varepsilon}} T p_{1}\left(\nabla, \nabla^{\mathrm{pt}}\right)-\frac{1}{2} \eta\left(\partial N^{\varepsilon}\right),
$$

where $\nabla^{\mathrm{pt}}$ is as in (1.3), and thus the limit of the trangression forms is exactly as computed above. Thus by taking the $\varepsilon \rightarrow 0$ limit we obtain (6.3).

To prove part 1) it remains to prove the existence of a positive scalar curvature metric on $N$. To this end we take the metric $g$ as in (6.4) on $N^{\varepsilon}$ now with

$$
f(x)=f_{\delta}(x)=\delta \sin (x / \delta) .
$$

Note that $f=O(\varepsilon), f^{\prime}=O(\varepsilon / \delta)$. Then curvature equals

$$
\Omega=d \omega+\omega \wedge \omega=\left(\begin{array}{c|c|c}
\widetilde{\Omega}_{\widetilde{h}} & 0 & 0 \\
\hline 0 & 0 & f^{\prime \prime} d x \wedge U^{\sharp} \\
\hline 0 & -f^{\prime \prime} d x \wedge U^{\sharp} & 0
\end{array}\right)+O(\varepsilon)+O(\varepsilon / \delta) .
$$

Denoting our orthonormal basis by $e_{i}, i=1, \ldots, n$ and taking traces gives

$$
\operatorname{scal}_{g}=\delta^{i k} \delta^{j l} \Omega_{i j}\left(e_{k}, e_{l}\right)=\operatorname{scal}_{h}+\frac{2}{\delta^{2}}+O(\varepsilon / \delta),
$$

and thus taking $\varepsilon / \delta=1$ and $\delta$ small gives a positive scalar curvature metric.

2) Since $X$ is a smooth manifold we can use Novikov additivity of the signature to decompose the signature as

$$
\operatorname{sgn}(X)=\operatorname{sgn}\left(X \backslash M_{\varepsilon}\right)+\operatorname{sgn}\left(M_{\varepsilon}\right) .
$$

Identifying $X \backslash M_{\varepsilon}$ with a disk bundle over $Y$ we have from [27, p. 314] that

$$
\operatorname{sgn}\left(X \backslash M_{\varepsilon}\right)=\operatorname{sgn}\left(\int_{Y} e\right)
$$

i.e., the signature is the sign of the self-intersection number of $Y$ in $X$. In fact this is a simple exercise using the Thom isomorphism theorem.

The Atiyah-Patodi-Singer index theorem for the signature of $M_{\varepsilon}$ yields

$$
\operatorname{sgn}\left(M_{\varepsilon}\right)=\frac{1}{3} \int_{M_{\varepsilon}} p_{1}(\nabla)+\frac{1}{3} \int_{\partial M_{\varepsilon}} T p_{1}\left(\nabla, \nabla^{\mathrm{pt}}\right)-\eta_{\varepsilon}^{\mathrm{even}},
$$

where $\eta_{\varepsilon}^{\text {even }}$ is the eta-invariant of the boundary signature operator restricted to forms of even degree. As $\varepsilon \rightarrow 0$, the eta invariant is undergoing adiabatic degeneration and its limit is computed in [29, Theorem 3.2],

$$
\lim _{\varepsilon \rightarrow 0}=-\int_{Y} L(T Y)\left(\operatorname{coth} e-e^{-1}\right)+\operatorname{sgn}\left(B_{e}\right),
$$

where $B_{e}$ is the bilinear form on $H^{0}(Y)$ given by $H^{0}(Y) \ni c, c^{\prime} \mapsto c c^{\prime}\langle e, Y\rangle \in \mathbb{R}$, i.e., it is again the sign of the self-intersection of $Y$. (In comparing with [29] note that the orientation of $\partial M_{\varepsilon}$ is the opposite of the orientation of the spherical normal bundle of $Y$ in $X$, and so $\operatorname{sgn}\left(B_{e}\right)=-\operatorname{sgn}\left(X \backslash M_{\varepsilon}\right)$.) The only term in $L(T Y)\left(\operatorname{coth} e-e^{-1}\right)$ of degree two is $\frac{1}{3} e$, and hence

$$
\operatorname{sgn}(X)=\frac{1}{3} \int_{X} p_{1}+\frac{1}{3}[Y]^{2}+\frac{1}{3}\left(-\beta^{2}[Y]^{2}\right)
$$

as required. (Note that we could also argue as in the Dirac case to compute the limit of the eta invariants.) 


\section{Positive scalar curvature metrics}

In this short section, we prove Theorem 1.3 following [25]. We recall the statement of the theorem for the convenience of the reader:

Theorem. Let $(M, g)$ be a spin space with an incomplete edge metric. The 'geometric Witt assumption' (1.2) holds if either:

1. $\operatorname{dim} Z \geq 2$ and the scalar curvature of $g$ is non-negative in a neighborhood of $\partial M$.

2. $\operatorname{dim} Z=1$, the spin structure on $M$ is the lift of a spin structure on $X$, and the cone angle satisfies $2 \pi \beta \leq 2 \pi$.

If the geometric Witt assumption holds and in addition the scalar curvature of $g$ is nonnegative on all of $M$, and positive somewhere, then $\operatorname{Ind}(\widetilde{\partial})=0$.

Proof. 1) Taking traces in (2.9), the scalar curvature $R_{g}$ satisfies

$$
R_{g}=R_{\text {cone }}+\mathcal{O}(1)
$$

where $R_{\text {cone }}$ is the scalar curvature of the cone with metric $d x^{2}+\left.x^{2} g_{N / Y}\right|_{\left\langle\partial_{x}\right\rangle \oplus \frac{1}{x} T N / Y}$, as in (2.6). On the other hand, by [25, Section 4], the scalar curvature of an exact cone $C(Z)$ is equal to $x^{-2}\left(R_{Z}-\operatorname{dim}(Z)(\operatorname{dim}(Z)-1)\right)$, where $R_{Z}$ is the scalar curvature of $Z$. Thus $R_{g} \geq 0$ implies that $R_{Z} \geq \operatorname{dim}(Z)(\operatorname{dim}(Z)-1)$, which by [25, Lemma 3.5] shows that Assumption 3.1 holds.

2) In the circle fiber case, there exist local trivializations of the boundary fibration such that $\mathrm{\partial}_{y}^{Z}=\frac{1}{\beta^{2}} \partial_{\theta}$ where $\partial_{\theta}$ is the Dirac operator on $\mathbb{S}^{1}$ for the spin structure that bounds a disk. Since $\operatorname{spec}\left(\varpi_{\theta}\right) \cap(-1 / 2,1 / 2)=\varnothing$, the assumption $\beta \leq 1$ implies that also $\operatorname{spec}\left({ }^{Z} Z\right) \cap(-1 / 2,1 / 2)=\varnothing$ in this case.

Now assume that the geometric Witt condition is satisfied. By Theorem 1.1, $\partial$ is essentially self-adjoint. That is, the graph closure of $\partial$ on $C_{c}^{\infty}(M)$ is self-adjoint, with domain $\mathcal{D}$ from Theorem 1.1, and furthermore by the Main Theorem its index satisfies (1.4).

From the Lichnerowicz formula [11],

$$
ð^{*} \partial=\nabla^{*} \nabla+R / 4
$$

where $R$ is the scalar curvature. Thus, for every $\phi \in C_{c}^{\infty}(M),\|ð \phi\|_{L^{2}}=\|\nabla \phi\|_{L^{2}}+\langle R \phi, \phi\rangle_{L^{2}}$. We conclude that for all $\phi \in C_{c}^{\infty}(M)$,

$$
\|ð \phi\|_{L^{2}} \geq\|ð \phi\|_{L^{2}}-\langle R \phi, \phi\rangle_{L^{2}} \geq\|\nabla \phi\|_{L^{2}} \geq 0
$$

This implies in particular that $\mathcal{D}_{\text {min }}(ð)=\mathcal{D} \subset \mathcal{D}_{\min }(\nabla)$, where we recall that $\mathcal{D}_{\text {min }}(P)$ refers to the graph closure of the operator $P$ with domain $C_{c}^{\infty}(M)$. We claim that the index of the operator

$$
\text { ə: } \mathcal{D}^{+} \longrightarrow L^{2}\left(M ; \mathcal{S}^{-}\right)
$$

vanishes, so by formula (1.4), Theorem 1.3(b) holds. In fact, the kernel of $\partial$ on $\mathcal{D}$ consists only of the zero vector, since if $\phi \in \mathcal{D}$ has $\widetilde{\partial} \phi=0$, then since (7.1) holds on $\mathcal{D}, \nabla \phi=0$ also. By the Lichnerowicz formula again, $R \phi=0$, but since by assumption $R$ is not identically zero, $\phi$ must vanish somewhere and by virtue of its being parallel, $\phi \equiv 0$. 


\section{A Appendix}

In this appendix we prove Claim 5.8 by using standard bounds on modified Bessel functions to prove the sup norm bound (5.9): for each $\mu$ with $|\mu|>1 / 2$, and all $|\eta|$,

$$
\left\|\mathcal{N}_{\mu,|\eta|}-\mathcal{N}_{\mu,|\eta|}^{\mathrm{APS}}\right\|<1-\delta .
$$

Among references for modified Bessel functions we recall [6, 9, 10, 57].

To begin with, using the Wronskian equation (3.24), note that

$$
\operatorname{Tr} \mathcal{N}_{\mu, z}=\operatorname{Tr} \mathcal{N}_{\mu, z}^{\mathrm{APS}}=1 .
$$

Thus the difference $\mathcal{N}_{\mu, z}-\mathcal{N}_{\mu, z}^{\text {APS }}$ has two equal eigenvalues and hence its norm is the square root of the determinant. We now assume that $\mu \geq 1 / 2$, since the $\mu \leq-1 / 2$ case is treated the same way. Using (3.24) again, we see that

$$
\begin{aligned}
\operatorname{det}\left(\mathcal{N}_{\mu, z}-\mathcal{N}_{\mu, z}^{\mathrm{APS}}\right) & =-\frac{1}{2}+\frac{1}{2} \frac{z}{\left(\mu^{2}+z^{2}\right)^{1 / 2}}\left(\mu\left(I_{\mu-1 / 2} K_{\mu+1 / 2}-I_{\mu+1 / 2} K_{\mu-1 / 2}\right)\right. \\
& \left.+z\left(I_{\mu+1 / 2} K_{\mu+1 / 2}+I_{\mu-1 / 2} K_{\mu-1 / 2}\right)\right)
\end{aligned}
$$

and we want to show that for some $\delta>0$ independent of $\mu \geq 1 / 2$ and $z \geq 0$,

$$
-1+\delta \leq \operatorname{det}\left(\mathcal{N}_{\mu, z}-\mathcal{N}_{\mu, z}^{\mathrm{APS}}\right) \leq 1-\delta
$$

To begin with, we prove that

$$
0 \leq z I_{\nu}(z) K_{\nu}(z) \leq 1 / 2 \quad \text { for } \quad \nu \geq 1 / 2, z \geq 0 .
$$

In fact, we claim that for $\nu \geq 1 / 2, z K_{\nu}(z) I_{\nu}(z)$ is monotone. To see that this holds, differentiate

$$
\left(z K_{\nu}(z) I_{\nu}(z)\right)^{\prime}=K_{\nu} I_{\nu}+z\left(K_{\nu}^{\prime} I_{\nu}+K_{\nu} I_{\nu}^{\prime}\right)=K_{\nu} I_{\nu}\left(1+\frac{z K_{\nu}^{\prime}(z)}{K_{\nu}(z)}+\frac{z I_{\nu}^{\prime}(z)}{I_{\nu}(z)}\right) .
$$

Thus we want to show that $\left.\frac{z K_{\nu}^{\prime}(z)}{K_{\nu}(z)}+\frac{z I_{\nu}^{\prime}(z)}{I_{\nu}(z)}\right) \geq-1$. Using [10, equation (5.1)], for $\nu \geq 1 / 2$

$$
\left(\frac{z K_{\nu}^{\prime}(z)}{K_{\nu}(z)}\right)^{\prime}+\left(\frac{z I_{\nu}^{\prime}(z)}{I_{\nu}(z)}\right)^{\prime} \leq 0
$$

so the quantity $\frac{z K_{\nu}^{\prime}(z)}{K_{\nu}(z)}+\frac{z I_{\nu}^{\prime}(z)}{I_{\nu}(z)}$ is monotone decreasing. In fact, we claim that

$$
\frac{z K_{\nu}^{\prime}(z)}{K_{\nu}(z)}+\frac{z I_{\nu}^{\prime}(z)}{I_{\nu}(z)} \rightarrow \begin{cases}0 & \text { as } z \rightarrow 0 \\ -1 & \text { as } z \rightarrow \infty\end{cases}
$$

The limit as $z \rightarrow \infty$ can be seen using the large argument asymptotic formulas from [1, Section 9.7], while the limit as $z \rightarrow 0$ follows from the recurrence relations (3.24) and the small argument asymptotics in [1, Section 9.6]. Thus $z K_{\nu}(z) I_{\nu}(z)$ is monotone on the region under consideration. Using the asymptotic formulas again shows that

$$
z K_{\nu}(z) I_{\nu}(z) \rightarrow \begin{cases}0 & \text { as } z \rightarrow 0 \\ 1 / 2 & \text { as } z \rightarrow \infty\end{cases}
$$

so (A.3) holds. 
We can now show the upper bound in (A.2). Using the Wronskian relation in (3.24), we write

$$
\begin{aligned}
\operatorname{det}\left(\mathcal{N}_{\mu, z}-\mathcal{N}_{\mu, z}^{\mathrm{APS}}\right)= & -\frac{1}{2}+\frac{1}{2} \frac{\mu}{\left(\mu^{2}+z^{2}\right)^{1 / 2}}+\frac{1}{2} \frac{z}{\left(\mu^{2}+z^{2}\right)^{1 / 2}}\left(-2 \mu I_{\mu+1 / 2} K_{\mu-1 / 2}\right. \\
& \left.+z\left(I_{\mu+1 / 2} K_{\mu+1 / 2}+I_{\mu-1 / 2} K_{\mu-1 / 2}\right)\right) \\
\leq & \frac{1}{2} \frac{z}{\left(\mu^{2}+z^{2}\right)^{1 / 2}}\left(z\left(I_{\mu+1 / 2} K_{\mu+1 / 2}+I_{\mu-1 / 2} K_{\mu-1 / 2}\right)\right) .
\end{aligned}
$$

Now, if $\mu \geq 1$, by (A.3), the right hand side in the final inequality is bounded by $1 / 2$, establishing the upper bound in (A.1) in this case (with $\delta=1 / 2$ ). If $\mu \in[1 / 2,1]$, we use the following inequalities of Barciz [10, equations (2.3), (2.4)]

$$
\frac{z I_{\nu}^{\prime}(z)}{I_{\nu}(z)}<\sqrt{z^{2}+\nu^{2}} \quad \text { and } \quad \frac{z K_{\nu}^{\prime}(z)}{K_{\nu}(z)}<-\sqrt{z^{2}+\nu^{2}}
$$

for $\nu \geq 0, z \geq 0$. Using these inequalities and the recurrence relation (3.24) gives

$$
\frac{I_{\mu-1 / 2}}{I_{\mu+1 / 2}}<\frac{\sqrt{z^{2}+(\mu-1 / 2)^{2}}+\mu-1 / 2}{z}, \quad \frac{K_{\mu-1 / 2}}{K_{\mu+1 / 2}}<\frac{z}{\sqrt{z^{2}+(\mu+1 / 2)^{2}}+\mu+1 / 2},
$$

so continuing the inequality (A.4) gives

$$
\begin{aligned}
\operatorname{det}\left(\mathcal{N}_{\mu, z}-\mathcal{N}_{\mu, z}^{\mathrm{APS}}\right) \leq & \frac{1}{2} \frac{z}{\left(\mu^{2}+z^{2}\right)^{1 / 2}}\left(z I_{\mu+1 / 2} K_{\mu+1 / 2}\right) \\
& \times\left(1+\frac{\sqrt{z^{2}+(\mu+1 / 2)^{2}}+\mu+1 / 2}{\sqrt{z^{2}+(\mu-1 / 2)^{2}}+\mu-1 / 2}\right) .
\end{aligned}
$$

One checks that for $1 / 2 \leq \mu$, the fraction in the second line is monotone decreasing in $z$, and thus by (A.3), for $z \geq 1$ the determinant is bounded by

$$
\frac{1}{4}\left(1+\frac{\sqrt{1+(\mu+1 / 2)^{2}}+\mu+1 / 2}{\sqrt{1+(\mu-1 / 2)^{2}}+\mu-1 / 2}\right) \leq \frac{1}{4}(1+(1+\sqrt{2})) \leq 1-\delta,
$$

where the middle bound is obtained by checking that the fraction on the left is monotone decreasing in $\mu$ for $\mu \geq 1 / 2$ and equal to $1+\sqrt{2}$ at $\mu=1 / 2$. Thus, we have established the upper bound in (A.2) in the region $z \geq 1$. For $z \leq 1$, rewrite the bound in (A.5) as

$$
\frac{1}{2} \frac{z}{\left(\mu^{2}+z^{2}\right)^{1 / 2}}\left(I_{\mu+1 / 2} K_{\mu+1 / 2}\right) z\left(1+\frac{\sqrt{z^{2}+(\mu+1 / 2)^{2}}+\mu+1 / 2}{\sqrt{z^{2}+(\mu-1 / 2)^{2}}+\mu-1 / 2}\right) .
$$

For $\mu \geq 1 / 2$, by [57], the function $I_{\mu+1 / 2}(z) K_{\mu+1 / 2}(z)$ is monotone decreasing, and by the asymptotic formulas it is goes to $1 / 2$ as $z \rightarrow 0$. Thus in $0 \leq z \leq 1$ the determinant is bounded about by

$$
\frac{1}{4} z\left(1+\frac{\sqrt{z^{2}+(\mu+1 / 2)^{2}}+\mu+1 / 2}{\sqrt{z^{2}+(\mu-1 / 2)^{2}}+\mu-1 / 2}\right) .
$$

This function is monotone increasing in $z$ for $\mu \in[1 / 2,1]$, so the $\max$ is obtained at $z=1$, i.e., it is bounded by the left hand side of (A.6), in particular by $1-\delta$ for the same $\delta$. This establishes the upper bound in (A.2). 
Finally we establish the lower bound. First, we rewrite the determinant again, this time using the Wronskian relation in the opposite direction to obtain

$$
\begin{aligned}
\operatorname{det}\left(\mathcal{N}_{\mu, z}-\mathcal{N}_{\mu, z}^{\mathrm{APS}}\right)= & -\frac{1}{2}-\frac{1}{2} \frac{\mu}{\left(\mu^{2}+z^{2}\right)^{1 / 2}}+\frac{1}{2} \frac{z}{\left(\mu^{2}+z^{2}\right)^{1 / 2}}\left(2 \mu I_{\mu-1 / 2} K_{\mu+1 / 2}\right. \\
& \left.+z\left(I_{\mu+1 / 2} K_{\mu+1 / 2}+I_{\mu-1 / 2} K_{\mu-1 / 2}\right)\right) .
\end{aligned}
$$

Now, recalling that $z I_{\mu+1 / 2}(1) K_{\mu+1 / 2}(1)$ is monotone increasing, using the asymptotic formulas [1, equations $(9.7 .7),(9.7 .8)]$ we see that

$$
I_{\mu+1 / 2}(1) K_{\mu+1 / 2}(1) \rightarrow \frac{1}{2(\mu+1 / 2)}
$$

as $\mu \rightarrow \infty$, we use the inequality [6, equation (11)], namely

$$
I_{\mu-1 / 2}(z) \geq \frac{\mu-1 / 2+\left(z^{2}+(\mu+3 / 2)^{2}\right)^{1 / 2}}{z} I_{\mu+1 / 2}(z) .
$$

On the region $z \in[0,1], \mu-1 / 2+\left(z^{2}+(\mu+3 / 2)^{2}\right)^{1 / 2} \geq \delta_{0}>0$. Dropping the terms with equal order in (A.7) then gives

$$
\begin{aligned}
\operatorname{det}\left(\mathcal{N}_{\mu, z}-\mathcal{N}_{\mu, z}^{\mathrm{APS}}\right) & >-1+\frac{1}{2} \frac{z}{\left(\mu^{2}+z^{2}\right)^{1 / 2}} 2 \mu I_{\mu-1 / 2} K_{\mu+1 / 2} \\
& \geq-1+\frac{1}{2} \frac{2 \mu}{\left(\mu^{2}+z^{2}\right)^{1 / 2}} I_{\mu+1 / 2} K_{\mu+1 / 2}\left(\mu-1 / 2+\left(z^{2}+(\mu+3 / 2)^{2}\right)^{1 / 2}\right) \\
& \geq-1+\delta_{0} \frac{1}{2} \frac{\mu-1 / 2+\left(z^{2}+(\mu+3 / 2)^{2}\right)^{1 / 2}}{\left(\mu^{2}+z^{2}\right)^{1 / 2}} \geq-1+\delta .
\end{aligned}
$$

This completes the proof of (A.2).

\section{Acknowledgements}

P.A. was supported by NSF grant DMS-1104533 and Simons Foundation grant \#317883. The authors are happy to thank Rafe Mazzeo and Richard Melrose for many useful and interesting discussions. They are also grateful to the comments of the anonymous referees, particularly their suggestion of Remark 3.5.

\section{References}

[1] Abramowitz M., Stegun I.A., Handbook of mathematical functions with formulas, graphs, and mathematical tables, National Bureau of Standards Applied Mathematics Series, Vol. 55, U.S. Government Printing Office, Washington, D.C., 1964.

[2] Albin P., Leichtnam É., Mazzeo R., Piazza P., The signature package on Witt spaces, Ann. Sci. Éc. Norm. Supér. (4) 45 (2012), 241-310, arXiv:1112.0989.

[3] Albin P., Leichtnam É., Mazzeo R., Piazza P., Hodge theory on Cheeger spaces, J. Reine Angew. Math., to appear, arXiv:1307.5473.

[4] Ammann B., Humbert E., Morel B., Mass endomorphism and spinorial Yamabe type problems on conformally flat manifolds, Comm. Anal. Geom. 14 (2006), 163-182, math.DG/0503299.

[5] Ammann B., Lauter R., Nistor V., Pseudodifferential operators on manifolds with a Lie structure at infinity, Ann. of Math. 165 (2007), 717-747, math.AP/0304044.

[6] Amos D.E., Computation of modified Bessel functions and their ratios, Math. Comp. 28 (1974), $239-251$.

[7] Atiyah M., Lebrun C., Curvature, cones and characteristic numbers, Math. Proc. Cambridge Philos. Soc. 155 (2013), 13-37, arXiv:1203.6389. 
[8] Atiyah M.F., Patodi V.K., Singer I.M., Spectral asymmetry and Riemannian geometry. I, Math. Proc. Cambridge Philos. Soc. 77 (1975), 43-69.

[9] Baricz Á., Bounds for modified Bessel functions of the first and second kinds, Proc. Edinb. Math. Soc. 53 (2010), 575-599.

[10] Baricz Á., Bounds for Turánians of modified Bessel functions, arXiv:1202.4853.

[11] Berline N., Getzler E., Vergne M., Heat kernels and Dirac operators, Grundlehren Text Editions, SpringerVerlag, Berlin, 2004.

[12] Bismut J.M., Cheeger J., $\eta$-invariants and their adiabatic limits, J. Amer. Math. Soc. 2 (1989), 33-70.

[13] Bismut J.M., Cheeger J., Families index for manifolds with boundary, superconnections, and cones. I. Families of manifolds with boundary and Dirac operators, J. Funct. Anal. 89 (1990), 313-363.

[14] Bismut J.M., Cheeger J., Families index for manifolds with boundary, superconnections and cones. II. The Chern character, J. Funct. Anal. 90 (1990), 306-354.

[15] Bismut J.M., Cheeger J., Remarks on the index theorem for families of Dirac operators on manifolds with boundary, in Differential Geometry, Pitman Monogr. Surveys Pure Appl. Math., Vol. 52, Longman Sci. Tech., Harlow, 1991, 59-83.

[16] Bismut J.M., Freed D.S., The analysis of elliptic families. II. Dirac operators, eta invariants, and the holonomy theorem, Comm. Math. Phys. 107 (1986), 103-163.

[17] Booß-Bavnbek B., Wojciechowski K.P., Elliptic boundary problems for Dirac operators, Mathematics: Theory \& Applications, Birkhäuser Boston, Inc., Boston, MA, 1993.

[18] Bott R., Tu L.W., Differential forms in algebraic topology, Graduate Texts in Mathematics, Vol. 82, SpringerVerlag, New York - Berlin, 1982.

[19] Brüning J., The signature operator on manifolds with a conical singular stratum, Astérisque (2009), 1-44).

[20] Brüning J., Seeley R., An index theorem for first order regular singular operators, Amer. J. Math. 110 (1988), 659-714.

[21] Chan S.W., L-classes on pseudomanifolds with one singular stratum, Proc. Amer. Math. Soc. 125 (1997), 1955-1968.

[22] Cheeger J., On the spectral geometry of spaces with cone-like singularities, Proc. Nat. Acad. Sci. USA 76 (1979), 2103-2106.

[23] Cheeger J., On the Hodge theory of Riemannian pseudomanifolds, in Geometry of the Laplace Operator (Proc. Sympos. Pure Math., Univ. Hawaii, Honolulu, Hawaii, 1979), Proc. Sympos. Pure Math., Vol. 36, Amer. Math. Soc., Providence, R.I., 1980, 91-146.

[24] Cheeger J., Spectral geometry of singular Riemannian spaces, J. Differential Geom. 18 (1983), 575-657.

[25] Chou A.W., The Dirac operator on spaces with conical singularities and positive scalar curvatures, Trans. Amer. Math. Soc. 289 (1985), 1-40.

[26] Chou A.W., Criteria for selfadjointness of the Dirac operator on pseudomanifolds, Proc. Amer. Math. Soc. 106 (1989), 1107-1116.

[27] Dai X., Adiabatic limits, nonmultiplicativity of signature, and Leray spectral sequence, J. Amer. Math. Soc. 4 (1991), 265-321.

[28] Dai X., Wei G., Hitchin-Thorpe inequality for noncompact Einstein 4-manifolds, Adv. Math. 214 (2007), 551-570, math.DG/0612105.

[29] Dai X., Zhang W.P., Circle bundles and the Kreck-Stolz invariant, Trans. Amer. Math. Soc. 347 (1995), 3587-3593.

[30] Debord C., Lescure J.M., Nistor V., Groupoids and an index theorem for conical pseudo-manifolds, J. Reine Angew. Math. 628 (2009), 1-35, math.OA/0609438.

[31] Fedosov B., Schulze B.W., Tarkhanov N., The index of elliptic operators on manifolds with conical points, Selecta Math. (N.S.) 5 (1999), 467-506.

[32] Gil J.B., Loya P.A., Mendoza G.A., A note on the index of cone differential operators, math.AP/0110172.

[33] Gil J.B., Mendoza G.A., Adjoints of elliptic cone operators, Amer. J. Math. 125 (2003), 357-408, math.AP/0108095.

[34] Gilkey P.B., On the index of geometrical operators for Riemannian manifolds with boundary, Adv. Math. 102 (1993), 129-183.

[35] Grubb G., Heat operator trace expansions and index for general Atiyah-Patodi-Singer boundary problems, Comm. Partial Differential Equations 17 (1992), 2031-2077. 
[36] Hausel T., Hunsicker E., Mazzeo R., Hodge cohomology of gravitational instantons, Duke Math. J. 122 (2004), 485-548, math.DG/0207169.

[37] Hörmander L., The analysis of linear partial differential operators. III. Pseudo-differential operators, Classics in Mathematics, Springer, Berlin, 2007.

[38] Krainer T., Mendoza G.A., Boundary value problems for elliptic wedge operators: the first-order case, in Elliptic and Parabolic Equations, Springer Proc. Math. Stat., Vol. 119, Springer, Cham, 2015, 209-232, arXiv:1403.6894.

[39] Kronheimer P.B., Mrowka T.S., Gauge theory for embedded surfaces. I, Topology 32 (1993), 773-826.

[40] Kronheimer P.B., Mrowka T.S., Gauge theory for embedded surfaces. II, Topology 34 (1995), 37-97.

[41] Lawson Jr. H.B., Michelsohn M.L., Spin geometry, Princeton Mathematical Series, Vol. 38, Princeton University Press, Princeton, NJ, 1989.

[42] Leichtnam E., Mazzeo R., Piazza P., The index of Dirac operators on manifolds with fibered boundaries, Bull. Belg. Math. Soc. Simon Stevin 13 (2006), 845-855, math.DG/0609614.

[43] Lesch M., Operators of Fuchs type, conical singularities, and asymptotic methods, Teubner-Texte zur Mathematik, Vol. 136, B.G. Teubner Verlagsgesellschaft mbH, Stuttgart, 1997, dg-ga/9607005.

[44] Lock M.T., Viaclovsky J.A., An index theorem for anti-self-dual orbifold-cone metrics, Adv. Math. 248 (2013), 698-716, arXiv:1209.3243.

[45] Mazzeo R., Elliptic theory of differential edge operators. I, Comm. Partial Differential Equations 16 (1991), $1615-1664$.

[46] Mazzeo R., Melrose R.B., The adiabatic limit, Hodge cohomology and Leray's spectral sequence for a fibration, J. Differential Geom. 31 (1990), 185-213.

[47] Mazzeo R., Vertman B., Analytic torsion on manifolds with edges, Adv. Math. 231 (2012), 1000-1040, arXiv:1103.0448.

[48] Mazzeo R., Vertman B., Elliptic theory of differential edge operators, II: Boundary value problems, Indiana Univ. Math. J. 63 (2014), 1911-1955, arXiv:1307.2266.

[49] Melrose R.B., Pseudodifferential operators, corners and singular limits, in Proceedings of the International Congress of Mathematicians, Vols. I, II (Kyoto, 1990), Math. Soc. Japan, Tokyo, 1991, 217-234.

[50] Melrose R.B., Calculus of conormal distributions on manifolds with corners, Int. Math. Res. Not. 1992 (1992), 51-61.

[51] Melrose R.B., The Atiyah-Patodi-Singer index theorem, Research Notes in Mathematics, Vol. 4, A K Peters, Ltd., Wellesley, MA, 1993.

[52] Melrose R.B., Fibrations, compactifications and algebras of pseudodifferential operators, in Partial Differential Equations and Mathematical Physics (Copenhagen, 1995; Lund, 1995), Progr. Nonlinear Differential Equations Appl., Vol. 21, Birkhäuser Boston, Boston, MA, 1996, 246-261.

[53] Melrose R.B., Differential analysis on manifolds with corners, available at http://www-math.mit.edu/ rbm/ book.html.

[54] Melrose R.B., Introduction to microlocal analysis, available at http://www-math.mit.edu/ rbm/Lecture notes.html.

[55] Melrose R.B., Nistor V., Homology of pseudodifferential operators. I. Manifolds with boundary, functan/9606005.

[56] Nistor V., Analysis on singular spaces: Lie manifolds and operator algebras, J. Geom. Phys. 105 (2016), 75-101, arXiv:1512.06575.

[57] Penfold R., Vanden-Broeck J.M., Grandison S., Monotonicity of some modified Bessel function products, Integral Transforms Spec. Funct. 18 (2007), 139-144.

[58] Roe J., Elliptic operators, topology and asymptotic methods, Pitman Research Notes in Mathematics Series, Vol. 395, 2nd ed., Longman, Harlow, 1998.

[59] Seeley R.T., Complex powers of an elliptic operator, in Singular Integrals (Proc. Sympos. Pure Math., Chicago, Ill., 1966), Amer. Math. Soc., Providence, R.I., 1967, 288-307.

[60] Taylor M.E., Partial differential equations. II. Basic theory, Applied Mathematical Sciences, Vol. 115, 2nd ed., Springer, New York, 2011,.

[61] Taylor M.E., Partial differential equations. II. Qualitative studies of linear equations, Applied Mathematical Sciences, Vol. 116, 2nd ed., Springer, New York, 2011.

[62] Witten E., Global gravitational anomalies, Comm. Math. Phys. 100 (1985), 197-229. 